\title{
KASL clinical practice guidelines for management of chronic hepatitis B
}

\author{
Korean Association for the Study of the Liver (KASL)*
}

Keywords: Chronic hepatitis B; Treatment; KASL guidelines

\section{PREAMBLE}

\section{Background and Aims}

Clinical practice guidelines for the management of chronic hepatitis B (CHB) were originally published in 2004 by the Korean Association for the Study of the Liver (KASL) in order to provide specific medical information regarding CHB that would facilitate treatment of infected patients. Other than an update on treatment of antiviral resistance in 2014, which is a partial revision, the guidelines for the treatment of CHB have been revised entirely three times in 2007, 2011, and 2015. The Asian-Pacific Association for the Study of the Liver (APASL), the European Association for the Study of the Liver (EASL), and the American Association for the Study of Liver

\begin{abstract}
Abbreviations:
AASLD, the American Association for the Study of Liver Diseases; ACLF, acuteon-chronic liver failure; ALP, alkaline phosphatase; ALT, alanine aminotransferase anti-HAV IgG, hepatitis A virus antibody immunoglobulin $\mathrm{G}$; anti-HBC, antibody to hepatitis B core antigen; anti-HBe, antibody to hepatitis B e antigen; anti-HBs, antibody to hepatitis B surface antigen; APASL, the Asian-Pacific Association for the Study of the Liver; APR, Antiretroviral Pregnancy Registry; APRI, aspartate aminotransferase-platelet ratio index; AST, aspartate aminotransferase; AUROCs, areas under the receiver operating characteristic curve; $\mathrm{BCP}$, basal core promoter; besifovir, besifovir dipivoxil maleate; $C B C$, complete blood count; $C c c$, covalently closed circular; CHB, chronic hepatitis $\mathrm{B}$; Cl, confidence interval; CK, creatine kinase; DAA, direct-acting agents; DMARDs, disease-modifying antirheumatic drugs; EASL, the European Association for the Study of the Liver; EBRT, external beam radiation therapy; eGFR, estimated glomerular filtration rate; FDA, Food and Drug Administration; FIB-4, fibrosis-4; GGT, gamma glutamyltranspeptidase; GRADE, Grading of Recommendations, Assessment, Development and Evaluation; HAART, highly active antiretroviral therapy; HAIC, hepatic artery infusion chemotherapy; $\mathrm{HBcAg}$, hepatitis B core antigen; $\mathrm{HBeAg}$, hepatitis $\mathrm{B}$ e antigen; $\mathrm{HBIG}$, hepatitis B immunoglobulin; $\mathrm{HBsAg}$, hepatitis B surface antigen; HBV, hepatitis B virus; HCC, hepatocellular carcinoma; HCV, hepatitis C virus; HDV, hepatitis delta virus; HIV, human immunodeficiency virus; HSCT, hematopoietic stem cell transplant; IBD, inflammatory bowel disease; INR, international normalized ratio; IQR, interquartile range; IU, international unit; KASL, Korean Association for the Study of the Liver; MDR, multidrug resistance; MELD, Model for End-stage Liver Disease; MRE, magnetic resonance elastography; MTCT, mother-to-child transmission; NA, nucleos(t)ide analogues; OR, odds ratio; ORF, open reading frames; PAGE-B, platelets, age, gender, and hepatitis B scores; PC, precore; $P C R$, polymerase chain reaction; peginterferon, pegylated interferon; PT, prothrombin time; qHBsAg, quantitative HBsAg; RA, rheumatoid arthritis; $\mathrm{R}-\mathrm{CHOP}$, rituximab-cyclophosphamide, hydroxydaunorubicin, Oncovin, prednisone; REACH-B, Risk Estimation for Hepatocellular Carcinoma in Chronic Hepatitis B; RFA, radiofrequency ablation; RR, relative risk; TACE, transarterial chemoembolization; TE, transient elastography; tenofovir AF, tenofovir alafenamide fumarate; tenofovir DF, tenofovir disoproxil fumarate; ULN, upper limit of normal
\end{abstract}

\author{
Corresponding author : KASL (Committee Chair: Hyung \\ Joon Yim) \\ Room A1210 MapoTrapalace, 53 Mapo-daero, Mapo-gu, Seoul 04158, \\ Korea \\ Tel: +82-2-703-0051, Fax: +82-2-703-0071 \\ E-mail: kasl@kams.or.kr (or gudwns21@korea.ac.kr)
}

"Clinical Practice Guidelines Committee of KASL for the Management of Chronic Hepatitis B: Hyung Joon Yim (Committee Chair, Korea University College of Medicine), Ji Hoon Kim (Korea University College of Medicine), Jun Yong Park (Yonsei University College of Medicine), Eileen L. Yoon (Inje University College of Medicine), Hana Park (CHA University School of Medicine), Jung Hyun Kwon (College of Medicine, the Catholic University of Korea), Dong Hyun Sinn (Sungkyunkwan University School of Medicine), Sae Hwan Lee (Soonchunhyang University College of Medicine), Jeong-Hoon Lee (Seoul National University College of Medicine), Hyun Woong Lee (Yonsei University College of Medicine) 
Diseases (AASLD) also presented and continued to revise their clinical practice guidelines, and the latest updates were in 2015 , 2017, and 2018. However, since the medical environment in each country is somewhat different depending on race, region, institution, and economic conditions, it is necessary to revise the Korean guidelines to reflect our medical environment and research results.

The clinical practice guidelines committee has begun revising guidelines to reflect the results of Korean and international research published since the revision of the KASL clinical practice guidelines for management of CHB in 2015 and to develop new recommendations. In particular, recent information on newly available antiviral agents has been added, and the goals and the aims of treatment as well as starting and cessation of treatment have been clearly defined. The present guidelines also summarize updates for management of drug resistance, partial virological response, and side effects. In addition, additional data on the topics of epidemiology, prevention, natural history, diagnosis, monitoring, and management of $\mathrm{CHB}$ in specific situations are reflected in this update. Expert opinions were solicited in cases of insufficient data to make definitive conclusions. However, as the guidelines do not represent a standard treatment protocol, clinicians should keep in mind that the best management may vary depending on the individual patient.

\section{Target population}

Patients newly diagnosed with CHB and those previously diagnosed and treated are the primary target population for these guidelines. In addition, the guidelines have been designed to help manage patients with $\mathrm{CHB}$ and those with other special conditions such as hepatocellular carcinoma (HCC), renal dysfunction, metabolic bone disease, immunosuppression, anticancer chemotherapy, liver/non-liver organ transplantation, or co-infections with other viruses such as hepatitis C virus (HCV), human immunodeficiency virus (HIV), or hepatitis delta virus (HDV). Guidelines for pregnant women or those who are preparing for pregnancy, as well as children and adolescents are also presented separately.

\section{Readership}

These guidelines aim to provide useful information and medical guidelines for clinicians responsible for the diagnosis and treatment of patients with CHB in Korea. It is also intended to provide practical and educational information for residents, fellows, and their supervisors.

\section{Information about the committee and funds}

The Committee for the Revision of Clinical Practice Guidelines for CHB 2018, launched in accordance with the initiative of the Board of Directors of the KASL and approved by the council, was composed of ten hepatologists. In addition, specialists representing the Korean Pediatric Society, Korean Society of Infectious Diseases, and Korean Society for Transplantation were invited to participate as external consultants. The cost of revising the guidelines was covered by the KASL.

\section{Collection of evidence}

The committee searched newly published articles related to hepatitis B from PubMed, MEDLINE (up to 2018), and KoreaMed since publication of the 2015 guidelines and systematically reviewed these articles to recommend updated clinical guidelines based on the latest medical data. In addition, we searched abstracts and proceedings of academic conferences in Korea and abroad and collected necessary data. The language of the related literature was limited to articles published in English and Korean, and the search terms included "hepatitis B," "hepatitis B virus (HBV)," "chronic hepatitis." Other keywords covered clinically important topics related to epidemiology, natural history, prevention, diagnosis and initial evaluation, treatment goals and aims, treatment targets and strategies, drugs, monitoring, and antiviral resistance, as well as special situations.

\section{Levels of evidence and grades of recommendation}

The collected data were analyzed through a systematic review, and the levels of evidence were classified by the revised Grading of Recommendations, Assessment, Development and Evaluation (GRADE) system. The levels of evidence were based on the possibility of change in the estimate of clinical effect by further research, and were described as high (A), moderate (B) or low (C). Classification of grades of recommendation were either strong (1) or weak (2), by the GRADE system, according to the level of evidence, generalizability, the clinical effect of the research result, and socioeconomic aspects. Each recommendation is combined with the level of relevant evidence $(A-C)$ and corresponding recommendation grade $(1,2)$ as follows: A1, A2, B1, B2, C1, C2 (Table 1). 


\section{List of the clinical questions}

The committee listed clinical questions related to CHB treatment that were addressed in the main text and the recommendations (Supplementary Material).

\section{Review of the manuscript}

Initial drafts of the revised guidelines were thoroughly reviewed and agreed upon over the course of several committee meetings. An updated manuscript was reviewed at a meeting of the advisory board and opened to a public hearing where KASL members, members of related organizations, and representatives from patient associations attended. After further modification prior to publication, the final manuscript was approved by the Board of Directors of the KASL.

\section{Announcement of the revised guidelines}

The revised CHB guidelines were released on November 24, 2018. The Korean version can be found on the KASL website (http://www.kasl.org).

\section{EPIDEMIOLOGY}

Of the 3.5 billion patients who suffer from CHB worldwide, 600,000 die from related diseases annually. In Korea, the hepati-

Table 1. Grading of Recommendations, Assessment, Development and Evaluation (GRADE)

\begin{tabular}{|c|c|c|c|c|}
\hline & \multicolumn{4}{|c|}{ Criteria } \\
\hline \multicolumn{5}{|l|}{ Quality of evidence } \\
\hline $\operatorname{High}(\mathrm{A})$ & \multicolumn{4}{|c|}{ Further research is unlikely to change confidence in the estimate of the clinical effect. } \\
\hline Moderate (B) & \multicolumn{4}{|c|}{ Further research may change confidence in the estimate of the clinical effect. } \\
\hline Low (C) & \multicolumn{4}{|c|}{ Further research is very likely to impact confidence on the estimate of clinical effect. } \\
\hline \multicolumn{5}{|l|}{ Strength of recommendations } \\
\hline Strong (1) & \multicolumn{4}{|c|}{$\begin{array}{l}\text { Factors influencing the strength of the recommendation included the quality of the evidence, presumed } \\
\text { patient-important outcomes, and cost. }\end{array}$} \\
\hline Weak (2) & \multicolumn{4}{|c|}{$\begin{array}{l}\text { Variability in preferences and values, or more uncertainty. Recommendation is made with less certainty, } \\
\text { higher cost or resource consumption. }\end{array}$} \\
\hline \multicolumn{5}{|c|}{$\begin{array}{l}\text { Of the quality levels of evidence, we excluded "very low quality (D)" evidence from our guidelines for convenience, which was originally included in the GRAD } \\
\text { system and indicates that any estimate of effect is very uncertain. }\end{array}$} \\
\hline Phases* & Serological marker & ALT & HBV DNA & $\begin{array}{l}\text { Histological } \\
\text { activity }^{\dagger}\end{array}$ \\
\hline CHB, immune-tolerant phase & $\begin{array}{l}\operatorname{HBeAg}(+) \\
\text { Anti-HBe }(-)\end{array}$ & Persistently normal & $\begin{array}{l}\text { Very high levels of viral replication } \\
\text { (HBV DNA levels } \geq 10,000,000 \mathrm{IU} / \mathrm{mL} \text { ) }\end{array}$ & None/Minimal \\
\hline $\begin{array}{l}\text { HBeAg-positive } \mathrm{CHB} \text {, } \\
\text { immune-active phase }\end{array}$ & $\begin{array}{l}\text { HBeAg }(+) \\
\text { may develop anti-HBe }\end{array}$ & $\begin{array}{l}\text { Elevated (persistently } \\
\text { or intermittently) }\end{array}$ & $\begin{array}{l}\text { High levels of viral replication } \\
\text { (HBV DNA levels } \geq 20,000 \mathrm{IU} / \mathrm{mL} \text { ) }\end{array}$ & Moderate/Severe \\
\hline $\mathrm{CHB}$, immune-inactive phase & $\begin{array}{l}\operatorname{HBeAg}(-) \\
\text { Anti-HBe }(+)\end{array}$ & Persistently normal & $\begin{array}{l}\text { Low or undetectable HBV DNA } \\
\text { (HBV DNA levels }<2,000 \mathrm{IU} / \mathrm{mL})\end{array}$ & Minimal \\
\hline $\begin{array}{l}\text { HBeAg-negative CHB, } \\
\text { immune-active phase }\end{array}$ & $\begin{array}{l}\operatorname{HBeAg}(-), \\
\text { Anti-HBe (+/-) }\end{array}$ & $\begin{array}{l}\text { Elevated (persistently } \\
\text { or intermittently) }\end{array}$ & $\begin{array}{l}\text { Moderate to high levels of HBV } \\
\text { replication } \\
\text { (HBV DNA levels } \geq 2,000 \mathrm{IU} / \mathrm{mL} \text { ) }\end{array}$ & Moderate/Severe \\
\hline $\begin{array}{l}\text { Resolved CHB, HBsAg loss } \\
\text { phase }\end{array}$ & $\begin{array}{l}\operatorname{HBsAg}(-) \\
\text { Anti-HBC (+) } \\
\text { Anti-HBs (+/-) }\end{array}$ & Normal & Not detected & - \\
\hline
\end{tabular}

ALT, alanine aminotransferase; HBV, hepatitis B virus; $C H B$, chronic hepatitis $B$; $H B e A g$, hepatitis $B$ e antigen; anti-HBe, antibody to hepatitis $B$ e antigen; $\mathrm{HBsAg}$, hepatitis B surface antigen; anti-HBC, antibody to hepatitis B core antigen; anti-HBs, antibody to hepatitis B surface antigen; +, positive; --, negative.

*There can gray zones among the various phases of the natural course.

${ }^{\dagger}$ Fibrosis stages can progress during the natural course of the disease, but may also vary according to the degree of accumulation of liver injury. 
tis B surface antigen ( $\mathrm{HBsAg}$ )-positive rate was high - up to $10 \%$ - in the 1980s. After introduction of HBV vaccinations in 1983, HBsAg-positivity rates dropped significantly to $3 \%$ by 2008. However, the most common etiologies of liver cirrhosis and/or HCC are related to HBV, and CHB remains prevalent even in the late 2010s. ${ }^{2,3}$

\section{NATURAL HISTORY}

CHB is defined as persistence of serum HBsAg for more than 6 months. The natural course consists of five phases: immune-tolerant, hepatitis $\mathrm{B}$ e antigen ( $\mathrm{HBeAg}$ )-positive immune-active, immune-inactive, HBeAg-negative immune-active, and HBsAg loss (Table 2). Duration of these phases varies, sequences of phases are not continuous in patients, and there can be a gray zone in which the features are not compatible with any specific phase. Therefore, assigning phases of infection or making a decision regarding antiviral treatment based on a single alanine aminotransferase (ALT) and HBV DNA is insufficient (Fig. 1). ${ }^{4}$

\section{Immunological features of $\mathrm{CHB}$ during five phases}

$$
\text { CHB, immune-tolerant phase (Immune-tolerant CHB) }
$$

In cases of perinatal infection, the immune-tolerant phase is characterized by HBeAg positivity, very high levels of serum HBV DNA (generally $\geq 10^{7} \mathrm{IU} / \mathrm{mL}$ ), persistently normal levels of ALT, and minimal or no liver necroinflammation. ${ }^{5,6}$ In a follow-up of immune-tolerant CHB patients, serum ALT was elevated in $16 \%$ of cases, and the follow-up fibrosis stage was not different from the initial stage in those who remained in the immune-tolerant phase for five years. ${ }^{5}$ In another study from Taiwan, 5\% of 240 immunetolerant CHB patients progressed to liver cirrhosis and did not develop HCC in 10 years of follow-up. ${ }^{7}$ However, there was a small in vitro study that suggested early hepatocarcinogenesis could be underway even during the immune-tolerant phase, as was evident by a high level of HBV DNA integration and clonal hepatocyte expansion. ${ }^{8}$ Further studies are needed to confirm these issues.

The immune-tolerant phase can last for more than three decades in patients infected with HBV genotype $\mathrm{C}$ due to late $\mathrm{HBeAg}$ seroconversion. Therefore, many female patients infected with this genotype are in the immune-tolerant phase when they are of childbearing age, which can lead to vertical transmission of HBV to a child. ${ }^{9}$

HBeAg-positive CHB, immune-active phase (Immuneactive $\mathrm{HBeAg}$-positive $\mathrm{CHB}$ )

With increasing age, most patients in the immune-tolerant

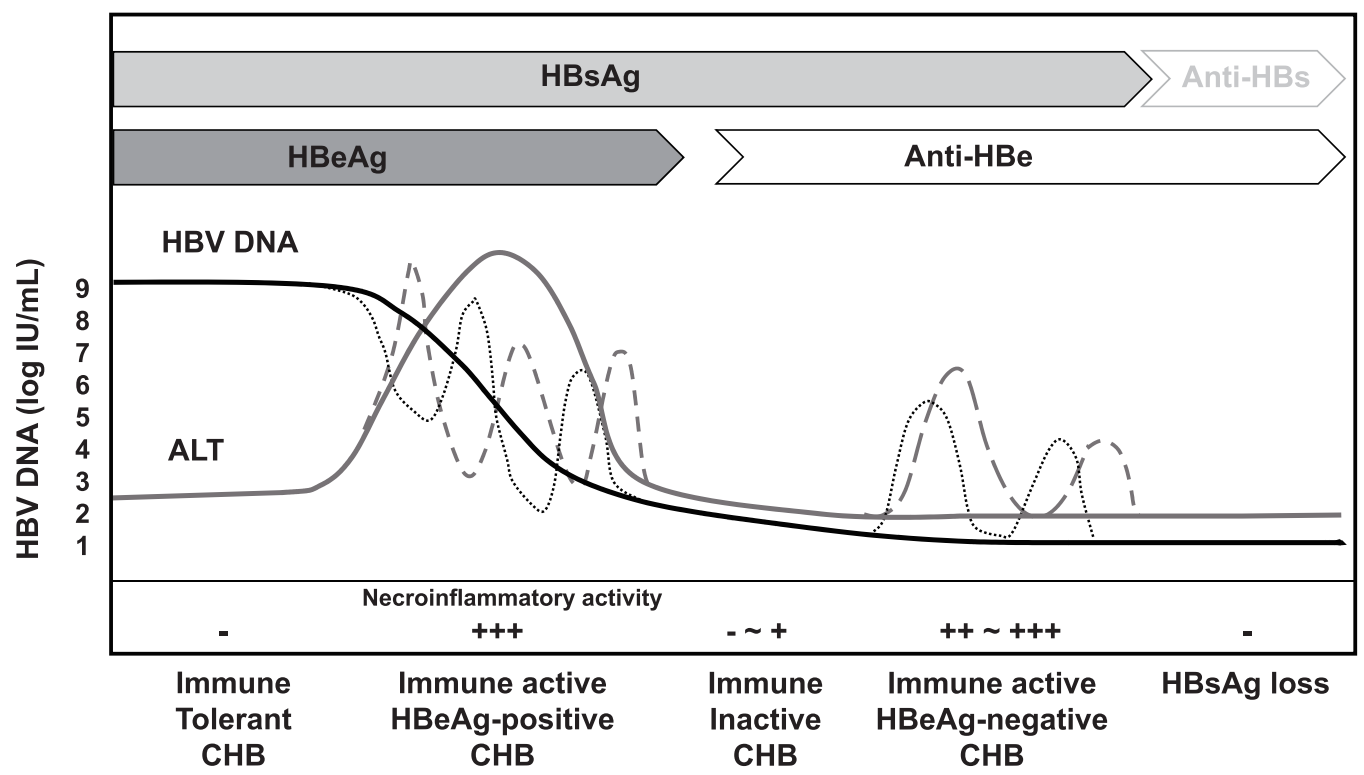

Figure 1. Natural course of chronic hepatitis B. Serological, virological, and biochemical characteristics during five phases of chronic hepatitis B are depicted (modified from Yim et al. ${ }^{17}$ ). Inflammatory activity may vary from minimal (-), mild (+), or moderate (++) to severe (+++). HBsAg, hepatitis B surface antigen; Anti-HBs, antibody to hepatitis B surface antigen; HBeAg, hepatitis B e antigen; Anti-HBe, antibody to hepatitis B e antigen; HBV, hepatitis $B$ virus; ALT, alanine aminotransferase; $C H B$, chronic hepatitis $B$. 
phase experience immune responses to HBV. Such changes are due to increased response of cytotoxic $T$ lymphocytes to hepatitis $\mathrm{B}$ core antigen ( $\mathrm{HBCAg}$ ) and $\mathrm{HBeAg}{ }^{10}$ resulting in destruction of infected hepatocytes. This phase is characterized by HBeAg positivity and fluctuating courses of serum ALT and HBV DNA levels. ${ }^{11,12}$ Histological findings reveal moderate-to-severe necroinflammation. ${ }^{13}$ There can be various stages of liver fibrosis according to the severity of liver injury.

Once HBeAg seroconversion occurs, the natural course of the disease may have one of three clinical features: (1) repeated HBeAg reversion and seroconversion, (2) an immune-inactive phase of CHB, or (3) HBeAg-negative CHB. ${ }^{14,15}$ Typically, $10-40 \%$ of patients who experience seroconversion revert to an $\mathrm{HBeAg}$ positive state and then experience recurrence of seroconversion at least once with progression of hepatitis activity. ${ }^{16,17}$ In particular, reversion frequently occurs in patients with $\mathrm{HBV}$ genotype $\mathrm{C}$, and the rate decreases with age. ${ }^{9}$ Hepatic decompensation, which occurs in $5 \%$ of patients with acute exacerbation, may be fatal. ${ }^{18}$

\section{CHB, immune-inactive phase (Immune-inactive CHB)}

Most patients who seroconvert during the immune-active phase progress to the immune-inactive phase, which is characterized by HBeAg negativity, antibody to hepatitis B e antigen (anti-HBe) positivity, persistent normal ALT levels, and HBV DNA levels below 2,000 $\mathrm{IU} / \mathrm{mL} .^{19-21}$ Typical histological findings in the third phase are mild liver inflammation, ${ }^{19}$ and various stages of liver fibrosis can reflect previous liver injury. ${ }^{22}$

This phase persists for an extended period in most patients, but with a relatively good prognosis. However, an estimated $20 \%$ of such patients will return to the HBeAg-negative or HBeAg-positive immune-active phase, and may experience recurring periods of reactivation and inactivation throughout their lives, which can lead to cirrhosis or HCC. ${ }^{23,24}$

HBeAg-negative $\mathrm{CHB}$, immune-active phase (Immuneactive $\mathrm{HBeAg}$-negative $\mathrm{CHB}$ )

Approximately $20 \%$ of patients who experience HBeAg seroconversion during their immune-active $\mathrm{HBeAg}$-positive phase progress to the immune-active HBeAg-negative phase, with HBV DNA levels $\geq 2,000 \mathrm{IU} / \mathrm{mL}$, increased ALT levels, and active necroinflammation of liver. ${ }^{15}$ These patients show HBeAg negativity because they harbor HBV variants in the precore $(\mathrm{PC})$ or basal core promoter $(B C P)$ regions of HBV DNA, resulting in failure to produce HBeAg. ${ }^{25-27}$ The immune-active HBeAg-negative phase is associated with older age and low rates of prolonged spontaneous disease remission, and most patients in this phase will experience persistent hepatocellular inflammation and progress to hepatic fibrosis and cirrhosis. ${ }^{27-29}$ Severe fluctuations of HBV DNA and ALT levels can make it difficult to differentiate these patients from those in the immune-inactive phase. ${ }^{30}$

\section{HBsAg loss phase (Resolved CHB)}

Patients in the immune-inactive phase subsequently progress to the HBsAg loss or clearance phase at a rate of 1-2\% annually. ${ }^{30-32}$ According to Liaw's prospective data, HBsAg loss occurs in 0.5\% of CHB patients per year, and $0.8 \%$ of asymptomatic chronic HBV carriers per year. ${ }^{33}$ Korean patients reportedly experience a relatively low rate of HBsAg loss $\left(0.4 \%\right.$ annually). ${ }^{34}$ In a few patients, serum HBV DNA can be detected at a very low titer during this phase. HBsAg loss is the state of functional cure, and it is associated with a reduced risk of cirrhosis. However, significant risk of HCC development remains even after HBsAg loss in male patients, and in settings where $\mathrm{HBsAg}$ loss has been achieved late (presence of cirrhosis or age $\geq 50$ years). ${ }^{35,36}$

\section{Risk factors that influence the natural history and progression of liver disease in CHB}

In Korea, the reported annual and five-year accumulated incidences of cirrhosis are $5.1 \%$ and $23 \%$, respectively, while those for HCC are $0.8 \%$ and $3 \%{ }^{37}$ The risk factors for hepatitis B progressing to cirrhosis or HCC can be divided into host, viral, socialenvironmental factors (Table 3). For host factors, cirrhosis, persistent necroinflammation, old age, male gender, family history of HCC, co-infection of other hepatitis virus or HIV affects the risk. ${ }^{17}$ High levels of serum HBV DNA and/or serum HBsAg, HBV genotype $C$, and specific genotypic mutations are included in viral factors. ${ }^{38-47}$ Social-environmental factors for progression to cirrhosis or HCC include alcohol consumption, metabolic syndrome, diabetes, obesity, and smoking. ${ }^{17,46}$ In contrast, coffee, ${ }^{48-50}$ metformin ${ }^{51}$ aspirin, ${ }^{52,53}$ and statins ${ }^{54-59}$ exert protective effects against the development of HCC.

Multiple prognostic prediction models have been developed to estimate the risk of HCC development in CHB patients. The Risk Estimation for Hepatocellular Carcinoma in Chronic Hepatitis B (REACH-B) model, which consists of gender, age, serum ALT, HBeAg, and serum HBV DNA levels, has been developed for HCC risk prediction in non-liver cirrhosis, treatment-naïve $\mathrm{CHB}$ patients. REACH-B model has been validated in Hong Kong and Korean cohort of CHB patients including liver cirrhosis. Areas under 
the receiver operating characteristic curve (AUROCs) for HCC prediction at 3 years, 5 years, and 10 years are $0.77-0.81$ in those cohort. ${ }^{60}$ A modified REACH-B model, which substituted serum HBV DNA for the liver stiffness value from the original REACH-B model, showed better outcomes in assessment of three-year and five-year HCC prediction in several prospective Korean studies. ${ }^{61,62}$ Meanwhile, the PAGE-B (platelets, age, gender, and hepatitis B scores) model, which was developed from Western studies, ${ }^{63}$ has been validated by several Korean retrospective studies. ${ }^{64,65}$ Modified PAGE-B (adding serum albumin) was superior to original PAGE-B in the prediction of five-year HCC risk in Korean CHB patients. ${ }^{65}$

\section{PREVENTION}

The following section describes methods for avoiding new HBV infection in non-infected persons, and for minimizing the risk of disease progression and development of complications in CHB patients.

\section{HBV non-infected persons}

Because chronic HBV infection is endemic in Korea, any person at high risk for liver disease or with suspected liver disease is recommended to have their HBsAg and antibody to hepatitis B surface antigen (anti-HBs) statuses checked. ${ }^{66,67}$ For individuals negative for $\mathrm{HBsAg}$ and anti-HBs $(<10 \mathrm{mlU} / \mathrm{mL})$, and who have not been vaccinated, hepatitis $B$ vaccination is recommended. In particular, 1) patients with chronic liver diseases such as HCV infection, alcohol-related liver disease, fatty liver disease, autoimmune hepatitis, and cirrhosis, as well as those with elevated ALT or as- partate aminotransferase (AST) of unknown etiology ${ }_{1}^{68}$ and 2) patients at increased risk of HBV infection, such as healthcare workers, inmates and staff at correctional facilities, residents and staff of facilities for the developmentally disabled, household members and sexual partners of HBsAg-positive persons, hemodialysis patients, persons who inject drugs, those at risk for sexually transmitted diseases, and HIV-coinfected patients should be vaccinated for hepatitis $B{ }^{67}$

The three doses constituting the hepatitis B vaccine series administered intramuscularly at birth and 1 and 6 months induce a protective antibody response (anti-HBs $>10 \mathrm{mlU} / \mathrm{mL}$ ) in $>90 \%$ of recipients. Most non-responders (44-100\%) subsequently respond to a further three-dose vaccination. ${ }^{69,70}$ Although serological testing for anti-HBs is not necessary after routine vaccination in immunocompetent adults, post-vaccination testing of anti-HBs status is recommended in some subjects, such as newborns of HBV-infected mothers or nine- to 18-month-old infants with family members with $\mathrm{CHB}$, healthcare workers, dialysis patients, workers in dialysis units and operation rooms, immunocompromised subjects (e.g., HIV infected individuals, hematopoietic stem cell transplant (HSCT) recipients, patients undergoing chemotherapy), and sexual partners of patients with chronic HBV infection should be tested 1-2 months after completion of the HBV immunization series. ${ }^{69,70}$ While anti-HBs levels can decline or disappear over several decades, vaccinated subjects remain protected against HBV infection and there is no need for booster vaccinations in immunocompetent individuals. However, an anti-HBs level of $<10 \mathrm{mlU} / \mathrm{mL}$ in dialysis patients indicates an increased risk of HBV infection, and a booster vaccination is needed if annual testing reveals an anti-HBs level of $<10 \mathrm{mIU} / \mathrm{mL}^{.70}$ This also applies to immunocompromised patients. ${ }^{69,70}$

A person without protective anti-HBs exposed to HBV-contami-

Table 3. Factors associated with development of liver cirrhosis and hepatocellular carcinoma in persons with chronic hepatitis B

\begin{tabular}{|c|c|c|c|}
\hline Risk factors & Host & Viral & $\begin{array}{l}\text { Miscellaneous } \\
\text { (social) }\end{array}$ \\
\hline \multirow[t]{5}{*}{ HCC and liver cirrhosis } & Older age ( $>40$ years) & High serum HBV DNA (>2,000 IU/mL) & Alcohol \\
\hline & Persistent ALT elevation & High serum HBsAg & Metabolic syndrome \\
\hline & Male & Genotype C & Diabetes \\
\hline & Concurrent infection (HCV, HDV, HIV) & Delayed HBeAg seroconversion & Obesity \\
\hline & & Basal core promotor mutation & \\
\hline \multirow[t]{2}{*}{$\mathrm{HCC}$} & Presence of cirrhosis & & Aflatoxin \\
\hline & Family history of HCC & & Smoking \\
\hline
\end{tabular}

HCC, hepatocellular carcinoma; HBV DNA, hepatitis B virus deoxyribonucleic acid; ALT, alanine aminotransferase; HBsAg, hepatitis B surface antigen; HCV, hepatitis C virus; HDV, hepatitis D virus; HIV, human immunodeficiency virus; HBeAg, hepatitis B e antigen. 
nated blood or body fluids should receive hepatitis $B$ immunoglobulin (HBIG, $0.06 \mathrm{~mL} / \mathrm{kg}$ ) and the hepatitis $B$ vaccine as soon as possible; preferably within 24 hours, otherwise post-exposure prophylaxis should be initiated within seven days for percutaneous exposure or within 14 days for sexual exposure. ${ }^{71}$ Sexual partners who have not been tested for HBV serological markers, have not completed the full immunization series, or who are negative for anti-HBs should use barrier protection methods, such as condoms.

As HBV is endemic in Korea, the most common etiology of isolated antibody to hepatitis B core antigen (anti-HBC)-positive patients who are negative for HBsAg and anti-HBs is past HBV infection. They rarely require immunization, but those who are at increased risk of HBV infection should be vaccinated for $\mathrm{HBV}^{72,73}$ Isolated anti-HBC positive patients with abnormal liver function results should be considered for the possibility of serum HBV DNA detection

\section{Patients with chronic HBV infection}

Chronically HBV-infected patients are not the indication for HBV vaccination. Co-infection with hepatitis $A$ in HBV carriers increases the risk of mortality 5.6- to 29 -fold. ${ }^{74}$ Therefore, hepatitis A vaccination is recommended for persons negative for the protective hepatitis A virus antibody immunoglobulin $\mathrm{G}$ (anti-HAV $\lg \mathrm{G}) .^{75}$

CHB patients can transmit the virus to others, and should be counseled regarding how to modify their lifestyle to prevent HBV transmission. Mother-to-child transmission (MTCT) is the most important route of HBV infection. Refer to "Pregnant women or women preparing for pregnancy" sections in the "Management in Special Conditions" chapter, for details on antiviral treatment during pregnancy to prevent MTCT. HBIG and vaccination after delivery can prevent $90-95 \%$ of transmission to newborns from HBsAg-positive mothers. ${ }^{76,77}$ Such infants should receive $0.5 \mathrm{~mL}$ HBIG and start the HBV vaccination series within 12 hours of birth.

The rates of HBV infection among newborns from HBsAg-positive mothers were not different between breast- and formulafeeding ( $0-8 \%$ vs. $3-9 \%$, respectively). $)^{78,79}$

Chronic alcohol consumption is an independent risk factor for cirrhosis and HCC, and even more harmful in patients with chronic liver diseases ${ }^{80}$ Abstinence from alcohol is recommended in patients with chronic HBV infection. ${ }^{81}$

According to several retrospective studies, smoking is associated with HCC development, ${ }^{82,83}$ and the risk of HCC development is much higher in smoking CHB patients with metabolic syndrome. $^{84}$

No specific dietary measures have been shown to affect the natural course in CHB patients. However, one prospective study showed fatty liver disease is associated with fibrosis progression independent of viral factors ${ }^{85}$ In addition, patients with metabolic syndrome resulting from diabetes mellitus, hyperlipidemia, and obesity were associated with an increased risk of HCC development in several retrospective studies. ${ }^{86-88} \mathrm{CHB}$ patients should therefore be counseled on lifestyle modifications regarding metabolic syndromes.

\section{[Recommendations]}

1. If $\mathrm{HBsAg}$ and anti-HBs are negative, hepatitis $B$ vaccination is recommended. (A1)

However, vaccination is not necessary if anti-HBc is positive or anti-HBs was lost after past vaccination; nevertheless, vaccination may be recommended in the presence of high risk of HBV infection. (B1)

2. Newborns with HBV-infected mothers should receive HBIG and the hepatitis $B$ vaccine at delivery and complete the recommended vaccination series. (A1)

3. The hepatitis $A$ vaccine should be given to patients with chronic HBV infection who are negative for anti-HAV IgG. (A1)

4. Patients with chronic HBV infection should abstain from alcohol. (A1)

5. Patients with chronic HBV infection are recommended to stop smoking. (B1)

6. Patients with chronic HBV infection are recommended to maintain adequate body weight to prevent metabolic syndrome or fatty liver disease, and to manage metabolic complications including diabetes and hyperlipidemia. (B1)

\section{DIAGNOSIS AND INITIAL EVALUATION}

CHB is defined as the presence of $\mathrm{HBsAg}$ for longer than 6 months. The initial evaluation of CHB patients should include a thorough history and physical examination, with an emphasis on risk factors such as alcohol consumption or drug use, HAV or HCV co-infection, and a family history of chronic HBV infection and HCC. In high-risk groups, the possibility of HDV or HIV co-infection should also be considered. To establish the causal relationship between HBV infection and liver disease, comorbidities such as obesity, diabetes mellitus, and metabolic syndrome should be assessed. Appropriate longitudinal long-term follow-up is crucial for patients with CHB. Serological, virological, and biochemical tests, non-invasive liver stiffness measurement and/or liver biop- 
sies are used to assess HBV replication and the degree of liver injury in patients with CHB.

\section{Antigen/antibody test}

HBsAg immunoassay is a necessary and accurate test for diagnosis of CHB. By definition, patients who remain positive for HBsAg for longer than 6 months are sufficient to diagnose CHB.

Serological tests, including those for anti-HBs and anti-HBC, can assist in screening populations for HBV infection and differentiating among acute, chronic, past infection and immunized individuals.

Persistently positive anti-HBc is shown when an anti-HBs titer from past HBV infection becomes undetectable over time or in cases with occult hepatitis $B$ infection. ${ }^{89-92}$ Patients who recover from HBV infection will be negative for HBsAg and positive for anti-HBs and anti-HBC. Patients who respond adequately to hepatitis $B$ vaccines will be negative on anti- $\mathrm{HBC}$ and positive on antiHBs testing, as anti-HBC emerges only after HBV infection and persists for life.

Laboratory tests for patients with CHB should include those for $\mathrm{HBeAg}$ and anti-HBe. HBeAg positivity generally indicates a high level of viral replication, and anti-HBe positivity a low level. HBeAg-negative, anti-HBe-positive patients with a normal ALT level and an HBV DNA level of $<2,000 \mathrm{IU} / \mathrm{mL}(<10,000$ copies/mL) may be in the inactive phase. HBeAg-negative CHB patients have elevated ALT and an HBV DNA level of $>2,000 \mathrm{IU} / \mathrm{mL}$.

Acute hepatitis A co-infection in CHB patients can result in increased icteric manifestations, longer recovery time, and increased risk of fulminant hepatic failure. Indeed, underlying chronic liver disease is an important risk factor for fulminant hepatic failure and death in patients with acute HAV infection. ${ }^{93-95}$ The seropositivity graph has shifted horizontally to the right for 20 years in age in the last 30 years, and there is a possibility of acute hepatitis A in all age groups. ${ }^{96}$ Therefore, CHB patients should undergo testing for anti-HAV IgG, and all patients with a negative immune status for hepatitis A should receive the HAV vaccine. Laboratory tests should include tests for co-infection with HCV. Additionally anti-HDV, and/or anti-HIV should be tested in those who are at risk. $^{97,98}$

\section{Biochemical tests}

Assessments of the severity of liver disease should include biochemical markers such as AST, ALT, gamma glutamyltranspepti- dase (GGT), alkaline phosphatase (ALP), bilirubin, albumin, and creatinine. A complete blood count (CBC), and prothrombin time should also be assessed. A progressive decline in serum albumin levels and prolongation of the prothrombin time (PT), often accompanied by a decrease in platelet count, are characteristically observed after cirrhosis develops. The serum ALT level has been commonly used in assessments of liver disease and is an important criterion for defining which patients are candidates for therapy. ${ }^{99}$ The ALT level is usually higher than that of AST, but the ratio may be reversed when the disease progresses to cirrhosis. HBVinfected patients with normal or mildly elevated ALT levels have been thought to have no or mild necroinflammation on liver biopsy. However, there is no correlation between the degree of liver cell necrosis and ALT level. ${ }^{100}$

Data from clinical studies have shown that the true normal level of ALT is significantly lower than the previously established limits: $40 \mathrm{IU} / \mathrm{L}$ for males and $30 \mathrm{IU} / \mathrm{L}$ for females. Moreover, data from cohort studies indicate that the upper limit of normal (ULN) ALT and AST levels should be decreased to $30 \mathrm{IU} / \mathrm{L}$ for males and $19 \mathrm{IU} / \mathrm{L}$ for females. ${ }^{100}$ Meanwhile, according to a study in Korea involving 12,000 patients with chronic HBV infection, the best cut-off values for liver-related mortality prediction were $>34 \mathrm{IU} / \mathrm{L}$ in men, and $>30 \mathrm{IU} / \mathrm{L}$ in women. ${ }^{101}$ Despite being a retrospective study, the research included various age groups (40-79 years), did not excluded the data of mild fatty liver-disease patients, and reflected realistic values of Korean patients with chronic HBV infection. Those levels were associated with liver-related mortality prediction, which is the most important issue in clinical settings. Therefore, it would be relevant to use cut-offs of ALT $\leq 34$ IU/L in men, and $\mathrm{ALT} \leq 30 \mathrm{IU} / \mathrm{L}$ in women until this issue can be clarified by further study.

However, ALT activity might also be affected by age, body mass index, gender, abnormal lipid and carbohydrate metabolism, and uremia. ${ }^{100,102}$ Therefore, relying solely on the finding of elevated ALT as a prerequisite for treatment candidacy has limitations.

\section{Serum HBV DNA tests}

Serum HBV DNA testing provides a direct measure of the level of viral replication. This quantification is essential for characterizing the status of infection, diagnosing the disease, making the decision to treat, and subsequent monitoring of patients. It is also important for predicting the risks of cirrhosis and HCC and should be applied to all patients diagnosed with CHB. The most frequently recommended method for serum HBV DNA quantification 
is real-time polymerase chain reaction (PCR). The introduction of the international unit (IU) as a recommended reporting unit for HBV DNA has facilitated standardized reporting and comparison of serum HBV DNA levels; ${ }^{103} 1 \mathrm{IU} / \mathrm{mL}$ is equivalent to roughly 5 copies/mL, but it differs between test equipment types (Roche Diagnostics: 5.8 copies $/ \mathrm{mL}$, Abbott Diagnostics: 3.4 copies $/ \mathrm{mL}$ ). The same test should be utilized for each HBV DNA level test in a given patient in clinical practice to ensure consistency.

\section{HBV genotypes}

HBV genotypes appear to influence the progression of liver disease, risk of HCC, and response to therapy (including interferon therapy). ${ }^{104-106}$ Some studies in Asia have suggested that genotype $C$ is associated more frequently with late $\mathrm{HBeAg}$ seroconversion, $\mathrm{HBV}$ reactivation or $\mathrm{HBeAg}$ seroreversion after achievement of seroconversion, severe liver disease, and HCC than is genotype $B .^{107}$ The specific genotype has also been shown to affect the response to interferon therapy, with the rate of an antiviral response to pegylated interferon (peginterferon) therapy being higher for genotypes $A$ and $B$ than for genotypes $C$ and D. ${ }^{108}$

HBV genotyping can be recommended to help identify patients who might be at greater risk of disease progression and to determine the most appropriate candidates for peginterferon therapy. ${ }^{109}$ However, genotyping is considered unnecessary in Korea, where patients are almost exclusively infected with genotype $C$.

\section{Serum HBsAg quantification}

A quantitative $\mathrm{HBsAg}$ (qHBsAg) assay is used to indirectly assess the amount and transcriptional activity of covalently closed circular (ccc) DNA, which acts as a template for HBV transcription. $\mathrm{HBsAg}$ is not only generated by transcription and translation of cccDNA, but also can be generated from HBV DNA episomally integrated into the host genome. Therefore the role of $q \mathrm{HBsAg}$ as viral replication is more limited than serum HBV DNA. ${ }^{110}$ However, qHBsAg can help differentiate among multiple phases of natural courses, combining HBV DNA levels in the assessment. Serum $q \mathrm{H}-$ $\mathrm{BsAg}$ level is higher in $\mathrm{HBeAg}$-positive patients than in $\mathrm{HBeAg}$ negative patients. In $\mathrm{HBeAg}$-positive patients, qHBsAg level is higher in the immune-tolerant phase than in the immune-active phase. ${ }^{111,112}$ In HBeAg-negative patients, one-time measurement of serum HBV DNA $<2,000 \mathrm{IU} / \mathrm{mL}$ and $\mathrm{HBsAg}<1,000 \mathrm{IU} / \mathrm{mL}$ is suggestive of future inactive carriers. ${ }^{113,114}$ In contrast, among HBeAg-negative patients with lower viral loads (HBV DNA
$<2,000 \mathrm{IU} / \mathrm{mL}), \mathrm{HCC}$ risk is higher in those with a high qHBsAg titer $(>1,000 \mathrm{IU} / \mathrm{mL})$ than in those with a low qHBsAg titer. ${ }^{115}$

Additionally, the role of serum qHBsAg in prediction of ontreatment or off-treatment response has been widely studied. Serum qHBsAg was useful to predict treatment response during peginterferon therapy in $\mathrm{HBeAg}$-positive patients, possibly providing a guide to stopping treatment ealier. ${ }^{116}$ Serum qHBsAg levels were useful predictors of a sustained off-treatment response in CHB patients who were previously treated with nucleos(t)ide analogues (NA). ${ }^{117,118}$

\section{Liver biopsy}

Liver biopsy can be helpful in determining the degree of necroinflammation and stage of fibrosis. Although it is invasive, the rate of serious complications is very low $(1 / 4,000-1 / 10,000)$. A liver biopsy is recommended even in CHB patients with normal ALT levels, to evaluate the need for antiviral treatment in the presence of the risk of significant liver fibrosis, such as increasing age and serum HBV DNA levels. ${ }^{119}$ However, there are limitations in that only a small portion of the liver is sampled, leading to low intra/interobserver reliability. ${ }^{120}$ Also, biopsy may be contraindicated in patients with bleeding tendency. Thus, it is not required when cirrhosis is clinically evident or when treatment is indicated irrespective of the grade of activity or the stage of fibrosis. The efficacy of non-invasive methods such as transient elastography (TE) or serum markers in assessing fibrosis in CHB has increased. ${ }^{120}$

\section{Non-invasive fibrosis tests}

The severity of liver fibrosis and determination of ALT and HBV DNA levels have essential roles in treatment decisions. Non-invasive methods to estimate liver fibrosis have been developed. Commonly used serum markers are aspartate aminotransferase-platelet ratio index (APRI) and fibrosis-4 (FIB-4) index (platelets, ALT, AST, Age). FibroTest, Hepascore, FibroMeter, Enhanced Liver Fibrosis test using direct markers such as serum a-2 macroglobulin, hyaluronic acid, tissue inhibitor of metalloproteinases-1, type III procollagen aminopeptide, apolipoprotein A1, haptoglobin, L-glutamyl transpeptidase are also available. ${ }^{120,121}$

APRI is calculated by the formula of

(AST/ULN for AST) $\times 100 /$ platelet count $\left(\times 10^{9} / \mathrm{L}\right) .^{122}$

APRI was useful for exclusion of significant fibrosis at a low cut- 
off level and diagnosis of cirrhosis at a high cut-off level in several meta-analyses. ${ }^{123,124}$

FIB-4 is calculated by the formula of age $(y r) \times A S T(I U / L) / p l a t e l e t$ count $\left.\left(\times 10^{9} / \mathrm{L}\right) \times \sqrt{\mathrm{ALT}(\mathrm{IU} / \mathrm{L})}\right)^{125}$ According to several studies, FIB-4 is useful for exclusion of significant fibrosis or diagnosis of cirrhosis. $^{126,127}$

TE using Fibroscan ${ }^{\circledR}$ (Echosense, Paris, France) has a high degree of accuracy for assessment of advanced liver fibrosis. It is the most commonly used method for monitoring chronic liver disease because of its non-invasiveness and high-reproducibility. ${ }^{128}$ TE can be performed rapidly (5 min) in outpatient clinics and yields immediate results. ${ }^{129,130}$ However, only procedures involving $\geq 10$ successful measurements are considered reliable. Moreover, a success rate of at least $60 \%$ and an interquartile range (IQR) of less than $30 \%$ of the median value are required (IQR/median). ${ }^{131} \mathrm{TE}$ has limitations in subjects with ascites, obesity, or narrow intercostal spaces. Moreover, the system may yield false-positive results in subjects with acute hepatitis and extrahepatic biliary tract obstruction). ${ }^{132-134}$ In a meta-analysis from Korea, AUROCs for diagnosis of significant fibrosis $(\geq F 2)$ and cirrhosis were 0.86 and 0.93 , respectively, with diagnosis cut-offs for $F 2, F 3$, and F4 of 7.8 $\mathrm{kPa}, 8.8 \mathrm{kPa}, 11.7 \mathrm{kPa}$, respectively. ${ }^{135}$ TE (Fibroscan ${ }^{\circledR}$; Echosense) had greater diagnostic accuracy than APRI or FIB-4 for liver cirrhosis in a study that compared liver biopsy, aspartate aminotransferase-to-alanine aminotransferase ratio, APRI, TE, and FIB-4 in patients with chronic hepatitis. ${ }^{136}$

Newly developed non-invasive tools to assess fibrosis are acoustic radiation force impulse imaging, shear-wave elastography, real-time elastography, and magnetic resonance elastography (MRE), which needs to be further validated in large cohorts of CHB patients. MRE showed high diagnostic accuracy for biopsyconfirmed liver fibrosis in several retrospective studies ${ }^{137,138}$ and is at least as accurate as TE for assessment of fibrosis. ${ }^{139-141}$ MRE was more reliable in the obese patients. ${ }^{142}$

\section{Screening for HCC}

The initial evaluation of patients with CHB should include screening tests for HCC. Periodic surveillance is also needed in these patients to ensure early detection of HCC during follow-up, irrespective of antiviral treatment. Standard tools for HCC surveillance include measuring the alfa-fetoprotein level and ultrasonography every 6 months. ${ }^{143}$ Patients at a high risk of HCC include those older than 40 years and those with cirrhosis even when they are younger than 40 . Periodic surveillance leads to a higher probability for applying curative treatment. ${ }^{144,145}$ Magnetic resonance imaging and computed tomography may be preferred for some patients with severe cirrhosis or obesity, as ultrasonography has poor sensitivity in those conditions. The use of antiviral therapies may lower the risk or delay the progression of disease but cannot prevent all possible complications. Therefore, active surveillance for HCC is required at regular intervals for early diagnosis and treatment.

\section{[Recommendations]}

1. The initial evaluation of patients with $\mathrm{CHB}$ should include taking a detailed medical history and physical examination, with an emphasis on risk factors such as co-infection, alcohol consumption, and family history of HBV infection and HCC. (A1)

2. In the evaluation of CHB patients, CBC, AST, ALT, ALP, GGT, bilirubin, albumin, creatinine, prothrombin time are required. (A1)

3. HBeAg/anti-HBe and serum HBV DNA quantification should be assessed as HBV replication markers in CHB patients. The most frequently recommended method for serum HBV DNA quantification is real-time PCR. (A1)

4. IgG anti-HAV test is recommended in CHB patients. (B1)

5. In patients with $\mathrm{CHB}$, an anti-HCV test is recommended to rule out HCV co-infection. (B1)

6. In patients with CHB, an anti-HDV and an anti-HIV test may be recommended to rule out HDV or HIV co-infection. (B2)

7. Liver biopsy can be performed to determine the degree of liver necroinflammation and fibrosis in CHB patients. (A2)

8. If a liver biopsy is difficult to perform in patients with $C H B$, non-invasive tests such as serum markers or liver elasticity measurement are recommended to assess liver fibrosis. (B1)

9. Patients with $\mathrm{CHB}$ should be tested for HCC regardless of hepatitis B treatment; abdominal ultrasonography and serum alfa-fetoprotein are the surveillance tools that should be performed every 6 months. (A1)

\section{TREATMENT GOAL AND AIMS}

The ultimate goals of hepatitis B treatment are to decrease mortality and increase survival by alleviating hepatic inflammation and preventing the development of fibrosis, which ultimately reduces the frequency of progression of hepatitis to liver cirrhosis or HCC. ${ }^{146-152}$ The ultimate goals could only be achieved by eradication of HBV in the liver in the early stages of infection; however, cccDNA persists in the hepatocyte nucleus despite antiviral treatment until now, so it is difficult to expect complete elimination of HBV. Therefore, it is most important to consistently maintain complete viral suppression. ${ }^{153}$

Since the goals of treatment can only be assessed after a substantially long-term follow-up period, alternative clinical biomark- 
ers reflecting treatment goals may be considered when deciding to discontinue treatment. Currently, clinically available biomarkers that reflect achievement of treatment goals are ALT, HBV DNA, $\mathrm{HBeAg}$, and HBsAg. Thus, ALT normalization, undetectable HBV DNA, HBeAg loss or seroconversion, and HBsAg loss or seroconversion can be used as clinical treatment aims or endpoints. Among these, serum HBsAg loss or seroconversion is the ideal endpoint of CHB treatment. ${ }^{154}$

\section{[Recommendations]}

1. The ultimate goals of $\mathrm{CHB}$ treatment are to decrease mortality from liver disease and improve survival by preventing HBV replication and alleviating hepatic inflammation, and by preventing the progression of fibrosis, development of liver cirrhosis, and HCC. (A1)
2. Clinical endpoints (aims) of treatment are ALT normalization (male $\leq 34 \mathrm{IU} / \mathrm{L}$, female $\leq 30 \mathrm{IU} / \mathrm{L}$ ), undetectable serum HBV DNA, serum $\mathrm{HBeAg}$ loss or seroconversion, and serum $\mathrm{HBsAg}$ loss or seroconversion. Serum HBsAg loss or seroconversion is the ideal endpoint of hepatitis $B$ treatment. (A1)

\section{TREATMENT INDICATION}

Active HBV replication is associated with increased risk of liver damage, progression of liver disease, and liver-related complications. $^{22}$ Nowadays, antiviral therapy has been developed that can effectively inhibit replication of the virus. Inhibition of HBV replication by antiviral therapy can improve hepatic inflammation, normalize serum ALT levels, improve liver fibrosis, reduce the incidence of HCC, and decrease liver-related death. ${ }^{155}$ However,

\section{HBsAg+ > 6 months}

\section{Assess}

- Liver disease severity (include fibrosis assessment)

Clinical phase (include HBeAg/anti-HBe, HBV DNA, AST/ALT)

\begin{tabular}{|c|c|c|c|c|}
\hline \multicolumn{3}{|c|}{ Chronic hepatitis } & $\begin{array}{l}\text { Compensated } \\
\text { cirrhosis } \\
\text { - Treat if serum }\end{array}$ & $\begin{array}{c}\text { Decompen- } \\
\text { sated cirrhosis } \\
\text { - Treat if serum }\end{array}$ \\
\hline $\begin{array}{l}\text { Immune tolerant } \\
\text { - HBeAg+ } \\
\text { - Very high HBV DNA* } \\
\text { - ALT <ULN }{ }^{\dagger} \\
\text { - No significant fibrosis } \\
\text { or inflammation on } \\
\text { non-invasive tests or } \\
\text { liver biopsy }\end{array}$ & $\begin{array}{l}\text { Immune active } \\
\text { - HBeAg+ or HBeAg- } \\
\text { - Elevated HBV DNA } \\
\text { - ALT } \geq 2 \text { XULN or } \\
\text { significant fibrosis or } \\
\text { inflammation on non- } \\
\text { invasive tests or liver } \\
\text { biopsy }\end{array}$ & $\begin{array}{l}\text { Immune inactive } \\
\text { - HBeAg-/anti-HBe+ } \\
\text { - Low-level HBV DNA } \\
\text { (<2,000 IU/mL) } \\
\text { - ALT <ULN } \\
\text { - No significant fibrosis or } \\
\text { inflammation on non- } \\
\text { invasive tests or liver } \\
\text { biopsy }\end{array}$ & $\begin{array}{l}\text { elevated } \quad(\geq 2,000 \\
\mathrm{IU} / \mathrm{mL}) \\
\text { Consider } \\
\text { treatment even if } \\
\text { serum HBV DNA } \\
\text { shows low-level } \\
\text { viremia }(<2,000 \\
\mathrm{IU} / \mathrm{mL})\end{array}$ & $\begin{array}{l}\text { - Consider liver } \\
\text { transplantation }\end{array}$ \\
\hline Monitor & Treat & Monitor & Treat & Treat \\
\hline
\end{tabular}

\section{Grey area: patients who do not fulfill above category}

Close monitoring

- ALT and HBV DNA every 1-3 months, HBeAg/anti-HBe every 2-6 months to see if treatment criteria has developed.

- For patient in persistent grey area, active evaluation with non-invasive assessment of fibrosis (e.g., fibroscan) or liver biopsy can be considered.

- Patients under special population (e.g., pregnancy, patients receiving cancer chemotherapy) may require treatment even if immune tolerant or inactive phase. See section on special population.

- ${ }^{*}$ Serum HBV DNA $\geq 10^{7} \mathrm{IU} / \mathrm{mL} ;{ }^{\dagger}$ An upper limit of normal (ULN) for ALT of $34 \mathrm{IU} / \mathrm{L}$ for males and $30 \mathrm{IU} / \mathrm{L}$ for females; ${ }^{-S e r u m ~ H B V ~ D N A} \geq 20,000 \mathrm{IU} / \mathrm{mL}$ for $\mathrm{HBeAg}$ positive patient and $\mathrm{HBV}$ DNA $\geq 2,000 \mathrm{IU} / \mathrm{mL}$ for $\mathrm{HBeAg}$ negative patient.

Figure 2. Algorithm for management of chronic hepatitis B virus (HBV) infection. Initiation of antiviral therapy should be determined considering severity of liver disease, degree of HBV replication, and presence of liver injury. HBsAg, hepatitis B surface antigen; HBeAg, hepatitis B e antigen; anti-HBe, antibody to hepatitis B e antigen; AST, aspartate aminotransferase; ALT, alanine aminotransferase. 
currently available antiviral therapies cannot eradicate or eliminate the virus. Furthermore, the efficacy and side effects of the same drug may vary depending on the clinical situation. ${ }^{156}$ Therefore benefits and risks of antiviral therapy should be carefully evaluated on an individual basis in the context of the clinical situation. The following three factors are fundamental components that should be taken into consideration when deciding antiviral therapy: 1) The severity of liver disease, 2) the degree of HBV replication, and 3) the presence of liver injury (Fig. 2). The severity of liver disease can be categorized into chronic hepatitis, compensated cirrhosis, and decompensated cirrhosis. The degree of HBV replication can be assessed by measuring serum HBV DNA levels. The presence of liver injury can be estimated using serum ALT levels or can be assessed by a liver biopsy.

\section{CHB, immune-tolerant phase}

The immune-tolerant phase is characterized by $\mathrm{HBeAg}$ positivity, very high serum HBV DNA levels (usually $\geq 10^{7} \mathrm{IU} / \mathrm{mL}$ ), and persistently normal serum ALT levels. In this phase, long-term prognosis is excellent without antiviral therapy. ${ }^{67,157,158}$ To verify the immune-tolerant phase, a liver biopsy is necessary and will show no or mild inflammation without fibrosis on liver biopsy. However, liver biopsy is an invasive procedure with potential complications that limit its widespread use and repetitive testing in clinical practice. Hence, in real-life clinical practice, a combination of clinical findings is typically used to define the immune-tolerant phase without liver biopsy. However, caution should be exercised considering the results of a recent study suggesting that when patients are defined as in the immune-tolerant phase by a combination of clinical findings without liver biopsy (HBeAg positive, high serum HBV DNA levels, normal ALT levels, and no evidence of cirrhosis), HCC and liver cirrhosis-related complications still occur in a considerable number of patients during long-term follow-up. ${ }^{159}$ In several studies, older age, being male, relatively low serum HBV DNA levels, high liver stiffness value, and normal but highnormal ALT levels were factors associated with HCC development or liver-related complications among patients presumed to be in the immune-tolerant phase by combinations of clinical findings without a liver biopsy. ${ }^{159-161}$ The immune-tolerant phase is usually observed in young adults, and is not common in elderly patients. Although other clinical findings suggest the immune-tolerant phase, liver biopsy may show significant fibrosis or necroinflammation in elderly patients, ${ }^{162}$ as age is associated with increased risk of HCC and death during follow-up. ${ }^{159,161}$ Therefore, even when all the other clinical findings suggest the immune-tolerant phase, a liver biopsy can be considered to verify the immune-tolerant phase in older adults. An age cutoff for liver biopsy consideration was suggested to be $30-40$ years; ${ }^{67,97}$ however, evidence to support this approach is limited.

The immune-tolerant phase is also characterized by very high levels of HBV DNA, as there is little or minimal immune response to the virus. ${ }^{67,97}$ In one study, among patients presumed to be in the immune-tolerant phase, relatively low serum HBV DNA level was associated with a higher risk of HCC and death compared to those with very high serum HBV DNA levels $\left(\geq 10^{7} \mathrm{IU} / \mathrm{mL}\right){ }^{159,161}$ Relatively low serum HBV DNA levels indicate that the immune response has already begun to suppress the virus. The immunetolerant phase is also characterized by little or no necroinflammation without liver fibrosis. Hence, significant fibrosis as seen using non-invasive serum fibrosis markers (e.g., APRI, FIB-4) or TE (Fibroscan $^{\circledR}$; Echosense) suggests that patients are not in the genuine immune-tolerant phase.

ALT is a good indicator of liver necroinflammation, so patients in the immune-tolerant phase show persistently normal ALT levels, as there is no or little liver necroinflammation. Hence, patients with slightly elevated ALT levels are more likely to have fibrosis and necroinflammation on a liver biopsy, and have a higher risk of developing complications during follow-up. ${ }^{161,162}$ Therefore, if ALT is at the borderline of ULN or is slightly higher than ULN, this can be a sign that a patient is not genuinely in the immune-tolerant phase. However, careful interpretation is needed in defining normal or elevated ALT levels. There is controversy about what constitutes healthy, normal ALT levels. Elevation of ALT level can be caused by obesity and other conditions not related to HBV. Recently, the cutoff level for ALT associated with increased liver-related mortality among Korean chronic HBV infected patients was reported to be $34 \mathrm{IU} / \mathrm{mL}$ for men and $30 \mathrm{IU} / \mathrm{mL}$ for women. ${ }^{101}$ Therefore, the present guidelines recommend using these values to define normal ALT levels. For patients with the previously mentioned risk factors (older age, relatively low serum HBV DNA levels, non-invasive test suggesting significant fibrosis, or ALT at ULN or slight higher ULN), a liver biopsy can be considered to guide management decisions despite other clinical findings suggesting the patient is in the immune-tolerant phase.

The efficacy of currently available antiviral regimens is limited for patients in the immune-tolerant phase. Long-term treatment may be necessary and treatment discontinuation can be difficult. Antiviral treatment using NAs resulted in a poor antiviral response rate and a low HBeAg seroclearance rate. ${ }^{163}$ Furthermore, when 
NA treatment was discontinued for those who started oral NA therapy at the immune-tolerant phase, all patients showed a rebound of serum HBV DNA levels above 2,000 IU/mL, 70\% showed an elevation of ALT levels, and 55\% had to re-start NA therapy. ${ }^{164}$ However, in one study from Korea that compared 87 NA-treated immune-tolerant CHB patients to 397 monitored immune-tolerant patients as a control group, increased risk of HCC and cirrhosis was observed in the control group despite favorable baseline liver function. ${ }^{165}$ This finding suggests that some patients who are presumed to be in the immune-tolerant phase may develop complications during follow-up, and that antiviral treatment may decrease the risk of developing complication. Further studies are needed to identify appropriate antiviral treatment indications in patients in the immune-tolerant phase.

\section{[Recommendations]}

1. Antiviral therapy is not indicated in CHB patients in the immune-tolerant phase, as defined by $\mathrm{HBeAg}$ positivity, very high serum HBV DNA level $\left(\geq 10^{7} \mathrm{IU} / \mathrm{mL}\right)$, persistently normal ALT level, and no inflammation or fibrosis on liver biopsy. (B1)

2. Liver biopsy can be considered for HBeAg-positive $\mathrm{CHB}$ patients with normal ALT levels to determine antiviral treatment if the patient's age is $\geq 30-40$ years old, serum HBV DNA levels are $<10^{7} \mathrm{IU} / \mathrm{mL}$, non-invasive fibrosis tests suggest significant hepatic fibrosis, or ALT is approaching the borderline of ULN range. (B2)

\section{HBeAg-positive and HBeAg-negative CHB, immune- active phase}

The immune-active phase is characterized by active replication of HBV and moderate or severe necroinflammation with or without fibrosis. A systematic review and meta-analysis of 15 randomized controlled trials and 44 observational studies showed that antiviral treatment in the immune-active phase reduced the risk of cirrhosis, hepatic decompensation, and HCC. ${ }^{155}$ Therefore, patients in the immune-active phase are indicated for antiviral treatment. Nevertheless, careful attention to HCC development is needed, as antiviral treatment cannot completely eliminate the risk of developing $\mathrm{HCC}^{166}$ A recent study from Korea reported a marked reduction in liver disease mortality by widespread use of antiviral treatments against HBV, but paradoxical increased burden of liver cancer. $^{167}$

Active replication of HBV can be confirmed by serum HBV DNA measurement using PCR. Detection of HBV DNA in the serum in- dicates active replication of the virus. However, the lower limit of detection is different among different HBV DNA measurement assays. Moreover, many patients with low-level viremia (serum HBV DNA level <2,000 IU/mL), shows normal ALT levels, and little or no necroinflammation or fibrosis on a liver biopsy, and show favorable outcomes without antiviral therapy. ${ }^{45}$ Hence, not all patients with detectable serum HBV DNA, but patients with serum HBV DNA levels $\geq 2,000-20,000 \mathrm{lU} / \mathrm{mL}$ (10,000-100,000 copies/mL) for HBeAg-positive patients, and serum HBV DNA levels $\geq 2,000 \mathrm{lU} / \mathrm{mL}$ $(10,000$ copies $/ \mathrm{mL})$ for HBeAg-negative patients are considered for antiviral therapy. ${ }^{45,47,168}$

Serum ALT is a convenient indicator of necroinflammation of the liver and can be easily used in clinical practice. ${ }^{169}$ Elevation of ALT suggests hepatocellular injury and requires assessment and evaluation. However, the degree of ALT elevation does not always correlate with necroinflammation of the liver and can be affected by body mass index and gender. ${ }^{100,170}$ ALT elevation can arise from alcohol use, drug use, fatty liver, and other causes unrelated to $H B V,{ }^{170,171}$ and a normal ALT level may not exclude significant liver disease. ${ }^{172}$ Hence, the use of ALT as a criterion for treatment initiation requires consideration of what degree of ALT elevation should be regarded as a threshold to initiate treatment. If the ALT level is elevated more than $\geq 2$ times the ULN, antiviral treatment for HBV is recommended unless the ALT is elevated by other causes. ${ }^{67,97}$ When ALT is elevated above the ULN but $<2$ times the ULN, controversy exists as to whether these patients require antiviral treatment. ${ }^{67,97}$ Patients with serum ALT elevated above the ULN but $<2$ times the ULN have an increased risk of liver cirrhosis and HCC compared to patients with serum ALT within the normal range. ${ }^{173,174}$ Yet, "normal" ALT levels is defined at different cutoff between studies, and "normal" ALT levels also differs by ethnicity. ${ }^{170,175}$ The specific ALT levels used in clinical trials to initiate antiviral therapy also differ. ${ }^{176-181}$ Therefore, sufficient data are not available to judge whether it is necessary to start antiviral treatment in patients with serum ALT elevated above the ULN but $<2$ times the ULN. In this case, trends in serum ALT and HBV DNA levels should be closely monitored to identify possible causes and to verify whether treatment for such patients should be initiated. If a patient shows persistently elevated ALT levels, but those levels remain $<2$ times the ULN, the degree of fibrosis can be further investigated by non-invasive fibrosis tests or by liver biopsy to verify whether patients require antiviral treatment.

Histological assessment of the liver, liver biopsy, is a cornerstone in the evaluation of hepatic necroinflammation and fibrosis. ${ }^{182}$ Findings of moderate to severe necroinflammation or signifi- 
cant fibrosis $(\geq F 2)$ indicate that antiviral treatment for HBV is needed. ${ }^{156}$ However, a liver biopsy is an invasive procedure requiring special resources that limit widespread clinical use. Serum fibrosis biomarkers or TE (Fibroscan ${ }^{\circledR}$; Echosense) of liver are alternatives that can be used to estimate degree of fibrosis. ${ }^{183}$ These non-invasive biomarkers for liver fibrosis are less accurate than liver biopsy. However, they can be used to rule in or rule out patients with significant fibrosis. Recently, treatment initiation based on liver disease severity as assessed by non-invasive tests (e.g., Fibroscan ${ }^{\circledR}\left[\right.$ Echosense]), has been suggested. ${ }^{183}$ However, evidence to support treatment initiation determined by non-invasive tests remains limited at present.

Among HBeAg-positive CHB patients, spontaneous HBeAg seroconversion has been reported for those experiencing increase of ALT level with HBV DNA elevation. ${ }^{184}$ Hence, 3-6 months observation without antiviral treatment can be considered if spontaneous HBeAg seroconversion is expected. ${ }^{184}$ However, biochemical deterioration leading to liver failure is of concern. A prospective cohort study of 90 patients from Korea with HBeAg-positive CHB who were monitored without antiviral therapy showed a very low rate of spontaneous HBeAg seroconversion (1.1\%), while there was frequent biochemical deterioration and one case of liver transplantation due to liver failure. ${ }^{185}$ Therefore, when expecting HBeAg seroconversion, the risk of acute decompensation leading to liver failure warrants careful attention. Another report from Korea showed that spontaneous HBeAg seroconversion can be expected for patients with non-vertical transmission and low serum HBV DNA levels. ${ }^{186}$

CHB patients may present with severe acute exacerbation, characterized by elevated HBV DNA levels, serum ALT levels 5-10 times greater than ULN, jaundice, coagulopathy, ascites, and/or hepatic encephalopathy. They can also be classified as having acute-on-chronic liver failure (ACLF) when they present with symptoms and signs of liver failure. ${ }^{187}$ Severe acute exacerbation can occur spontaneously, ${ }^{188}$ by drug resistant HBV during antiviral therapy, ${ }_{1}^{189}$ by stopping antiviral therapy, ${ }_{1}^{190}$ or by anticancer chemotherapy. ${ }^{191}$ NA therapy reduces mortality in patients with severe reactivation of CHB presenting as ACLF. ${ }^{192}$ Therefore, immediate antiviral treatment is recommended for CHB patients with severe acute exacerbation or ACLF. Some studies have reported a higher mortality rate among entecavir-treated patients than lamivudine-treated patients, ${ }^{193,194}$ but a meta-analysis of three prospective and eight retrospective studies showed similar effects on the mortality rate between entecavir and lamivudine treatment, with a more favorable long-term outcome in entecavir than lami- vudine. ${ }^{187}$ However, antiviral treatment cannot fully prevent progression to liver failure, which may lead to mortality in the case of high Model for End-stage Liver Disease (MELD) score, moderate to severe ascites, and/or aggravation of hepatic encephalopathy ${ }^{195-197}$ Emergent liver transplantation should be considered and prepared. Steroid or plasma exchange has been suggested in cases of severe acute exacerbation and ACLF, but data are currently limited to a small number of cases. ${ }^{198,199}$

Some HBeAg-negative CHB patients show normal or mildly elevated ALT levels despite elevated HBV DNA levels (>2,000 IU/mL). Some patients move to the immune-inactive phase spontaneously -especially patients with low qHBsAg levels and low serum HBV DNA levels. ${ }^{200} \mathrm{HBeAg}$-negative patients are those who have experienced the prior immune-active phase, and there is possibility that various degrees of fibrosis remain in these patients. For those with advanced fibrosis, antiviral treatment can be considered for those with elevated HBV DNA levels regardless of ALT levels..$^{67,97}$ Hence, HBeAg-negative CHB patients showing elevated HBV DNA levels (>2,000 IU/mL) but normal or mildly elevated ALT levels require careful evaluation of their degree of fibrosis to decide if they should undergo antiviral treatment or monitoring.

\section{[Recommendations]}

1. Antiviral therapy is recommended in $\mathrm{HBeAg}$-positive $\mathrm{CHB}$ patients with $\mathrm{HBV}$ DNA $\geq 20,000 \mathrm{IU} / \mathrm{mL}$, or $\mathrm{HBeAg}$-negative CHB patients with HBV DNA $\geq 2,000 \mathrm{IU} / \mathrm{mL}$ if serum ALT level is $\geq 2$ times the ULN. (A1)

In cases where ALT is 1-2 times the ULN, close ALT monitoring or liver biopsy can be considered. Antiviral therapy is recommended if liver biopsy reveals moderate to severe necroinflammation or significant fibrosis ( $\geq F 2$ ). (A1)

Non-invasive fibrosis tests can be used to guide management decisions in cases where a liver biopsy is not feasible. (B1)

2. In patients with $\mathrm{HBeAg}$-positive or $\mathrm{HBeAg}$-negative $\mathrm{CHB}$, prompt antiviral therapy should be initiated in the case of acute exacerbation, with elevation of ALT $\geq 5-10$ times the ULN, signs of liver failure such as jaundice, PT prolongation, ascites, or hepatic encephalopathy. (A1)

3. In HBeAg-negative CHB patients with HBV DNA $\geq 2,000 \mathrm{IU} / \mathrm{mL}$ and normal ALT levels, follow-up can be considered. Otherwise, liver biopsy or non-invasive fibrosis tests can be considered for assessment of the degree of necroinflammation and/or fibrosis in order to determine whether treatment is needed. (B2)

\section{CHB, immune-inactive phase}

The immune-inactive phase is characterized by HBeAg-negative, 
anti-HBe-positive, persistently normal ALT levels, and undetectable or low $(<2,000 \mathrm{IU} / \mathrm{mL})$ serum HBV DNA levels. In this phase, long-term outcome without antiviral treatment is good for those without advanced fibrosis. ${ }^{45}$ In contrast, risk of HCC is not low for patients with advanced fibrosis. ${ }^{201}$ The immune-inactive phase is a dynamic phase that can reactivate to an immune-active phase. ${ }^{15}$ Hence, patients in the immune-inactive phase require careful assessment of the degree of fibrosis and close monitoring of serum ALT and HBV DNA levels to verify whether they remain in the immune-inactive phase.

HBsAg loss or seroclearance is observed in $1-2 \%$ of patients per year in the immune-inactive phase. ${ }^{31,34} \mathrm{HBsAg}$ seroclearance is considered a surrogate endpoint for a functional cure of CHB. Hence, several studies investigated whether antiviral therapy in the immune-inactive phase can further induce HBsAg seroclearance. ${ }^{202}$

Patients who remain in the immune-inactive phase are those with a low risk for $\mathrm{HCC}$ or liver-related complications during follow-up without antiviral treatment. The clinical benefit of inducing HBsAg loss by antiviral treatment in the immune-inactive phase, in terms of achieving treatment goals for $\mathrm{CHB}$ (improving overall survival or preventing the development of $\mathrm{HCC}$ ), has not yet been demonstrated and requires further investigation.

\section{[Recommendations]}

1. Antiviral treatment is not indicated in $\mathrm{CHB}$ patients in the immune-inactive phase, determined by serum HBV DNA $<2,000 \mathrm{IU} / \mathrm{mL}$, a normal ALT level, and no evidence of advanced liver fibrosis. (B1)

\section{Compensated cirrhosis}

Antiviral treatment for compensated cirrhosis patients can decrease the risk of HCC and liver-related complications, ${ }^{155}$ and can improve liver fibrosis. ${ }^{149,203}$ Serum ALT level may not be elevated in patients with cirrhosis, and the risk of developing a complication is high even for those with normal ALT levels. ${ }^{204}$ Hence, cirrhotic patients with active HBV replication require antiviral treatment regardless of ALT levels. For cirrhotic patients, the risk of HCC decreases but remains even after achieving a virological response by antiviral therapy, ${ }^{205}$ requiring HCC surveillance.

For compensated cirrhosis patients, those with elevated HBV DNA levels $(\geq 2,000 \mathrm{IU} / \mathrm{mL})$ are indicated for antiviral therapy. For patients with detectable but low-level viremia $(<2,000 \mathrm{lU} / \mathrm{mL})$, recent EASL and AASLD guidelines recommend antiviral therapy. ${ }^{67,97}$
An observational cohort study from Korea reported that 33\% of compensated cirrhosis patients with low-level viremia experienced HBV DNA elevation $\geq 2,000 \mathrm{IU} / \mathrm{mL}$ during follow-up, and this was associated with an increased risk for developing HCC. ${ }^{206}$ Furthermore, HCC risk was higher for patients who remained at low-level viremia compared to those with undetectable HBV DNA levels, and antiviral treatment was inversely associated with HCC risk in this group. ${ }^{206}$ For compensated cirrhosis patients with low-level viremia, prompt antiviral treatment has the advantage of preventing HBV DNA elevation during follow-up, and may decrease the risk of developing complications in another observational study from Korea. ${ }^{207}$ These data support prompt antiviral therapy for compensated cirrhosis with low-level viremia. However, until now, there have not been any randomized controlled trials that can assess the benefit and risks of prompt antiviral therapy for compensated cirrhosis patients showing low-level viremia.

\section{[Recommendations]}

1. In patients with compensated cirrhosis, antiviral therapy should be initiated regardless of ALT level if serum HBV DNA level is $\geq 2,000 \mathrm{IU} / \mathrm{mL}$. (A1)

2. Antiviral therapy can be considered in compensated cirrhosis patients with detectable but low-level viremia $(<2,000 \mathrm{IU} / \mathrm{mL})$, regardless of ALT level. (B1)

\section{Decompensated cirrhosis}

Decompensated cirrhosis includes cases with ascites, variceal bleeding, hepatic encephalopathy, or jaundice. ${ }^{208}$ Patients with decompensated cirrhosis might be managed in an institution that can respond appropriately to complications, and are candidates for liver transplantation. Antiviral therapy modifies the natural history of decompensated cirrhosis, improves liver function, decreases the need for liver transplantation, and improves survival. ${ }^{151,209}$ However, even if antiviral therapy is administered, it takes time to acquire a virological response and recover clinically. Some patients with severely impaired liver function may not recover despite antiviral therapy, where liver transplantation should be considered for such cases. ${ }^{210}$ Patients with decompensated cirrhosis are prone to liver failure when HBV reactivation occurs, which requires prompt antiviral therapy when serum HBV DNA is detectable, regardless of its serum levels. Administration of interferon is contraindicated because it may cause serious side effects including liver failure even with small doses. ${ }^{211}$ 


\section{[Recommendations]}

1. In patients with decompensated cirrhosis, NAs should be initiated if serum HBV DNA is detected, regardless of ALT level. Liver transplantation should also be considered. (A1)

\section{MONITORING OF PATIENTS WHO ARE NOT INDICATED FOR TREATMENT}

Patients with CHB who are not on antiviral therapy need to be monitored on a regular basis to see if they become indicated for treatment. Patients in the immune-active phase are indicated for antiviral treatment, while those in the immune-tolerant phase and immune-inactive phase are not indicated for antiviral treatment. Serum HBeAg, anti-HBe, AST/ALT, HBV DNA levels, qHBsAg levels, and/or a liver biopsy can be considered to verify whether patients are indicated for antiviral treatment. qHBsAg tests are helpful in differentiating those in the immune-active phase from those in the immune-tolerant or immune-inactive phase. ${ }^{114,212-214}$ Antiviral treatment is considered independent of the natural course of chronic HBV infection in patients with compensated or decompensated cirrhosis. Therefore, the severity of liver disease should be assessed by clinical findings, laboratory results, imaging studies, non-invasive liver fibrosis markers, and/or by performing a liver biopsy.

Chronic HBV infection is a dynamic process that requires regular monitoring. Serum ALT, HBV DNA, and HBeAg/anti-HBe should be monitored on a regular basis, and qHBsAg, non-invasive fibrosis tests, and/or a liver biopsy can be performed additionally during regular monitoring. For those who are not indicated for treatment, ALT and HBV DNA should be monitored at 3-6 months intervals, and $\mathrm{HBeAg} / \mathrm{anti}-\mathrm{HBe}$ monitoring should be performed at 6-12 months intervals. In real-life situations, sometimes it is difficult to categorize patients into those who are indicated for treatment or not (grey area). In such cases, more frequent monitoring of serum ALT and HBV DNA (every 1-3 months) and HBeAg/anti-HBe monitoring (every 2-6 months) can be performed to see if treatment criteria have developed. Despite close monitoring, some patients may remain in the grey area, and for them, non-invasive assessment of liver fibrosis or a liver biopsy should be considered to see whether patients require antiviral treatment and guide further management plans (Fig. 2).

\section{[Recommendations]}

1. In CHB patients not indicated for treatment, monitoring serum ALT and HBV DNA levels every 3-6 months and HBeAg/antiHBe every 6-12 months is recommended to assess if treatment criteria have developed. (B1)

2. If it is uncertain whether treatment is indicated, monitoring serum ALT and HBV DNA levels every 1-3 months and HBeAg/ anti-HBe every 2-6 months are recommended. Otherwise, treatment decisions can be made by non-invasive fibrosis tests or a liver biopsy (B1).

\section{TREATMENT STRATEGY}

Currently approved antiviral treatments include peginterferon alfa and oral NAs. NAs can be classified into drugs with high genetic barriers and drugs with low genetic barriers (Table 4). ${ }^{215}$ When starting antiviral therapy for HBV, peginterferon monotherapy, oral NA monotherapy, or combination therapy with peginterferon plus NA can be considered. ${ }^{216-219}$ Combination treatment with peginterferon plus NA aims to increase the serological response (e.g., HBsAg loss), which cannot be easily achieved by NA alone. ${ }^{216,217}$ However, starting antiviral treatment with peginterferon plus NA offered no significant advantage over peginterferon or NA monotherapy. ${ }^{220,221}$ Hence, in Korea where genotype C HBV infection is prevalent, combination treatment with peginterferon plus NA cannot be recommended as a better initial regimen than peginterferon alone or NA alone treatment.

Peginterferon treatment is recommended for a finite duration and has the advantage of providing immune-mediated control of the HBV and the possibility of achieving a sustained off-treatment response. ${ }^{219}$ However, the major limitation of peginterferon is that it is a parenteral therapy with various side effects and limited efficacy. Peginterferon is also contraindicated in patients with decreased liver function (e.g., decompensated cirrhosis). ${ }^{219}$ Peginterferon treatment can be considered for compensated cirrhosis patients, but risks (possibility of treatment-related side effects and deterioration of liver function) and benefits (immune-mediated control, and sustained off-treatment response) should be carefully considered on an individual basis among highly selected patients. Once treatment with peginterferon has been started, early treatment discontinuation can be considered by monitoring side effects and the virological response during peginterferon treatment.

In contrast, NA treatment has no fixed treatment duration and requires indefinite treatment for most of the cases. ${ }^{156}$ However, 


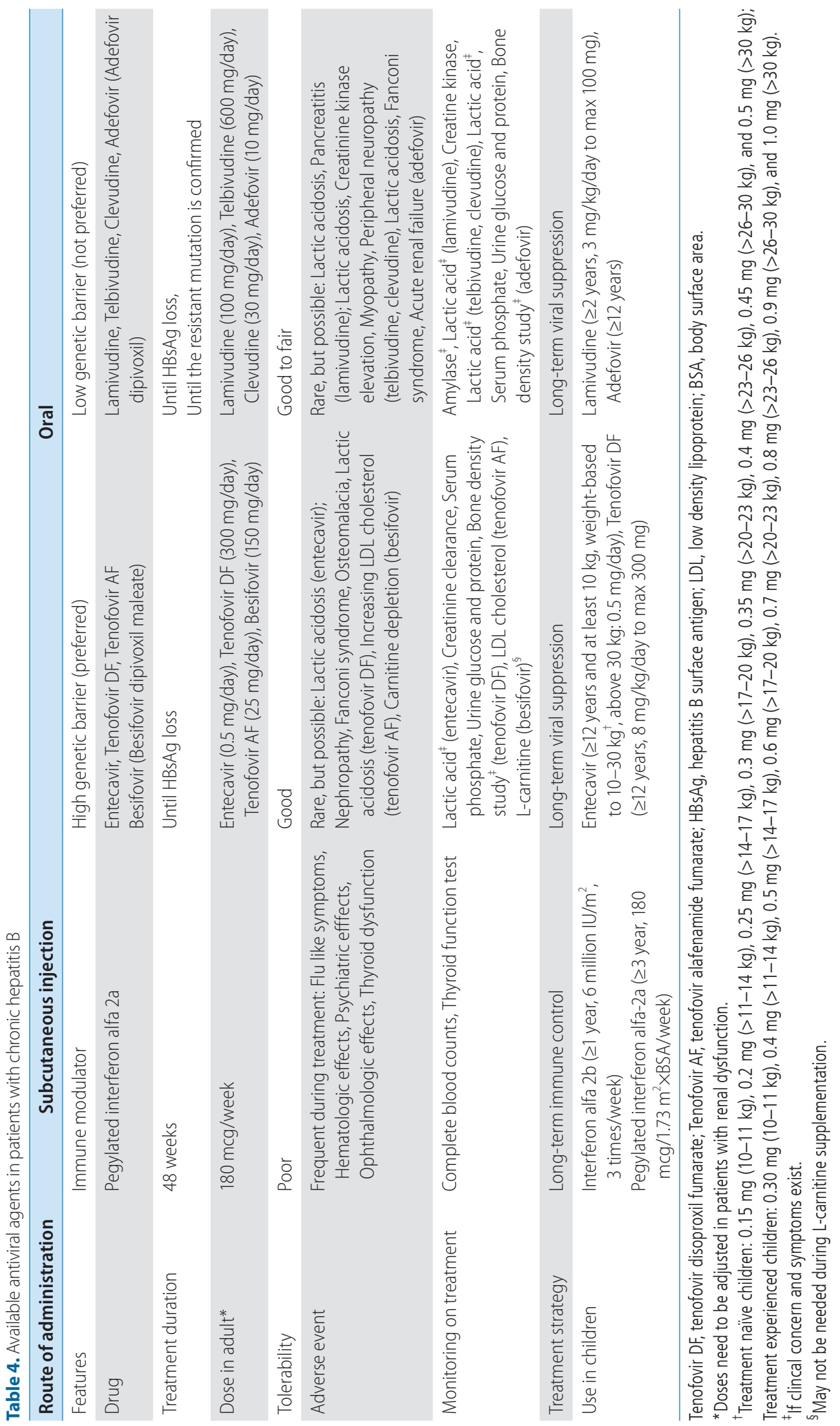


NA treatment has the advantage of being safe in most cases including patients with decompensated cirrhosis. There is a risk of drug resistance with NA treatment, and when drug-resistant HBV mutants develop, it can lead to treatment failure and progression of liver disease. ${ }^{222}$ Newer agents with a high genetic barrier for antiviral resistance have significantly reduced the risk of drug resistance and can effectively suppress HBV replication with monotherapy alone. Hence, when starting antiviral treatment with NAs, monotherapy with a high genetic barrier to resistance is recommended. When choosing a specific NA, one should consider the efficacy and safety of the drug. Although the class effects of NAs remain unclear, each NA has a unique side effect profiles. ${ }^{223}$ Hence, when the efficacy of one NA is expected to be similar to another NA, one should consider patient co-morbidities and the future risk of drug-related side effects when selecting an NA (Refer to "Management in Special Conditions" chapter).

\section{[Recommendations]}

1. For the treatment of patients with $\mathrm{CHB}$, monotherapy using NAs with high genetic barriers to resistance or peginterferon alfa is recommended. (A1)

2. For the treatment of patients with compensated cirrhosis, monotherapy using NAs with high genetic barriers to resistance is recommended. (A1)

If underlying liver function is well preserved, treatment with peginterferon alfa may be considered with careful monitoring for deterioration of liver function and adverse drug reactions. (B2)

3. For the treatment of patients with decompensated cirrhosis, monotherapy using NAs with high genetic barriers to resistance is recommended. (A1)

Peginterferon alfa is contraindicated due to the risk of liver failure. (A1)

\section{THERAPEUTIC AGENTS}

In 2017, tenofovir alafenamide fumarate (tenofovir AF) and besifovir dipivoxil maleate (besifovir) were newly approved for treatment of CHB in adults. Currently, there are eight treatment options for CHB patients in Korea (Table 4).

Among the newly approved drugs, tenofovir AF is a nucleotide analogue with the same mechanism as the existing tenofovir disoproxil fumarate (tenofovir DF) and is maintained at a stable concentration in plasma, effectively metabolized in hepatocytes, and shows similar antiviral activity to tenofovir DF even at a smaller dose. As the amount of systemic exposure is small, tenofovir AF induces less renal and bone toxicity than tenofovir DF. ${ }^{224-227}$

Besifovir is an acyclic nucleotide phosphonate that was developed in Korea as an oral antiviral agent and is similar to adefovir and tenofovir DF in structure. ${ }^{228,229}$ Although clinical data are limited, besifovir has shown little effect on renal and bone toxicity and has similar effects to tenofovir DF in the Phase 3 trial. ${ }^{230}$ Table 4 summarizes newly added drugs and existing treatments including peginterferon alfa 2a. NAs are classified into those associated with high genetic barrier to resistance (entecavir, tenofovir DF, tenofovir AF, besifovir) and those with low genetic barrier to resistance (lamivudine, telbivudine, clevudine, adefovir) (Table 4). In addition, although the efficacy of antiviral agents was not analyzed in head-to-head comparisons, the antiviral efficacy of individual drugs is described in Table 5.

\section{NAs with high genetic barrier}

Entecavir, tenofovir DF, tenofovir AF, and besifovir are recommended as first-line treatment for $\mathrm{HBeAg-positive} \mathrm{and} \mathrm{-negative}$ CHB patients. In particular, many clinical data of entecavir and tenofovir DF have been verified to show their long-term safety and efficacy. ${ }^{231-233}$ Recently, clinical studies with up to 2 years of follow-up have suggested that tenofovir AF and besifovir exhibit better safety profiles than tenofovir DF, with similar antiviral efficacy. ${ }^{224-227,230}$ Further clinical investigation focusing on long-term treatment outcomes should be performed to verify the antiviral efficacy and safety of these new antiviral agents.

\section{NAs with low genetic barrier}

Lamivudine, telbivudine, clevudine, and adefovir are not recommended as first-line treatment for patients with $\mathrm{HBeAg}$-positive or -negative CHB because of viral resistance. However, these drugs have been used in clinical practice before introduction of antiviral agents with high genetic barriers, and they are still being prescribed in patients showing optimal virological responses.

\section{Interferons}

Interferon is a cytokine produced and secreted by immune cells in viral infection and has an antiviral effect and immunity-controlling activity. Although the precise mechanism is unclear, interferon alfa plays a role in destruction of cccDNA and viral mRNA, inhibition of the replication of viral DNA, and effective control of the immune response to virus-infected hepatocytes. ${ }^{234}$ 
Peginterferon is a combination of interferon and polyethylene glycol molecules that has a long half-life, is easier to administer once per week, and has a stronger therapeutic effect compared to conventional interferon. The greatest advantage of peginterferon is the finite treatment period. The rate of HBsAg seroclearance was shown to be $2-7 \%$ at the first year after the end of treatment and increased to $12 \%$ at the fifth year. ${ }^{216,217,220,235-238}$

\section{[Recommendations]}

1. NAs with high genetic barriers to resistance include entecavir, tenofovir DF, tenofovir AF, and besifovir. (A1)

\section{DEFINITION AND PREDICTORS OF ANTIVI- RAL TREATMENT RESPONSE}

\section{Definition of response}

\section{NAs}

The virological response is defined as undetectable HBV DNA by a sensitive PCR assay (Table 6). A maintained virological response is defined by achieving a virological response and maintaining undetectable HBV DNA levels as assessed using a sensitive PCR assay. A partial virological response is defined as a decrease but detectable HBV DNA level after at least 24 weeks of therapy when using low genetic barrier drugs (e.g., lamivudine, telbivudine), and at least 48 weeks of therapy when using high genetic barrier drugs (e.g., entecavir or tenofovir) in compliant patients. A serological response is defined for HBeAg loss and $\mathrm{HBeAg}$ seroconversion for an $\mathrm{HBeAg}$ serological response in HBeAg-positive patients, and HBsAg loss or seroconversion for an HBsAg serological response. A viral breakthrough is defined as an increase in serum HBV DNA level of more than $1 \log _{10} \mathrm{IU} / \mathrm{mL}$ compared with the lowest HBV DNA level on-therapy, or redetection of serum HBV DNA at levels of 10-fold the lower detection limit after achieving a virological response. A virological breakthrough usually precedes a biochemical breakthrough. A biochemical response is defined as a normalization of ALT levels, and a biochemical breakthrough is defined by an increase in ALT levels for patients who have achieved a biochemical response. Genotypic resistance is defined when HBV DNA mutations known to confer

Table 5. Two-year treatment efficacy of pegylated interferon and nucleos(t)ide analogues with high genetic barriers

\begin{tabular}{|c|c|c|c|c|c|}
\hline & Pegylated interferon alfa* & Entecavir $^{\dagger}$ & Tenofovir $\mathrm{DF}^{\dagger}$ & Tenofovir $\mathrm{AF}^{\dagger}$ & Besifovir $^{\dagger}$ \\
\hline \multicolumn{6}{|l|}{ HBeAg positive } \\
\hline Undetectable HBV DNA (\%) & 6-14 (<80 IU/mL) & $70-80(<60 \mathrm{lU} / \mathrm{mL})$ & $75(<29 \mathrm{IU} / \mathrm{mL})$ & $73(<29 \mathrm{IU} / \mathrm{mL})$ & 64-81 (<69 IU/mL) \\
\hline HBeAg loss (\%) & $34-39$ & 39 & 18 & 22 & $14-21$ \\
\hline HBeAg seroconversion (\%) & $29-36$ & $31-33$ & 12 & 18 & $8-21$ \\
\hline ALT normalization (\%) $)^{\ddagger}$ & $32-52$ & $82-87$ & 68 & 75 & $64-79$ \\
\hline HBsAg loss (\%) & $2-7$ & $4-5$ & 1 & 1 & 0 \\
\hline HBsAg seroconversion (\%) & $2-5$ & 2 & 0 & 1 & 0 \\
\hline References & $216,220,236,238$ & 176,319 & 224 & 224 & 334 \\
\hline \multicolumn{6}{|l|}{ HBeAg negative } \\
\hline Undetectable HBV DNA (\%) & $19(<80 \mathrm{IU} / \mathrm{mL})$ & $91-95(<60 \mathrm{IU} / \mathrm{mL})$ & $91(<29 \mathrm{IU} / \mathrm{mL})$ & $90(<29 \mid \mathrm{IU} / \mathrm{mL})$ & $97(<60 \mathrm{IU} / \mathrm{mL})$ \\
\hline ALT normalization $(\%)^{\ddagger}$ & 59 & $78-88$ & 71 & 81 & 88 \\
\hline HBsAg loss (\%) & $4-5$ & $0-1$ & 0 & $<1$ & 0 \\
\hline HBsAg seroconversion (\%) & 3 & 0 & 0 & $<1$ & 0 \\
\hline References & 217 & 179,319 & 224 & 224 & 334 \\
\hline
\end{tabular}

Tenofovir DF, tenofovir disoproxil fumarate; Tenofovir AF, tenofovir alafenamide fumarate; HBeAg, hepatitis B e antigen; HBV, hepatitis B virus; ALT, alanine aminotransferase; $\mathrm{HBsAg}$, hepatitis B surface antigen.

${ }^{*} 6$ months after 1 year treatment.

${ }^{\dagger} 2$ years of continuous treatment.

${ }^{\ddagger}$ ALT normalization was defined by $\leq 35 \mathrm{U} / \mathrm{L}$ (males), and $\leq 25 \mathrm{U} / \mathrm{L}$ (females) for pegylated interferon alfa, and entecavir. For tenofovir DF, and tenofovir AF, it was defined by $\leq 34 \mathrm{U} / \mathrm{L}$ (female $<69$ years) or $\leq 32 \mathrm{U} / \mathrm{L}$ (female $\geq 69$ years) and $\leq 43 \mathrm{U} / \mathrm{L}$ (male $<69$ years) or $\leq 35 \mathrm{U} / \mathrm{L}$ (male $\geq 69$ years). For besifovir, it was defined by $\leq 41 \mathrm{U} / \mathrm{L}$ (males), and $\leq 33 \mathrm{U} / \mathrm{L}$ (females). 
antiviral resistance during antiviral therapy have been detected. Phenotypic resistance is defined as decreased susceptibility (in vitro testing) to inhibition by antiviral drugs associated with genotypic resistance. Cross-resistance is defined as an HBV mutation selected by one antiviral agent that also confers resistance to other antiviral agents. HBV resistance to NAs is characterized by the presence of HBV variants with amino-acid substitutions that confer reduced susceptibility to the administered NA. Such resistance may result in primary treatment failure or virological breakthrough during therapy.

\section{Peginterferon alfa}

A primary non-response to peginterferon alfa is defined as a decrease of less than $1 \log _{10} \mathrm{IU} / \mathrm{mL}$ in serum HBV DNA from baseline to after 3 months of therapy. A virological response is defined as an HBV DNA level of less than 2,000 IU/mL after 6 months or at the end of therapy. A sustained off-therapy virological response is defined as an HBV DNA level of less than 2,000 IU/mL at least 6 months after the end of therapy. A serological response is defined by HBeAg loss or HBeAg seroconversion for an HBeAg serological response in patients with $\mathrm{HBeAg}$-positive $\mathrm{CHB}$, and $\mathrm{HBs} \mathrm{Ag}$ loss or HBsAg seroconversion for HBsAg serological response.

\section{Predictors of response}

\section{NAs}

Pre-treatment serum ALT levels, HBV DNA levels, HBeAg levels, and qHBsAg levels are factors associated with the virological response. ${ }^{99,239}$ Serum HBV DNA levels, ALT levels, severe necroinflammation as observed on a liver biopsy, and a maintained virological response are factors associated with the HBeAg serological response in $\mathrm{HBeAg}$-positive $\mathrm{CHB} .{ }^{240-243}$ When using low genetic barrier drugs such as lamivudine, adefovir, or telbivudine, undetectable HBV DNA at 6-12 months of treatment was also associ-

Table 6. Definition of response to antiviral therapy for chronic hepatitis B

\begin{tabular}{|c|c|}
\hline Category of response & Definition \\
\hline \multicolumn{2}{|l|}{ Nucleos(t)ide analogues } \\
\hline Virological response & Decrease in serum HBV DNA to an undetectable level on real-time PCR assay. \\
\hline Partial virological response & $\begin{array}{l}\text { Decrease in serum HBV DNA of more than } 2 \log _{10} \mathrm{IU} / \mathrm{mL} \text { but detectable HBV DNA on real-time } \\
\text { PCR assay after at least } 12 \text { months of therapy with high-potency NAs, or after at least } 6 \text { months } \\
\text { of therapy with low-potency NAs in compliant patients. }\end{array}$ \\
\hline Virological breakthrough & $\begin{array}{l}\text { Increase in serum HBV DNA of more than } 1 \log _{10} \mathrm{IU} / \mathrm{mL} \text { compared to the lowest value, or } \\
\text { redetection of serum HBV DNA at levels } 10 \text {-fold the lower detection limit after achieving a } \\
\text { virological response. }\end{array}$ \\
\hline Serological response (HBeAg) & HBeAg loss or HBeAg seroconversion \\
\hline Serological response (HBsAg) & HBsAg loss or HBsAg seroconversion \\
\hline Biochemical response & Normalization of ALT level \\
\hline Biochemical breakthrough & Increase in serum ALT level after ALT normalization on antiviral therapy. \\
\hline Genotypic resistance & Detection of HBV mutations known to confer antiviral resistance during antiviral therapy. \\
\hline Phenotypic resistance & $\begin{array}{l}\text { Decreased susceptibility (in vitro testing) to inhibition by antiviral drugs associated with } \\
\text { genotypic resistance. }\end{array}$ \\
\hline Cross resistance & $\begin{array}{l}\text { HBV mutation selected by one antiviral agent that also confers resistance to other antiviral } \\
\text { agents. }\end{array}$ \\
\hline \multicolumn{2}{|l|}{ Pegylated interferon alfa } \\
\hline Primary non-response & Decrease in serum HBV DNA $<1 \log _{10} 1 \mathrm{U} / \mathrm{mL}$ after 3 months of peg-interferon alfa therapy. \\
\hline Virological response & Decrease in serum HBV DNA of less than 2,000 IU/mL after 6 months and at the end of therapy. \\
\hline Sustained off therapy virological response & Serum HBV DNA levels <2,000 IU/mL for at least 6 months after the end of therapy. \\
\hline Serological response (HBeAg) & HBeAg loss or HBeAg seroconversion \\
\hline Serological response (HBsAg) & HBsAg loss or HBsAg seroconversion \\
\hline
\end{tabular}

$H B V$, hepatitis B virus; PCR, polymerase chain reaction; NAs, nucleos(t)ide analogues; HBeAg, hepatitis B e antigen; HBsAg, hepatitis B surface antigen; ALT, alanine aminotransferase. 
ated with a virological response. ${ }^{244-247}$ Caucasian patients, those infected with HBV genotype A or D, males (as opposed to females), and the virological response were factors associated with HBsAg serological response during entecavir therapy. ${ }^{248}$ Caucasian race, less than 4 years of infection, HBV genotype $A$ or $D$, and a reduction in HBsAg levels $>1 \log _{10} \mathrm{U} / \mathrm{mL}$ by week 24 were factors associated with HBsAg serological response during tenofovir therapy. ${ }^{249}$ In Asian patients with CHB, achieving a viral suppression took longer for patients who had a high baseline viral load ( $\geq 9 \log _{10}$ copies $/ \mathrm{mL}$ ). ${ }^{250}$ HBV genotype was not associated with the virological response to NA therapy.

\section{Peginterferon alfa}

The HBV genotype is associated with the treatment response to peginterferon alfa therapy. Those with HBV genotype A or B showed a more favorable HBeAg response, HBsAg response, and virological response than those with HBV genotype $\mathrm{C}$ or D. ${ }^{108,220,251,252}$ In Korea, almost all patients are infected with HBV genotype C, which should be considered when treating patients with peginterferon. High serum ALT levels, low HBV DNA levels, severe necroinflammation, and HBV genotype are factors associated with $\mathrm{HBeAg}$ serological response in HBeAg-positive CHB. ${ }^{216,253}$ High serum ALT levels, low HBV DNA levels, young age, and female sex are factors associated with the virological response in HBeAgnegative CHB. ${ }^{217,253}$ On-treatment factors, such as HBV DNA levels, quantitative HBeAg levels, and qHBsAg levels, are also associated with virological response during peginterferon therapy. 116,25-256 $^{2}$

\section{MONITORING DURING ANTIVIRAL TREAT- MENT}

\section{NAs}

Persistent HBV replication during antiviral treatment is a major risk for hepatitis progression and viral mutation. ${ }^{257}$ Serum HBV DNA should be measured every 1 to 6 months during antiviral therapy to facilitate treatment adjustments based on serum HBV DNA levels.

Although serum HBV DNA is less than 2,000 IU/mL during therapy, the incidence of HCC is higher in patients with detectable HBV DNA persistently or intermittently than in patients with undetectable HBV DNA persistently. ${ }^{205}$ Therefore, serum HBV DNA should be measured every 3 to 6 months during antiviral therapy even after virological response. Serum HBV DNA reduction to an undetectable levels by real-time PCR $(<10-15 \mathrm{IU} / \mathrm{mL})$ should ideally be achieved. ${ }^{97,258}$

Although qHBsAg levels is less likely to decrease with NAs compared to peginterferon alfa, ${ }^{259-261}$ the degree of reduction in HBV DNA is correlated with the degree of reduction in HBsAg levels. ${ }^{259}$ Low pretreatment HBsAg levels and greater HBsAg decline after 24 weeks of treatment were reported to be positive predictors of a long term virological response. ${ }^{262-264}$ In patients with CHB having received ten years of NA therapy, low baseline HBsAg levels $(<1,000 \mathrm{IU} / \mathrm{mL})$ and a greater rate of $\mathrm{HBsAg}$ reduction on-therapy (>0.166 $\log _{10} \mathrm{IU} / \mathrm{mL} /$ year) were predictive of HBsAg loss. ${ }^{265}$ Low HBsAg levels (10-200 IU/mL) on cessation of therapy have been reported to be a good predictor of persistent virological response and HBsAg loss after antiviral cessation. ${ }^{118,266-269}$ Therefore, monitoring of qHBsAg could be helpful in practice.

Drug compliance and emergence of antiviral-resistance mutations should be monitored in patients who develop virological breakthrough while receiving NAs, and an appropriate rescue therapy should be initiated if necessary (Fig. 3). ${ }^{35,79,270-272}$

Most NAs are excreted through the kidney, and hence dose adjustment is required in patients with renal insufficiency (refer to section on renal impairment). Regular monitoring of renal function and bone mineral density should be performed in patients receiving adefovir or tenofovir DF. ${ }^{27,274} \mathrm{~A}$ large prospective study of entecavir-related carcinogenicity found comparable cancer incidence between entecavir and other NAs. ${ }^{232}$ There have been few reports on telbivudine-related myositis; however, monitoring of serum creatine kinase (CK) levels is recommended due to the possibility of CK elevation. ${ }^{178,275}$ Levels Serum CK levels and related symptoms should also be monitored in patients receiving clevudine (Fig. 3). ${ }^{276,277}$

\section{Peginterferon alfa}

The serum CBC and ALT levels of patients receiving peginterferon alfa should be tested monthly. Serum HBV DNA should be measured after 1-3 months of treatment to facilitate treatment adjustments based on serum HBV DNA levels. There is a high probability of HBsAg loss if serum HBV DNA becomes undetectable during treatment. Patients with who are $\mathrm{HBeAg}$-positive should be tested for $\mathrm{HBeAg}$ and anti-HBe at 6 and 12 months during treatment and 6 months post treatment. Patients should be monitored for 6-12 months after treatment cessation. For response prediction, a qHBsAg levels can be used before treatment and after 12 and 24 weeks of treatment. ${ }^{116,255,256,278,279}$ All patients 
treated with peginterferon alfa should be assessed for known adverse effects of interferon at every visit.

\section{[Recommendations]}

1. During treatment with NAs, liver function tests and serum HBV DNA measurement at 1-6 month intervals and HBeAg/anti$\mathrm{HBe}$ at 3-6 month intervals are recommended. (B1) $\mathrm{HBsAg}$ quantification may be considered, which may help predict antiviral response and determine treatment cessation. (B2)

2. During peginterferon alfa therapy, $\mathrm{CBC}$ and liver function tests every month, serum HBV DNA at intervals of 1-3 months, and $\mathrm{HBeAg} / \mathrm{anti}-\mathrm{HBe}$ at 6 months and one year during treatment and 6 months after treatment are recommended. (B1) $\mathrm{HBsAg}$ quantification is recommended pre-treatment, after 12 and 24 weeks of treatment, and at the end of treatment. (B1)

3. Even after virological response, serum HBV DNA measurement is recommended at intervals of 3-6 months. (B1)

4. Monitoring the side effects of each drug during antiviral therapy is necessary. (A1)

\section{CESSATION OF TREATMENT AND MONITOR- ING AFTER ANTIVIRAL TREATMENT}

\section{Clinical biomarkers for treatment endpoint}

In patients with $\mathrm{CHB}$, it is realistically difficult or impossible to determine the appropriate timing of treatment cessation after achieving the ultimate goal of therapy, which is improvement in survival. Therefore, alternative biomarkers that reflect achievement of treatment goals that can easily be measured are needed when evaluating treatment cessation. ALT normalization, undetectable HBV DNA, HBeAg loss or seroconversion and HBsAg loss or seroconversion have been used as treatment endpoints. Cessation of therapy is not recommended in patients with liver cirrhosis because there is a risk of serious liver failure due to relapse and flare after cessation of therapy. ${ }^{67,97}$

The standard treatment duration of peginterferon alfa is 48

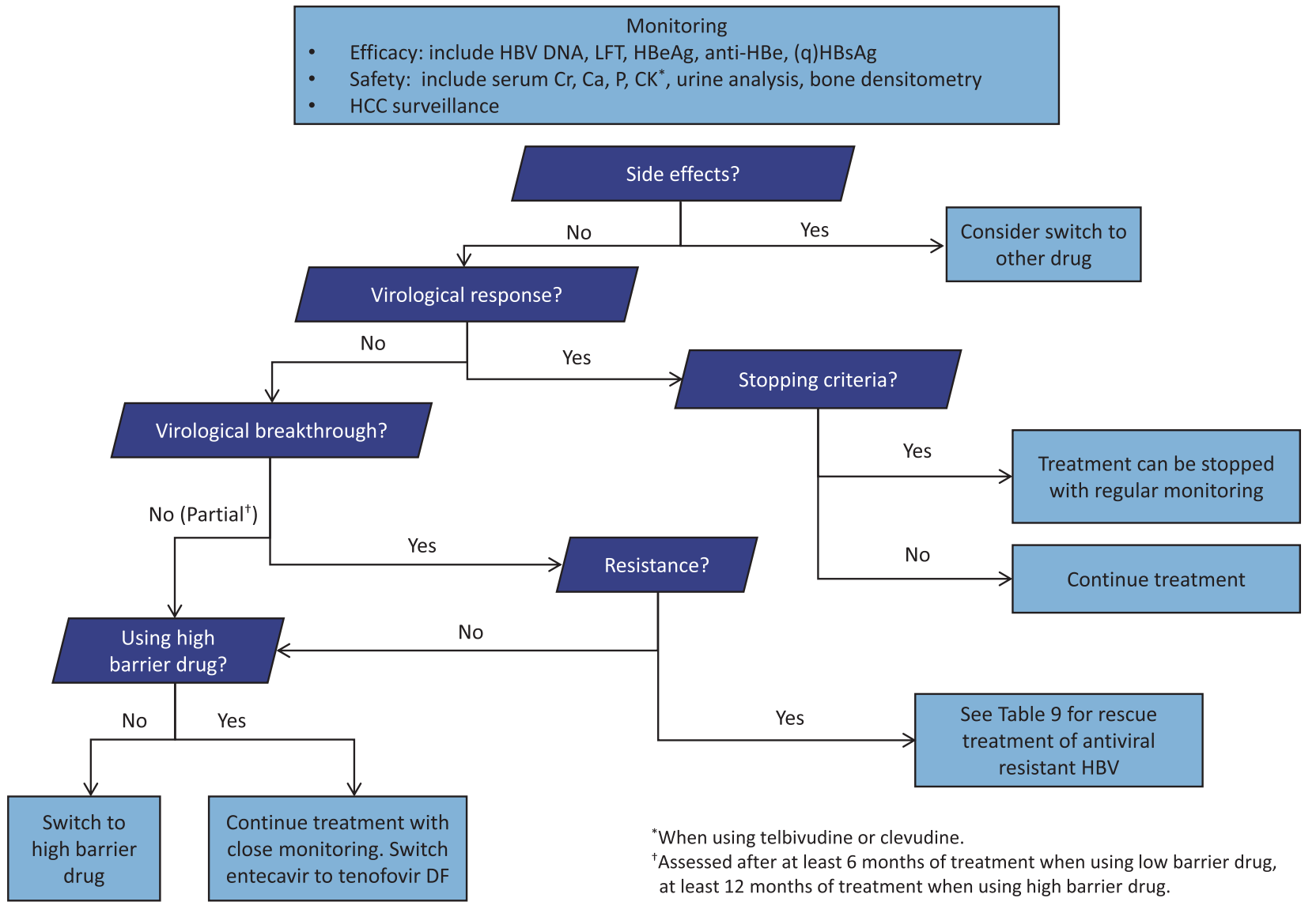

Figure 3. On-treatment management of patients receiving nucleos(t)ide analogues. Appropriate monitoring is required for the proper management at given situations. HBV, hepatitis B virus; LFT, liver function test; HBeAg, hepatitis B e antigen; anti-HBe, antibody to hepatitis B e antigen; HBs Ag, hepatitis B surface antigen; CK, creatine phosphokinase; HCC, hepatocellular carcinoma; tenofovir DF, tenofovir disoproxil fumarate. 
weeks. ${ }^{217,238}$ However, there have been some reports that extended dosing could be more effective in $\mathrm{HBeAg}$-negative $\mathrm{CHB} .{ }^{280,281}$

\section{ALT normalization}

Normalization of ALT in CHB treatment reflects a decrease in hepatic inflammatory response, mostly associated with undetectable HBV DNA, and reduces clinical deterioration. ${ }^{174}$ Normalization of ALT during treatment reflects improvement in cirrhosis and therefore could be considered reflective of treatment goals. ${ }^{149}$

However, $14-40 \%$ of patients with persistently normal ALT could have more than significant fibrosis $(\geq F 2)$ and there are a variety of factors affecting ALT, such as non-alcoholic or alcoholic fatty liver. ${ }^{162,172,282}$ As such, ALT normalization alone is insufficient when determining the endpoint of treatment.

\section{Undetectable HBV DNA}

HBV DNA level is the strongest predictor of disease progression and long-term outcomes in the natural course of $\mathrm{CHB} .{ }^{45,47} \mathrm{HBV}$ DNA levels are associated with histological activity in patients with $\mathrm{CHB}$, and rate of progression to decompensation is low and that of survival is high in patients with low HBV DNA. ${ }^{283,284}$

Antiviral therapy can reduce HBV DNA, and histological improvement can be achieved in proportion to HBV DNA reduction. ${ }^{285}$ When HBV DNA is not detectable for long-term and virological response is well maintained, ${ }^{286,287}$ the HBsAg loss rate increases even after cessation of therapy in $\mathrm{HBeAg}$-negative $\mathrm{CHB}$ patients. ${ }^{286,288}$ Therefore, cessation of therapy could be considered in $\mathrm{HBeAg}$-negative CHB patients with long-term undetectable HBV DNA. ${ }^{289}$ However, in practice, most patients relapsed after cessation of therapy. ${ }^{290-292}$ Hence, undetectable HBV DNA cannot be the sole factor determining treatment cessation.

\section{$\mathrm{HBeAg}$ loss and/or seroconversion}

$\mathrm{HBeAg}$ seroconversion in $\mathrm{HBeAg-positive} \mathrm{CHB}$ is accompanied by HBV DNA reduction, ALT normalization, and improvement in histological findings after antiviral therapy. ${ }^{285,293}$ After HBeAg seroconversion, HBsAg loss also increases to $1.15 \%$ per year. ${ }^{31}$ The incidence of liver cirrhosis and HCC is reduced and survival is improved in both patients with spontaneous or treatment-induced $\mathrm{HBeAg}$ seroconversion compared to patients who are persistently HBeAg positive. 7,15,283,294,295 Therefore, HBeAg loss/seroconversion in $\mathrm{HBeAg-positive} \mathrm{CHB}$ could be considered biomarkers reflecting achievement of treatment goal.

However, HBeAg-negative hepatitis has been reported in $24 \%$ of patients even after HBeAg seroconversion. ${ }^{15}$ Furthermore, the incidence of $\mathrm{HBeAg}$ reversion and an increase in HBV DNA was noted after treatment cessation in patients who achieved HBeAg loss/seroconversion on antiviral therapy. ${ }^{286,287,296,297}$ Therefore, the evidence for recommending treatment cessation depending on $\mathrm{HBeAg}$ loss/seroconversion alone is lacking. Nevertheless, the risk of recurrence is reduced if treatment is discontinued after being maintained for a sufficient period of time (e.g., more than 12 months) after HBeAg loss/seroconversion. ${ }^{298,299}$

\section{HBsAg loss}

$\mathrm{HBsAg}$ levels quantified by qHBsAg assay reflect the natural course of disease in patients with $\mathrm{CHB}^{112}$ and are also proportional to the levels of cccDNA in the liver. ${ }^{300} \mathrm{HBsAg}$ levels may decrease after HBeAg loss during antiviral therapy. ${ }^{259}$ The incidence of $\mathrm{HCC}$ is significantly reduced when HBsAg loss occurs before age 45-50. ${ }^{23,301}$ Some patients with HBsAg loss/conversion during antiviral therapy showed HBsAg reversion or low but detectable HBV DNA, but most patients maintain HBsAg loss and undetectable HBV DNA levels, and their incidence of HCC is significantly lower compared to patients without HBsAg loss. ${ }^{154,302}$ Therefore, HBsAg loss is the ideal endpoint of antiviral therapy in $\mathrm{CHB}$, reflecting the treatment goal, at which point NAs can be discontinued. Recently, it has been reported that HBsAg reversion can be better avoided if antiviral therapy is discontinued after maintaining treatment for 6-12 months or longer despite HBsAg loss. ${ }^{302}$

However, HBsAg loss is very rare and long-term treatment (50 years or longer) is required based on the decreasing dynamics of HBsAg levels during treatment with NAs. ${ }^{303,304}$ Despite HBsAg loss, there is always a risk of developing HCC and surveillance is still necessary. ${ }^{301,305,306}$ It remains unclear whether HBsAg loss will further improve the long-term clinical prognosis beyond that expected by undetectable HBV DNA.

\section{Monitoring after antiviral treatment}

The response to antiviral treatment persists in some patients, while others relapse. Therefore, regular monitoring of liver function tests, HBeAg, anti-HBe, and HBV DNA is needed to evaluate the durability of the treatment response, relapse, and deterioration in liver function. qHBsAg levels may be helpful in monitoring $\mathrm{HBsAg}$ reduction or loss in patients without HBsAg loss after cessation of therapy. Even in patients in whom HBsAg loss has been achieved, there is the potential risk for reversion of $\mathrm{HBsAg}$ or development of HCC. ${ }^{154,301,302}$ Therefore, serum HBsAg and/or antiHBs should be monitored and HCC surveillance should be performed continuously. 


\section{[Recommendations]}

1. Cessation of NAs is recommended after serum HBsAg loss in CHB patients. (A1)

2. In $\mathrm{HBeAg}$-positive CHB patients, cessation of NA therapy could be considered at least 12 months after HBV DNA is undetectable and serum $\mathrm{HBeAg}$ loss or seroconversion has been achieved. (B2)

3. Long-term treatment should be considered in patients with liver cirrhosis. Indefinite NA therapy is recommended in patients with decompensated liver cirrhosis. (B1)

4. Peginterferon alfa is administered for 48 weeks. (A1)

5. Liver function testing and serum HBV DNA measurement at 1-6-month intervals and $\mathrm{HBeAg} / \mathrm{anti}-\mathrm{HBe}$ testing at 3-6-month intervals are recommended during the first year after cessation of antiviral treatment. Liver function testing and serum HBV DNA measurement at 3-6-month intervals and $\mathrm{HBeAg} /$ anti-HBe testing at 6-12-month intervals are recommended if treatment response is maintained beyond one year after antiviral therapy. (B1)

6. If virological response is maintained after cessation of antiviral treatment, follow-up HBsAg/anti-HBs testing should be performed to confirm HBsAg loss, maintenance, or reversion. (B1)

\section{ANTIVIRAL RESISTANCE}

The development of antiviral resistance decreased significantly after the use of drugs with high genetic barriers such as entecavir and tenofovir (including tenofovir DF and tenofovir AF) as firstline treatments. Nonetheless, antiviral resistance is an important factor that determines success or failure of CHB treatment. The emergence of antiviral resistance results in resumption of active viral replication that had been suppressed by antiviral therapy and can impair biochemical or histological improvement. Therefore, prevention, early diagnosis, and management of antiviral resistance may significantly affect the long-term prognosis of CHB patients undergoing antiviral therapy. ${ }^{307-309}$

\section{Mechanism of antiviral resistance and definitions}

Mutations in HBV can occur in all four open reading frames (ORF) of pres/S, polymerase, precore/core, and X. Among the mutations in the polymerase ORF, which is the target of several NAs, those that can replicate under the influence of the antiviral agent are selected, and the ratio is increased. Although the first-occurring antiviral-resistant mutant has decreased replication capacity, a compensatory mutation develops over time that restores replication capacity to the wild-type level. ${ }^{310}$ Selection for a particular mutation affects both resistance to the drug being administered and the replication capacity.

A genetic barrier is defined as the number of genetic mutations needed to develop antiviral resistance, with a higher genetic barrier indicating a lower risk of resistance. ${ }^{311}$ The antiviral potency of drugs also influences the development of resistance. Therefore, as a first-line treatment, it is important to use drugs with a high barrier to HBV resistance such as entecavir, tenofovir, and besifovir, which have a high inhibitory effect on virus proliferation, or, alternately, peginterferon alfa. If other drugs have been used, careful monitoring for resistance development is required.

\section{Mutations conferring resistance to antiviral agents}

Antiviral agents used in treatment of HBV infection are classified into two groups: nucleoside analogues (L-nucleoside analogues [lamivudine, telbivudine, and clevudine] and cyclopentenes [entecavir]) and nucleotide analogues (acyclic phosphonates [adefovir, tenofovir, besifovir]). ${ }^{312}$ Cross-resistance between nucleosides and nucleotide analogues is rarely observed. Tables 7 and 8 summarize the types and frequencies of known drug resistance mutations.

\section{Nucleoside analogues}

L-nucleoside analogues (lamivudine, telbivudine, and clevudine): All L-nucleosides have a similar molecular structure and target site of action, resulting in similar patterns of antiviral resistance mutations. Mutations at rtM204 are the primary resistance mutations to L-nucleosides. ${ }^{309,313-315}$ The rtM204V and rtM204I mutations involve substitution of methionine with valine and isoleucine, respectively, at codon 204 of the reverse transcriptase gene. ${ }^{316}$ Originally, these were termed YMDD mutations. $^{316}$ The specific primary mutations conferring resistance are rtM204V/I substitutions for lamivudine and only the rtM204I substitution for telbivudine and clevudine. ${ }^{178,317-319}$ An rtM204V mutant may commonly accompany rtL180M but not rtM204I. ${ }^{320}$ These mutants are sensitive to nucleotide analogues, but they exhibit cross-resistance to entecavir and an eight-fold decrease in sensitivity. The rtA181T mutation has been detected in 5\% of lamivudine-resistant patients, in whom susceptibility to telbivudine and clevudine is also reduced. This mutation confers concomitant resistance to adefovir, but these mutants remain susceptible to entecavir. ${ }^{321}$

Cyclopentene (entecavir): Resistance to entecavir occurs through a two-hit mechanism. rtL180M and rtM204V first develop as background mutations, and then additional mutations such as rtT184L/F/A/M/S/I/C/G, rtS202G/I/C, or rtM250V/I/L develop as 
Table 7. Hepatitis B virus (HBV) reverse transcriptase (RT) gene mutations associated with antiviral resistance

\begin{tabular}{|c|c|c|c|c|c|}
\hline HBV variant & LAM & LdT & ETV & ADV & TDF/TAF \\
\hline Wild-type & $S$ & $S$ & $S$ & S & S \\
\hline M204V & R & S & । & । & S \\
\hline M204I & $\mathrm{R}$ & $\mathrm{R}$ & I & । & S \\
\hline L180M+M204V & $\mathrm{R}$ & R & । & । & S \\
\hline A181T/V & । & । & S & R & । \\
\hline $\mathrm{N} 236 \mathrm{~T}$ & S & S & S & $\mathrm{R}$ & I \\
\hline L180M+M204V/I+I169T or V173L or M250V & R & $\mathrm{R}$ & R & S & S \\
\hline L180M+M204V/I+184G or S202I/G & $\mathrm{R}$ & R & R & S & $S$ \\
\hline
\end{tabular}

The amino acid substitution profiles are shown in the left column and the level of susceptibility is given for each drug: S (sensitive), I (intermediate/reduced susceptibility), R (resistant). Reports on mutations associated with TDF resistance - L180M+M204V+A194T in human immunodeficiency virus (HIV)/HBV coinfection patients, ${ }^{330} \mathrm{~S} 106 \mathrm{C}+\mathrm{H} 126 \mathrm{Y}+\mathrm{D} 134 \mathrm{E}+\mathrm{L} 269 \mathrm{I}$ in HBV patients. ${ }^{333}$

LAM, lamivudine; LdT, telbivudine; ETV, entecavir; ADV, adefovir; TDF, tenofovir disoproxil fumarate; TAF, tenofovir alafenamide fumarate.

Table 8. Cumulative incidence of antiviral resistance development from representative studies

\begin{tabular}{|c|c|c|c|c|c|c|c|c|}
\hline \multirow{2}{*}{ Antiviral agent } & \multicolumn{8}{|c|}{ Resistance rate (\%) } \\
\hline & Year 1 & Year 2 & Year 3 & Year 4 & Year 5 & Year 6 & Year 7 & Year 8 \\
\hline Lamivudine $^{\ddagger}$ & 24 & 42 & 53 & 70 & $\geq 65$ & & & \\
\hline Telbivudine $e^{\S}$ & $2.7-4.4$ & $10.8-25.1$ & & & & & & \\
\hline Clevudine & 2.3 & 24.4 & 30.0 & & & & & \\
\hline \multicolumn{9}{|l|}{ Adefovir } \\
\hline In treatment-naïve $e^{*, * *}$ & 0 & 3 & 11 & 18 & 29 & & & \\
\hline In lamivudine resistant ${ }^{\dagger \dagger}$ & $4.4-18$ & $18.4-25$ & 34.3 & 52.3 & 65.6 & & & \\
\hline
\end{tabular}

Adefovir+lamivudine

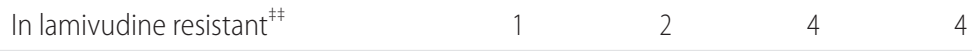

\begin{tabular}{|c|c|c|c|c|c|c|c|c|}
\hline \multicolumn{9}{|l|}{ Entecavir } \\
\hline In treatment-naïve ${ }^{\S \S}$ & 0.2 & 0.5 & 1.2 & 1.2 & 1.2 & 1.2 & 1.2 & \\
\hline In lamivudine refractory ${ }^{||||}$ & 6 & 15 & 36 & 47 & 51 & & & \\
\hline Tenofovir DF ${ }^{\dagger * * * *}$ & 0 & 0 & 0 & 0 & 0 & 0 & 0 & 0 \\
\hline Tenofovir $\mathrm{AF}^{\dagger+t}$ & 0 & 0 & & & & & & \\
\hline Besifovir ${ }^{\ddagger \neq}$ & 0 & 0 & & & & & & \\
\hline
\end{tabular}

Tenofovir DF, tenofovir disoproxil fumarate; Tenofovir AF, tenofovir alafenamide fumarate.

*Hepatitis B e antigen (HBeAg)-negative patients.

${ }^{\dagger}$ Emtricitabine was combined in patients with detectable hepatitis B virus deoxyribonucleic acid (HBV DNA) after 72 weeks of treatment.

${ }^{\ddagger}$ Modified and updated from Lai et al. ${ }^{309}$ and Lok et al. ${ }^{313}$

${ }^{\S}$ Modified and updated from Lai et al. ${ }^{178}$ and Liaw et al. ${ }^{317}$

"Modified and updated from Yoon et al. ${ }^{319}$

** Modified and updated from Hadziyannis et al. ${ }^{325}$

${ }^{\dagger \dagger}$ Modified and updated from Lee et al. ${ }^{337}$, Yeon et al. ${ }^{338}$, and Lee et al. ${ }^{272}$

${ }^{\ddagger \ddagger}$ Modified and updated from Lampertico et al. ${ }^{328}$

${ }^{\S}$ Modified and updated from Tenney et al. ${ }^{323}$ and Lam et al. ${ }^{231}$

III Modified and updated from Tenney et al. ${ }^{323}$

*** Modified and updated from Liu et al. ${ }^{233}$

${ }^{t+\dagger}$ Modified and updated from Agarwal et al. ${ }^{224}$

${ }^{\ddagger \neq \ddagger}$ Modified and updated from Yuen et al. ${ }^{335}$ 
primary resistance mutations to entecavir, resulting in a marked decrease in drug susceptibility. ${ }^{311,322}$ rtl169T is a compensatory mutation that increases the fold resistance of rtT184, rtS202, and rtM250 mutants. Since multiple genetic mutations are needed to develop high-level resistance to entecavir (high genetic barrier), the resistance rate in treatment-naive CHB patients is very low. However, a resistance rate as high as $51 \%$ has been reported after five years of treatment in lamivudine-refractory subjects. ${ }^{323}$ In addition, exposure to lamivudine increases the risk of resistance to entecavir even if no previous resistance has occurred. ${ }^{324}$ Thus, it is important to use drugs with a high barrier to HBV resistance as first-line treatment.

\section{Nucleotide analogues}

Adefovir: rtN236T and rtA181V/T are the primary resistance mutations to adefovir. ${ }^{325,326}$ The levels of resistance of rtN236T and rtA181T to adefovir are 7- to 10-fold and 2.5- to 5-fold, respectively, compared to the wild-type virus. ${ }^{312,321}$ rtA181T can be detected in subjects receiving lamivudine monotherapy or combination therapy comprising adefovir plus lamivudine. ${ }^{327,328}$

Tenofovir: rtA194T can decrease susceptibility to tenofovir by 6.9- to 10 -fold in the presence of rtL180M+rtM204V mutations. ${ }^{329-331}$ It has been reported that rtS78T/sC69* inhibits tenofovir sensitivity 1.6-fold, ${ }^{332}$ but its clinical significance needs to be confirmed. Recently, a novel mutation was found in sera of patients with viral breakthrough in the treatment of tenofovir DF in Korea. ${ }^{333}$ In a laboratory phenotypic resistance study, rtS106C+ rtH126Y+rtD134E+rtL269l conferred a 15.3-fold increase in resistance to tenofovir.

Besifovir: In a multicenter clinical study, viral breakthrough was observed in patients with poor compliance, but no mutations associated with resistance of besifovir were observed. ${ }^{230,334,335}$

\section{MANAGEMENT OF ANTIVIRAL RESISTANCE}

Prior antiviral resistance predisposes individuals to subsequent viral mutations and limits the choice of rescue therapies due to cross-resistance. ${ }^{312,336}$ In addition, even if antiviral agents without cross-resistance are selected, resistance to rescue therapy is more frequent than in treatment-naïve subjects. ${ }^{336-338}$ Careful selection of a first-line antiviral agent is essential to minimize resistance and cross-resistance to other agents.

Antiviral resistance testing is required when a virological or biochemical breakthrough is detected in subjects with good compli- ance. In cases of resistance, an appropriate rescue therapy should be initiated with the most effective antiviral agent without crossresistance to minimize the risk of inducing multiple drug-resistant strains. ${ }^{339}$ Table 9 shows recommendations for treatment adaptation.

\section{Management of nucleoside analogue resistance}

Patients with confirmed resistance to nucleoside analogues such as lamivudine, telbivudine, clevudine, and entecavir can be changed to treatment with tenofovir alone. A prospective study showed that tenofovir DF monotherapy was highly efficacious in patients with lamivudine-resistant HBV and comparable to the combination of tenofovir DF and emtricitabine, without emergence of additional resistance mutations to tenofovir DF throughout 96 weeks of treatment. ${ }^{340}$ Although data on rescue therapy with telbivudine and clevudine are lacking, their resistance mutations are very similar, and we recommend that treatment be based on lamivudine resistance. A recent multicenter randomized trial with patients who had lamivudine/entecavir-resistant HBV, found that the proportion of patients with HBV DNA $<15 \mathrm{IU} / \mathrm{mL}$ was not significantly different between the tenofovir DF and tenofovir DF/ entecavir groups (71\% vs. $73 \%) .{ }^{341} \mathrm{~A}$ retrospective study that compared tenofovir DF with tenofovir DF/entecavir among patients who had lamivudine/entecavir-resistant HBV found that the HBV undetectable rate up to 24 months (HBV DNA $<20 \mathrm{IU} / \mathrm{mL}$ ) did not differ significantly between groups (85.4\% vs. $89.2 \%$ ). ${ }^{342}$ However, tenofovir DF/entecavir combination therapy was superior to tenofovir DF monotherapy in patients with a high baseline viral load (HBV DNA >4 log $\mathrm{IU} / \mathrm{mL}$ ).

\section{Management of nucleotide analogue resistance}

Switching to tenofovir monotherapy or tenofovir/entecavir combination therapy is recommended in cases of adefovir resistance. In vitro studies showed that susceptibility of adefovir-resistant HBV with a single rtN236T or rtA181V/T mutation to tenofovir is similar to that of wild-type HBV, but susceptibility is lower when both mutations are present. ${ }^{321}$ Clinically, most studies have found that tenofovir is effective in suppressing adefovir-resistant HBV. Tenofovir DF alone or tenofovir DF and emtricitabine are similarly effective in patients with CHB treated with adefovir (82\% vs. $84 \%){ }^{343}$ However, this study reported that only $27.6 \%$ of patients had HBV with adefovir resistance mutations. A recent multicenter randomized trial with patients who had adefovir-resistant HBV 
found that the proportion of patients with HBV DNA $<15 \mathrm{IU} / \mathrm{mL}$ was not significantly different between those treated with tenofovir DF and tenofovir DF/entecavir (62.0\% vs. 63.5\%). ${ }^{344}$ When these patients were followed for three years, there was no difference in incidence of undetectable HBV DNA level between the two groups. However, the decrease in serum HBV DNA level tended to be smaller in a subgroup of patients with HBV that had both adefovir resistance mutations, (rtA181T/V and rtN236T), though longer follow-up is needed to confirm this finding. ${ }^{345} \mathrm{~A}$ Korean retrospective study reported that tenofovir DF had inferior efficacy in adefovir-experienced CHB patients. ${ }^{346}$ Thus, CHB patients with a history of adefovir exposure should be monitored closely for response to tenofovir monotherapy and virological breakthrough.

We recommend adding entecavir to tenofovir in cases of tenofovir resistance. However, if the resistance is accompanied by that to entecavir, treatment with a nucleos(t)ide antiviral agent is difficult, indicating the need for a new therapeutic agent.

\section{Management of multidrug resistance}

Although there is no clear international definition, management of multidrug resistance (MDR) is defined as resistance to two or more classes of antiviral drugs. ${ }^{336}$ This is due to the low efficacy of adefovir and entecavir used as previous rescue therapy for L-nucleoside analogue resistant-HBV and their sequential therapy alone. Most studies on MDR management have few patients with heterogeneous resistance mutations and a diverse combination of therapies. Although there is no established treatment, tenofovir/ entecavir combination therapy, tenofovir monotherapy, and adefovir/entecavir combination therapy are the preferred treatment options. ${ }^{347}$ The serum HBV DNA non-detection rate $(<12 \mathrm{lU} / \mathrm{mL})$ was reported to be $62.5 \%$ in a prospective multicenter study of 64 patients with MDR CHB after 48 weeks of treatment with tenofovir DF/entecavir combination therapy. ${ }^{348}$ Tenofovir DF monotherapy had non-inferior antiviral efficacy compared with tenofovir DF-based combination therapy in MDR CHB patients in a multicenter cohort studies. ${ }^{47,349}$ In addition, there was no significant difference in virological response at 48 weeks between tenofovir DF monotherapy and tenofovir DF/entecavir combination therapy (66.3\% vs. 68.0\%) in CHB patients with resistance mutations to ETV and/or adefovir. ${ }^{345}$ In this regard, patients with MDR CHB may be treated with tenofovir monotherapy.

There has been a recent report of tenofovir resistance in Korea. ${ }^{350}$ Also, a prospective study found that approximately onequarter of patients, particularly those with adefovir-resistant $\mathrm{CHB}$, did not have a satisfactory virological response until three years of rescue therapy. ${ }^{345}$ It is therefore necessary to identify factors predictive of treatment response and to verify long-term therapeutic effects.

\section{[Recommendations]}

1. If a virological breakthrough occurs during NA treatment, patient medication compliance should be assessed, and antiviral resistance testing should be performed. (A1)

2. Rescue therapy for antiviral resistance should be initiated as soon as possible once a virological breakthrough is detected and genotypic resistance is confirmed. (A1)

3. For CHB resistant to L-nucleoside analogues such as lamivudine, telbivudine, and clevudine, switch to tenofovir monotherapy. (A1)

4. For entecavir-resistant $\mathrm{CHB}$, switch to tenofovir monotherapy, or add tenofovir. (A1)

Table 9. Rescue treatment of antiviral-resistant hepatitis B virus

\begin{tabular}{|c|c|c|}
\hline Resistance & Preferred & Alternative \\
\hline Lamivudine/Telbivudine/Clevudine resistance & 1. Change to tenofovir ${ }^{\dagger}$ & $\begin{array}{l}\text { 1. Add tenofovir }{ }^{\dagger} \\
\text { 2. Add ADV }\end{array}$ \\
\hline ETV resistance & $\begin{array}{l}\text { 1. Change to tenofovir }{ }^{\dagger} \\
\text { 2. Add tenofovir }\end{array}$ & 1. Add ADV \\
\hline ADV resistance & $\begin{array}{l}\text { 1. Change to tenofovir }{ }^{\dagger} \\
\text { 2. Change to ETV+tenofovir }\end{array}$ & 1. Add ETV \\
\hline Tenofovir resistance & 1. Add ETV & \\
\hline Multi-drug resistance & $\begin{array}{l}\text { 1. Change to ETV+tenofovir } \\
\text { 2. Change to tenofovir }\end{array}$ & \\
\hline
\end{tabular}

$A D V$, adefovir; ETV, entecavir.

*Preferred for heavily pretreated patients (e.g. including ETV+ADV).

${ }^{\dagger}$ Tenofovir disoproxil fumarate (TDF) or tenofovir alafenamide fumarate (TAF). 
5. For adefovir-resistant $\mathrm{CHB}$, switch to tenofovir monotherapy or tenofovir and entecavir combination therapy. (A1)

6. For tenofovir-resistant CHB, add entecavir. (B1)

7. For multidrug-resistant $\mathrm{CHB}$, switch to tenofovir and entecavir combination therapy or tenofovir monotherapy. (A1)

\section{MANAGEMENT ACCORDING TO TREATMENT RESPONSE}

Persistent viral replication during antiviral treatment for CHB is a risk factor for the progression of hepatic fibrosis and the development of antiviral-resistant mutations. Thus, treatment response should be evaluated by measuring serum HBV DNA levels with sensitive real-time PCR methods at 3-6-month intervals. Even for patients who achieved virological response, treatment response needs to be monitored at 3-6 month intervals until the patient is able to stop medication after achieving the treatment goal. ${ }^{109}$ When patients under peginterferon alfa treatment show insufficient virological response, early cessation of treatment can be considered.

\section{Management of partial virological response to NAs}

Although there have been few studies on partial virological response, it is recommended to switch from one NA to other NA options with no cross-resistance and a high genetic barrier for treatment-adherent patients with partial virological response (Fig. 3). Upon switching to entecavir $1 \mathrm{mg}$ per day in patients with a partial virological response to lamivudine, $67.6 \%$ of patients demonstrated an undetectable HBV DNA $(<60 \mathrm{IU} / \mathrm{mL})$ rate at week 96. ${ }^{351}$ However, as lamivudine-experienced patients treated with entecavir showed a relatively higher risk of developing entecavirresistant mutation in lamivudine-experienced patients, ${ }^{324}$ caution is needed when switching to entecavir. In contrast, tenofovir was reported to provide good antiviral efficacy regardless of prior exposure or resistance to lamivudine. ${ }^{336}$ In the case of partial virological response to either entecavir or tenofovir, which have a high genetic barrier, medication can be maintained if serum HBV DNA decreases continuously, since the risk of developing a resistant mutation is low and delayed virological response can be expected without changing the antiviral regimen. However, switching to another drug might be considered if the decrease in HBV DNA is not remarkable within 12 months (Fig. 3). ${ }^{97}$ Recently, a prospective randomized trial in Korea reported that upon switch- ing to tenofovir DF, $55 \%$ of patients with partial virological response to 12 months of entecavir treatment showed undetectable serum HBV DNA ( $<20 \mathrm{IU} / \mathrm{mL})$ at month 12 , compared to $20 \%$ in an entecavir maintaining group. ${ }^{352} \mathrm{~A}$ meta-analysis also reported that switching to tenofovir DF is effective for those patients. ${ }^{353}$ Prior research on the optimal treatment strategy for partial virological response to tenofovir has been highly limited. In a Korean study, $90.2 \%$ of patients who showed partial virological response to tenofovir DF treatment at month 12 achieved virological response at year three when they continued tenofovir DF treatment. ${ }^{354}$

\section{Management after achieving virological response with NA treatment}

Tenofovir DF monotherapy showed comparable antiviral efficacy to tenofovir DF/entecavir combination therapy for patients with entecavir- or adefovir-resistant mutations in prospective randomized trials. ${ }^{341,344,345,349,355}$ Based on these studies, a switch to tenofovir monotherapy can be considered in patients achieving virological response to rescue combination therapy with tenofovir and entecavir. Since long-term treatment is currently inevitable for $\mathrm{CHB}$, the potential benefit and risk, or cost of combination therapy should be weighed. A Korean retrospective study reported that virological response was sustained in all 76 patients during a median 2-year follow-up when the rescue treatment was switched from combination treatment with tenofovir DF plus entecavir to tenofovir DF monotherapy. ${ }^{356}$

The ideal endpoint of antiviral treatment for CHB patients is a functional cure with HBsAg loss. Although HBsAg can be lost with NAs alone, the annual rate was as low as $0.8 \%{ }^{357}$ Therefore, there have been a number of studies on additional interferon treatment and/or therapeutic vaccination on NAs to maximize treatment effect.

Several randomized controlled trials reported 4-9.8\% HBsAg seroclearance rates when NA was changed to 48-week peginterferon alfa treatment with/without maintaining NA. ${ }^{358-362}$ In addition, 96-week peginterferon alfa treatment increased the HBsAg seroclearance rate to $15.3 \%$. However, another study showed no difference in $\mathrm{HBSAg}$ seroclearance rate between the adding peginterferon alfa group $(n=92)$ and continuing NA group $(n=93)$ (7.8\% vs. $3.2 \%$; $P=0.15$ ) in $\mathrm{HBeAg}$-negative patients achieving virological response with NAs. ${ }^{363}$ A recent Korean randomized controlled study reported a $16.2 \% \mathrm{HBsAg}$ seroclearance rate with an additional 48-week peginterferon alfa-2a treatment period (180 $\mu \mathrm{g}$ every week) and sequential HBV vaccination in patients 
who achieved virological response with entecavir and qHBsAg was $<3,000 \mathrm{IU} / \mathrm{mL} .^{364}$ However, although additional treatment with interferon and/or therapeutic vaccination can be an option to increase the chance of HBsAg loss, the treatment benefit of combination or sequential therapy over monotherapy with NAs is unclear in Korea, where genotype C HBV is prevalent. Further studies comparing treatment benefit and cost or adverse event of those additional treatments are warranted prior to applying those additional treatment strategies in clinical practice.

\section{Management of suboptimal response to peginterferon alfa}

Serum qHBsAg titer is a good predictor of response to peginterferon alfa and is utilized as a stopping rule. ${ }^{67,97}$ In HBeAg-positive CHB patients, a decline of qHBsAg levels below 1,500 IU/mL at 12 weeks in a reasonable predictor of $\mathrm{HBeAg}$ seroconversion. However, qHBsAg levels $>20,000 \mathrm{IU} / \mathrm{mL}$ at 12 or 24 weeks are associated with a very low probability of subsequent HBeAg seroconversion and can be considered as peginterferon alfa stopping rules. ${ }^{16,256}$

In HBeAg-negative CHB patients, a decline of HBV DNA by $\geq 2$ $\log _{10} \mathrm{IU} / \mathrm{mL}$ at 12 weeks and $\mathrm{a} \geq 10 \%$ decline in serum $\mathrm{qHBsAg}$ from baseline to week 12 had a higher probability of achieving a sustained response. However, the combination of a lack of decrease in qHBsAg levels and $<2 \log _{10} \mathrm{IU} / \mathrm{mL}$ decline in HBV DNA at 12 weeks predicts no treatment response and are considered peginterferon alfa stopping rules. . $^{25,255,365}$

\section{[Recommendations]}

1. Compliance with medication should be carefully monitored in patients with a partial virological response to NA therapy. (A1)

2. In CHB patients with a partial virological response to NAs with low genetic barriers, switching to NAs with high genetic barriers and no cross-resistance is recommended. (A1)

3. In CHB patients with a partial virological response to NAs with high genetic barriers, treatment can be continued while monitoring virological responses at 3-6-month intervals. (B1) However, in the case of partial virological response to entecavir, switching to tenofovir can be considered. (A2)

4. During peginterferon alfa treatment, $\mathrm{qHBsAg}$ levels $>20,000$ $\mathrm{IU} / \mathrm{mL}$ after 24 weeks of therapy in $\mathrm{HBeAg}$-positive $\mathrm{CHB}$ patients as well as a combination of stable qHBsAg levels and a reduction in serum HBV DNA levels to less than $2 \log _{10} \mathrm{IU} / \mathrm{mL}$ after 12 weeks of therapy in $\mathrm{HBeAg-negative} \mathrm{CHB} \mathrm{patients}$ are associated with a very low probability of subsequent treatment response, and cessation of therapy should be considered. (B2)

\section{MANAGEMENT IN SPECIAL CONDITIONS}

\section{Patients with HCC}

The aims of antiviral treatment in patients with HBV-related HCC are: i) the suppression of HBV replication to prevent the progression of hepatic dysfunction, thereby enabling active treatment of HCC and ii) the reduction of HCC recurrence after potentially curative treatment. $^{97}$

Antiviral treatment after curative treatment of $\mathrm{HCC}$

There have been several studies that reported antiviral treatment was associated with lower risk of tumor recurrence after curative treatment (i.e., surgical resection, radiofrequency ablation [RFA], and percutaneous ethanol injection) for HBV-related HCC. A Taiwanese large-scale retrospective study reported that patients who underwent NA treatment with entecavir, lamivudine, telbivudine, etc. showed a significantly lower risk of tumor recurrence or overall death after surgical resection for HCC, although the prevalence of cirrhosis was significantly higher. ${ }^{366}$ Even in patients with low-level viremia (HBV DNA $<2,000 \mathrm{IU} / \mathrm{mL}$ ), antiviral treatment was associated with longer recurrence-free survival $(P=0.016)$ and overall survival $(P=0.004) .{ }^{367}$ A meta-analysis showed that the antiviral treatment group had significantly lower risk of tumor recurrence (odds ratio $[\mathrm{OR}], 0.59 ; 95 \%$ confidence interval $[\mathrm{Cl}$, $0.35-0.97 ; P=0.04)$, liver-related mortality $(\mathrm{OR}, 0.13 ; 95 \% \mathrm{Cl}$, $0.02-0.69 ; P=0.02)$, and overall mortality $(\mathrm{OR}, 0.27 ; 95 \% \mathrm{Cl}$, $0.14-0.50 ; P<0.001)$, than the no antiviral treatment group. ${ }^{368}$ In a retrospective Korean study, antiviral treatment with high-potency NAs (i.e., entecavir and tenofovir) showed significantly longer recurrence-free survival than both antiviral treatment with lowpotency NAs (i.e., lamivudine, clevudine, and telbivudine) and no antiviral treatment. ${ }^{369}$ In contrast, a randomized controlled trial reported that adjuvant interferon alfa-2b treatment was not associated with lower risk of post-resection tumor recurrence $(P=0.828){ }^{370}$

\section{Antiviral treatment during $\mathrm{HCC}$ treatment}

An increase in serum HBV DNA or HBV reactivation accompanied by abnormalities on biochemical liver function testing has been observed in $14-32 \%$ of CHB patients who undergo surgical resection for $\mathrm{HCC}^{371}$ A prospective study reported that the HBV reactivation rate after surgical resection was $2.5 \%$ in patients that underwent antiviral prophylaxis with telbivudine and $31.8 \%$ in controls. $^{372}$

The post-RFA risk of HBV reactivation was reported to be 5.6- 
9.1\%, whereas no reactivation was reported after percutaneous ethanol injection. ${ }^{37,374}$ After transarterial chemoembolization (TACE), approximately $4-40 \%$ of patients with HBV-related HCC developed HBV reactivation. ${ }^{375-380}$ Post-TACE risks of HBV reactivation, flare-up hepatitis, and liver failure due to HBV reactivation were $2.8 \%, 2.8 \%$, and $0 \%$, respectively, in the prophylactic lamivudine treatment group and $40.5 \%, 29.7 \%$, and $8.1 \%$ in the control group. There was a significant difference between the two groups. ${ }^{378}$ After hepatic artery infusion chemotherapy (HAIC), HBV reactivation was reported in $24-67 \%$ of patients, which was relatively higher than after TACE. This finding can potentially be explained by a larger total amount of cytotoxic chemotherapeutic agent due to shorter treatment interval than TACE. ${ }^{381,382}$

In patients who underwent external beam radiation therapy (EBRT) for HCC, reactivation and ALT elevation were reported in $0 \%$ and $2.3 \%$, respectively, of the lamivudine-prophylaxis group, and $21.8 \%$ and $12.5 \%$, respectively, of the control group. The control group had significantly higher risk of HBV reactivation. ${ }^{383}$ The combination treatment with TACE and EBRT had twice the risk of HBV reactivation compared to TACE treatment alone. ${ }^{384}$ The incidence of HBV reactivation after cytotoxic chemotherapy was reported to be $30-60 \%, 30 \%$ of which resulted in death. ${ }^{380,381}$

In CHB patients, prophylactic antivirals should be maintained for at least 6 months after cessation of cytotoxic chemotherapy and life-long administration is recommended. Interferon is not recommended as a prophylactic antiviral due to bone marrow suppression and transient hepatitis aggravation. There was no reactivation reported during sorafenib treatment in a retrospective study, ${ }^{385}$ while another study reported a high risk of HBV reactivation. ${ }^{386}$ Thus, further observational studies are warranted. Immune checkpoint inhibitors, such as nivolumab and pembrolizum$a b$, may enhance host immunity and consequently have a lower risk of HBV reactivation. However, immune checkpoints can result in severe acute aggravation of hepatitis since it can upregulate antiviral immunity against HBV. Therefore, suppression of HBV replication with antiviral treatment is necessary before use of immune checkpoint inhibitors. ${ }^{387}$

In conclusion, HBV reactivation after various treatments for HBV-related HCC is frequently observed and prophylactic antiviral treatment can reduce the risk of HBV reactivation. Thus, prophylactic antiviral treatment is recommended for patients who undergo surgical treatment, locoregional treatment, radiation treatment, and systemic treatment for HBV-related HCC, regardless of detectable serum HBV DNA.

\section{[Recommendations]}

1. In patients with HBV-related HCC, antiviral therapy should be initiated if serum HBV DNA is detected. (A1)

2. In patients with HBV-related HCC who undergo anticancer treatment, prophylactic antiviral therapy with NAs should be considered regardless of detectable serum HBV DNA. (B1)

\section{Patients with renal dysfunction or metabolic bone disease}

Long-term administration of adefovir or tenofovir DF in the patients with $\mathrm{CHB}$, may result in decreased renal function and bone mineral density. Side effects such as acute on chronic renal failure, hypophosphatemia, and Fanconi syndrome have been reported. ${ }^{27,388-390}$ If patients already have risk factors for renal dysfunction and/or metabolic bone disease, or if worsening kidney function or bone disease is detected during treatment, a change in treatment regimens needs to be considered.

Patients with renal dysfunction or metabolic bone disease prior to starting the treatment

Patients with chronic kidney disease are known to have relatively higher rate of exposure to HBV infection. ${ }^{391}$ In Korea, around $5 \%$ of HBsAg positive rate has been reported among the hemodialysis patients. ${ }^{392-396}$ When starting the oral antiviral agents in the patients with chronic kidney disease, we need to adjust the dose according to the creatinine clearance (Table 10). If creatinine clearance is below $15 \mathrm{~mL} / \mathrm{min}$ without renal replacement therapy, tenofovir AF is not recommended. Same goes for besifovir in case of creatinine clearance below $50 \mathrm{~mL} / \mathrm{min}$. Tenofovir DF is not recommended in the patients with both creatinine clearance below $10 \mathrm{~mL} / \mathrm{min}$ and no renal replacement therapy.

Because nucleotide analogue treatment itself may affect renal function or bone density, it is necessary to select an appropriate drug if there is any risk factor. In a large Phase 3 trial comparing tenofovir AF and tenofovir DF over the 96-week treatment period, among those with any risk factor such as renal insufficiency, decreased bone density, old age, diabetes, or hypertension, patients treated with tenofovir DF had worsening renal function and bone density compared to patients treated with tenofovir AF (Compared to baseline, median changes in estimated glomerular filtration rate [eGFR] were $-5.0 \mathrm{~mL} / \mathrm{min}$ and $-0.3 \mathrm{~mL} / \mathrm{min}$, respectively; bone mineral density changes were $-3.290 \%$ and $1.233 \%\left[\mathrm{~g} / \mathrm{cm}^{2}\right]$, respectively). ${ }^{224-227}$ Therefore, it is recommended to avoid the use of tenofovir DF among patients with risk factors for renal dysfunc- 
Table 10. Nucleos(t)ide analogue dosage adjustment for adult patients with altered creatinine clearance

\begin{tabular}{|c|c|}
\hline Creatinine clearance $(\mathrm{mL} / \mathrm{min})^{*}$ & Recommended dose \\
\hline \multicolumn{2}{|l|}{ Nucleoside analogues } \\
\hline \multicolumn{2}{|l|}{ Lamivudine } \\
\hline$\geq 50$ & 100 mg q 24 hrs \\
\hline $30-49$ & $100 \mathrm{mg}$ first dose, then $50 \mathrm{mg} \mathrm{q} 24 \mathrm{hrs}$ \\
\hline $15-29$ & $100 \mathrm{mg}$ first dose, then $25 \mathrm{mg} \mathrm{q} 24 \mathrm{hrs}$ \\
\hline $5-14$ & $35 \mathrm{mg}$ first dose, then $15 \mathrm{mg} \mathrm{q} 24 \mathrm{hrs}$ \\
\hline$<5$ & $35 \mathrm{mg}$ first dose, then $10 \mathrm{mg} \mathrm{q} 24 \mathrm{hrs}$ \\
\hline \multicolumn{2}{|l|}{ Telbivudine } \\
\hline$\geq 50$ & $600 \mathrm{mg}$ q $24 \mathrm{hrs}$ \\
\hline $30-49$ & $600 \mathrm{mg} \mathrm{q} 48 \mathrm{hrs}$ \\
\hline$<30$ (not requiring dialysis) & $600 \mathrm{mg} \mathrm{q} 72 \mathrm{hrs}$ \\
\hline End-stage renal disease $^{\dagger}$ & $600 \mathrm{mg} \mathrm{q} 96 \mathrm{hrs}$ \\
\hline \multicolumn{2}{|l|}{ Entecavir } \\
\hline \multicolumn{2}{|l|}{ Entecavir 0.5 mg (NA naïve) } \\
\hline$\geq 50$ & $0.5 \mathrm{mg} \mathrm{q} 24 \mathrm{hrs}$ \\
\hline $30-49$ & $0.25 \mathrm{mg} \mathrm{q} 24 \mathrm{hrs}$ or $0.5 \mathrm{mg} \mathrm{q} 48 \mathrm{hrs}$ \\
\hline $10-29$ & 0.15 mg q 24 hrs or 0.5 mg q 72 hrs \\
\hline$<10$ or hemodialysis ${ }^{\dagger}$ or continuous ambulatory peritoneal dialysis & $0.05 \mathrm{mg} \mathrm{q} 24$ hrs or $0.5 \mathrm{mg} \mathrm{q} 7$ days \\
\hline \multicolumn{2}{|l|}{ Entecavir 1 mg (Lamivudine refractory/resistant) } \\
\hline$\geq 50$ & $1 \mathrm{mg} \mathrm{q} 24 \mathrm{hrs}$ \\
\hline $30-49$ & $0.5 \mathrm{mg} \mathrm{q} 24 \mathrm{hrs}$ or $1 \mathrm{mg} \mathrm{q} 48$ hrs \\
\hline $10-29$ & $0.3 \mathrm{mg} \mathrm{q} 24 \mathrm{hrs}$ or $1 \mathrm{mg} \mathrm{q} 72 \mathrm{hrs}$ \\
\hline$<10$ or hemodialysis ${ }^{\dagger}$ or continuous ambulatory peritoneal dialysis & 0.1 mg q 24 hrs or 1 mg q 7 days \\
\hline \multicolumn{2}{|l|}{ Nucleotide analogues } \\
\hline \multicolumn{2}{|l|}{ Adefovir } \\
\hline$\geq 50$ & $10 \mathrm{mg} \mathrm{q} 24 \mathrm{hrs}$ \\
\hline $20-49$ & $10 \mathrm{mg} \mathrm{q} 48 \mathrm{hrs}$ \\
\hline $10-19$ & $10 \mathrm{mg} \mathrm{q} 72 \mathrm{hrs}$ \\
\hline$<10$ & No recommendation \\
\hline Hemodialysis $^{\dagger}$ & 10 mg q 7 days following dialysis \\
\hline \multicolumn{2}{|l|}{ Besifovir } \\
\hline$\geq 50$ & 150 mg q 24 hrs \\
\hline$<50$ & No recommendation \\
\hline \multicolumn{2}{|l|}{ Tenofovir dipivoxil fumarate } \\
\hline$\geq 50$ & 300 mg q 24 hrs \\
\hline $30-49$ & 300 mg q 48 hrs \\
\hline $10-29$ & 300 mg q 72-96 hrs \\
\hline$<10$ & No recommendation \\
\hline$<10$ with hemodialysis ${ }^{\ddagger}$ & 300 mg q 7 days or after a total of approximately 12 hrs of dialysis \\
\hline \multicolumn{2}{|l|}{ Tenofovir alafenamide fumarate } \\
\hline$\geq 15$ & $25 \mathrm{mg} \mathrm{q} 24 \mathrm{hrs}$ \\
\hline$<15$ & No recommendation \\
\hline
\end{tabular}

NA, nulcleos(t)ide analogue.

${ }^{*}$ Calculated using ideal (lean) body weight.

${ }^{\dagger}$ Administer after hemodialysis.

${ }^{\ddagger}$ Generally once a weekly assuming three hemodialysis sessions a week of approximately 4 hours duration. Administer following completion of dialysis. 
tion such as baseline eGFR $<60 \mathrm{~mL} / \mathrm{min}$, proteinuria, albuminuria (urine albumin: creatinine ratio $>30 \mathrm{mg} / \mathrm{g}$ ), hypophosphatemia $(<2.5 \mathrm{mg} / \mathrm{dL})$, uncontrolled diabetes, or hypertension. If patients have a diagnosis of osteopenia or osteoporosis, need to be on chronic steroids treatment, or take other medications that may lower the bone density, other antivirals that affect bone density less should be considered over tenofovir DF (Fig. 4). ${ }^{97}$

In addition to tenofovir $A F$, entecavir and besifovir have less of an effect on renal function and bone metabolism. In a retrospective study comparing tenofovir DF and entecavir, the mean eGFR percentage decline of was significant in patients with tenofovir DF at week 48,96 , and 144 . Also, bone density decline in the lumbar spine and hip was greater in patients with tenofovir DF than those with entecavir. In addition, osteopenia or osteoporosis prevalence ( $T$ score $<-1.0$ ) was much higher in the tenofovir DF group than the entecavir group at week 48,96 , and 144 . Multivariate analysis showed the predictive factor for bone loss at week 144 was tenofovir DF use. ${ }^{397}$

Besifovir, recently approved for use, had been evaluated in a clinical trial for safety in reduction of renal function and bone density. In a Phase $2 b$ clinical trial, compared to entecavir, patients on besifovir didn't have serum creatinine level increase by more than $0.5 \mathrm{mg} / \mathrm{dL}$ or a significant decrease in eGFR for a 48week period. ${ }^{398}$ During the two-year extended study period, grade 1 elevation of creatinine level (defined as increase of more than $>0.3 \mathrm{mg} / \mathrm{dL}$ or $1.5-2.0$ times the baseline creatinine) was observed in $35.5 \%, 17.9 \%$, and $53.5 \%$ of the group receiving besifovir $90 \mathrm{mg}, 150 \mathrm{mg}$, and $0.5 \mathrm{mg}$ of entecavir, respectively. Hypophosphatemia was observed in $12.9 \%, 10.7 \%$, and $10.0 \%$ of the same group, respectively, but there was no statistical difference among the three groups, and there was no treatment discontinuation or patients who developed symptoms of hypophosphatemia. ${ }^{335}$ Therefore, besifovir is thought to have similar effect on renal function or serum phosphate level compared to entecavir.

\section{Patients who developed renal dysfunction or decrease in bone density on treatment with NAs}

If patient develop renal dysfunction or decrease in bone density while on NAs, one needs to find the causative factors to correct them and adjust the dose modification accordingly (Table 10). Or, there needs to be a review of the need for drug change (Fig. 4).

In a Phase 3 clinical trial comparing tenofovir AF and tenofovir $D F$, patients were given either treatment for 96 weeks each, and afterwards, all received tenofovir AF up until 144 weeks. During

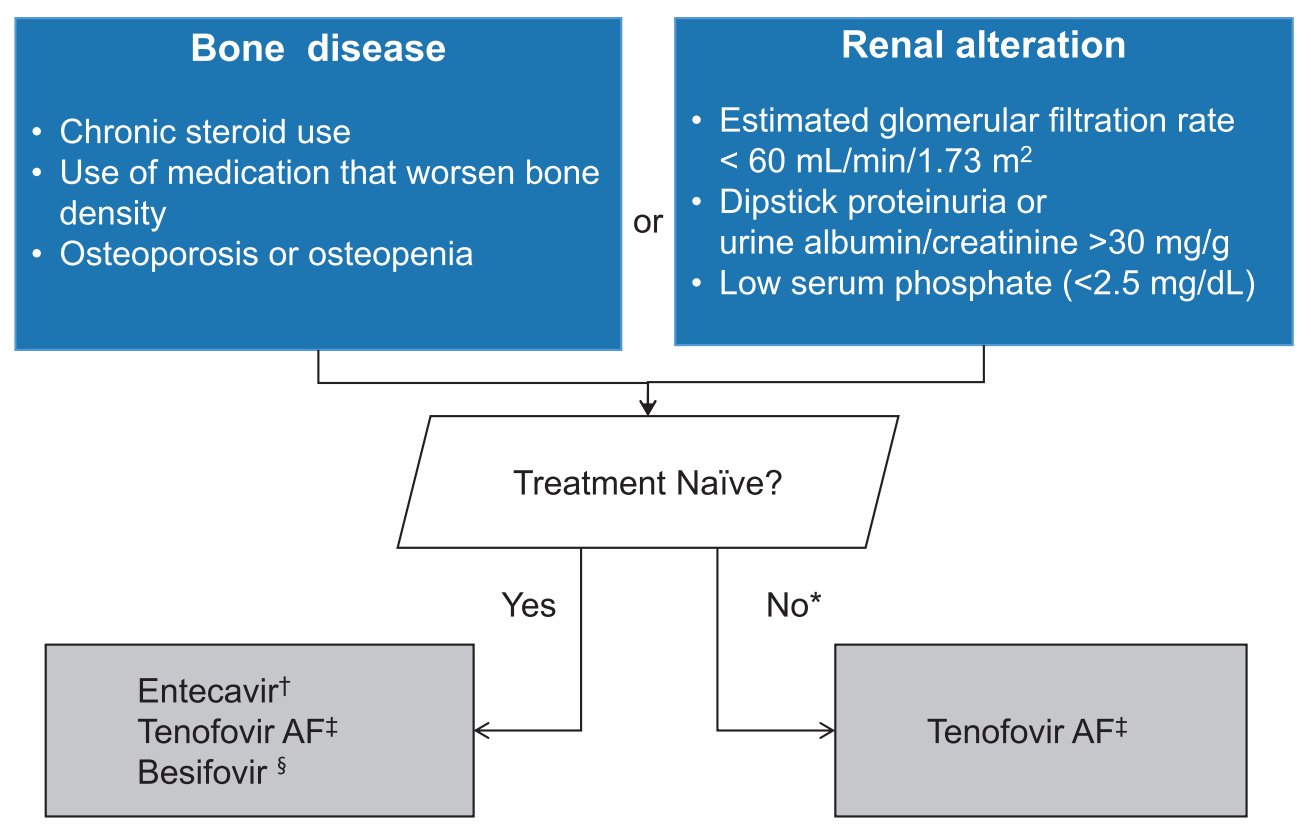

Figure 4. Indications for selecting entecavir, tenofovir alafenamide fumarate, or besifovir over tenofovir disoproxil fumarate. Concomitant comorbidities and past history of antiviral treatment should be considered for choosing nculeos(t)ide analogues (modified from European Association for the Study of the Liver ${ }^{97}$ ). Tenofovir AF, tenofovir alafenamide fumarate. "In case of history of antiviral resistance, refer to Table 9; ${ }^{\dagger}$ Entecavir needs dose adjustments if creatinine clearance $<50 \mathrm{~mL} / \mathrm{min}$, refer to Table 10 ; ${ }^{*}$ Not indicated if creatinine clearance $<15 \mathrm{~mL} / \mathrm{min}$; ${ }^{5}$ Not indicated if creatinine clearance $<50 \mathrm{~mL} / \mathrm{min}$. 
the first 96 weeks, regardless of presence of risk factors, renal function and bone density worsened among patients receiving tenofovir DF. After the antiviral was switched to tenofovir AF until the 144th week, both renal function and bone density improved. ${ }^{224-226,399}$ In patients receiving tenofovir DF, eGFR decreased by $-4.6 \mathrm{~mL} / \mathrm{min}$ compared to the baseline at the 96th week. After switching to tenofovir $A F$, the eGFR reduction was $0.06 \mathrm{~mL} / \mathrm{min}$ compared to the baseline at the 144th week, showing no significant difference between the two groups. ${ }^{224-227}$ Patients receiving tenofovir DF showed greater reduction in spine and hip density during the 96-week compared to those receiving tenofovir AF. After switching to tenofovir AF, they showed significant improvement in bone density score at the 144th week compared to the score at the 96th week. ${ }^{399}$ Therefore, it is thought that the reduction in renal function and/or bone density while on tenofovir DF may be improved by switching to tenofovir AF.

In a Phase 3 trial comparing besifovir with tenofovir DF for 48 weeks, patients receiving besifovir $150 \mathrm{mg}$ had smaller reduction in the eGFR compared to those receiving tenofovir DF $(-1.7 \mathrm{~mL} / \mathrm{min}$ and $-7.8 \mathrm{~mL} / \mathrm{min}$, respectively). After switching from tenofovir DF to besifovir and re-evaluated at the 96th week, the eGFR recovered close to the baseline. Therefore, the decrease in renal function while on tenofovir DF may be improved after switching to besifovir. In addition, specifically for bone density change, patients receiving besifovir $150 \mathrm{mg}$ had a small reduction of the T-score change (reflective of bone density) of $-0.02 \pm 0.44$ at the end of the 48th week. Those receiving tenofovir DF had higher reduction of $-0.09 \pm 0.87$. Besifovir had significantly less effect on the bone density. In particular, after switching from tenofovir DF to besifovir, T-score reduction changed from $-0.09 \pm 0.87$ to $-0.0 \pm 0.59$, showing an improvement in the bone mineral density. ${ }^{334}$

Therefore, during the NA treatment of $\mathrm{CHB}$, if patients develop renal dysfunction or metabolic bone disease, and/or carry risk factors, appropriate drug change can be an option for overcoming the side effects of the NAs (Fig. 4).

\section{[Recommendations]}

1. Entecavir, tenofovir $A F$, and besifovir are preferred over tenofovir DF in treatment-naïve CHB patients with or at risk of renal dysfunction or metabolic bone disease. (B1)

2. Treatment can be switched to tenofovir AF, besifovir, or entecavir depending on treatment history, in patients on tenofovir DF with or at risk of renal dysfunction or metabolic bone disease. (B1)

3. NA doses should be adequately adjusted for creatinine clearance.
Tenofovir AF is not recommended for $\mathrm{CHB}$ patients with creatinine clearance $<15 \mathrm{~mL} / \mathrm{min}$. Besifovir is not recommended for those with creatinine clearance $<50 \mathrm{~mL} / \mathrm{min}$, and tenofovir DF is not recommended for those with creatinine clearance $<10 \mathrm{~mL} / \mathrm{min}$ without renal replacement therapy. (B1)

\section{Patients with acute hepatitis B}

It is well known that more than $95 \%$ of adult patients with acute hepatitis $B$ infection clear the virus without antiviral therapy and do not progress to chronic illness, but some patients go onto develop severe hepatitis. ${ }^{400,401}$ Severe acute hepatitis B can be defined as having an international normalized ratio (INR) $>1.5$, severe jaundice, or progression to hepatic failure. ${ }^{402,403}$

Regarding the need to antiviral therapy for acute hepatitis $B$, in the past, there were studies that suggested antiviral therapy could interfere with body's natural immune response, prevent developing virus-specific neutralizing antibodies, and increase the risk of progression to $\mathrm{CHB}^{404}$ In a meta-analysis of 7 studies, involving 597 patients who received antiviral treatment for acute hepatitis $B$, those receiving lamivudine compared to placebo had higher risk of progression to $\mathrm{CHB}(\mathrm{OR}, 1.99 ; 95 \% \mathrm{Cl}, 1.05-3.77) .{ }^{405} \mathrm{~A}$ randomized controlled trial of 71 patients with severe acute hepatitis $B$ showed that those receiving lamivudine $(n=31)$ had significantly lower serum HBV DNA after 4 weeks $\left(3.7 \log _{10}\right.$ copies $/ \mathrm{mL}$ ) compared to those receiving placebo ( $n=40 ; 4.2 \log _{10}$ copies $/ \mathrm{mL}$ ). After 12 months, there was no significant difference in the HBsAg-negative rates between the two groups (93.5\% vs. 96.7\%, respectively). Additionally, after 1 year, anti-HBs seropositive rate for those receiving lamivudine was $67.7 \%$, lower than $85 \%$ for those receiving placebo, but there was no statistically significant difference between the two. ${ }^{406}$ In another randomized controlled trial involving 80 patients, comparing lamivudine $(n=40)$ and placebo $(n=40)$, the anti-HBs seropositive rate was significantly lower for those receiving lamivudine at $62.5 \%$ compared to those receiving placebo (85\%). However, those receiving lamivudine had significant improvement in blood levels such as coagulopathy or jaundice, and significantly lower mortality rate than those receiving placebo ( $7.5 \%$ vs. $25 \%$, respectively). ${ }^{407}$

There is insufficient evidence from randomized controlled trials for early antiviral therapy in hepatitis B infection. In one cohort study, on the other hand, early administration of a potent antiviral medication was associated with prevention of acute hepatic failure as well as lower rate of liver transplantation ultimately and improved survival. ${ }^{402,408}$ 
For the treatment of acute hepatitis B, lamivudine has been widely used based on results from several well-designed controlled trials. ${ }^{406,407,409,410}$ In a study of entecavir comparing patients who received lamivudine $(n=69)$ and entecavir $(n=21)$, HBsAg seroconversion rates after 24 weeks were $23.18 \%$ for the lamivudine group and $52.38 \%$ for the entecavir group. ${ }^{411}$ There is a case report of using tenofovir DF as a treatment for acute hepatitis $B$. $^{412}$

\section{[Recommendations]}

1. NAs can be initiated in patients with severe acute hepatitis $B$ (e.g., coagulopathy, severe jaundice, liver failure). (B1)

\section{Patients on immunosuppression or chemotherapy}

The progression of $\mathrm{CHB}$ is determined by the interaction between the virus and host immune response. Therefore, if the immune response is suppressed by immunosuppressive therapy or anticancer chemotherapy, the risk of reactivation increases. ${ }^{413}$

\section{Reactivation of $\mathrm{CHB}$}

Reactivation of hepatitis $B$ indicates to the recurrence of active necrotizing inflammatory disease in patients in inactive phase of $\mathrm{CHB}$ or those recovered from previous active infection. It can be largely divided into two categories, "exacerbation of chronic HBV infection" for those with positive HBsAg and "relapse of past HBV infection" for those with negative $\mathrm{HBsAg}$ and positive anti-HBc. ${ }^{414}$ In the latter category, patients who remained in an "occult HBV infection" status may show viral replication triggered by immunosuppression, leading to reverse seroconversion or seroreversion, with redetection of HBsAg. ${ }^{415-418}$ The exacerbation of chronic HBV infection is defined in those with seropositive HBsAg as an increase of serum HBV DNA by more than 100 times the baseline level. The relapse of past HBV infection is defined as seroconversion of HBsAg negative to positive, or detection of serum HBV DNA from none to positive. Hepatitis flare is defined as serum ALT level more than 3 times the baseline level or increase by more than $100 \mathrm{IU} / \mathrm{L}^{419,420}$

Various rates of reactivation had been reported, but it is generally known to be about $20-50 \%$. For an accurate diagnosis, liver damage related to chemotherapy, tumor metastasis, or hepatitis secondary to other viruses should be excluded. In many cases, patients are asymptomatic but occasionally present with jaundice, or are found in various stages such as decompensated liver diseases or even death. ${ }^{418,421-423}$ Typical reactivation is seen by detection of serum HBV DNA during immunosuppression or chemotherapy, or elevation of serum ALT after stopping the immunosuppressive therapy. If the reactivation occurs during chemotherapy, it can lead to reduction or discontinuation of chemotherapy, adversely affecting the success of the chemotherapy. ${ }^{424-426}$ There are risk factors, related to the virus, the host, and treatment, which are predictive of hepatitis $B$ reactivation: virus factors include serum HBV DNA, HBeAg seropositivity, hepatocyte cccDNA, and PC/BCP mutation prior to the treatment; host factors include types of malignant tumors, male gender, young age, and high serum ALT levels; and, treatment factors include the type and intensity of immunosuppressant or chemotherapy regimen, HSCT, and/or solid organ transplantation. ${ }^{427}$ The type and intensity of chemotherapy regimen related to the risk of hepatitis $B$ reactivation can be classified into three categories: high risk group (reactivation risk of $10 \%$ or more), moderate risk group (reactivation risk between 1-10\%) and low risk group (reactivation risk below 10\%) (Table 11). ${ }^{387,428,429}$

Reactivation of hepatitis B during chemotherapy for hematologic malignancy: During the chemotherapy for lymphoma, hepatitis $B$ reactivation is reported to be frequent with the rate up to $24-67 \%$. It not only implies that the chemotherapy used for lymphoma is strong enough to cause bone marrow suppression, but also that patients with lymphoma have higher rates of seropositive HBsAg than those without lymphoma. ${ }^{422,430-432}$ Rituximab, commonly used in combination with steroids for the treatment of lymphoma, is known to increase the risk of reactivation. ${ }^{433,434}$ Rituximab therapy increased the risk of hepatitis $B$ reactivation in patients with non-Hodgkin's lymphoma who had seropositive $\mathrm{HBsAg}$ or seronegative $\mathrm{HBsAg} /$ seropositive anti-HBC combination (relative risk [RR], 2.14; 95\% Cl, 1.42-3.22; $P=0.0003$ ). In particular, in patients with seronegative $\mathrm{HBsAg} /$ seropositive anti-HBc combination, the use of rituximab therapy was associated with higher $R R$ of reactivation ( $R R, 5.51){ }^{435}$

There was a significant difference in the reactivation of hepatitis $B$ in patients with and without prophylactic antiviral therapy (13.3\% vs. $60 \%$ ) during treatment with rituximab. ${ }^{436}$ Furthermore, prior to receiving chemotherapy (e.g. rituximab-cyclophosphamide, hydroxydaunorubicin, Oncovin, prednisone [R-CHOP]), screening for hepatitis $B$ in all patients, rather than limiting to high-risk groups, resulted in a 10-fold reduction in hepatitis $B$ reactivation rate and economic and survival benefits. ${ }^{437}$

With other hematologic malignancies, if patients are receiving high-intensity chemotherapy prior to HSCT, the risk of reactivation is high. ${ }^{438,439}$ In particular, in patients with seropositive HBsAg or seronegative $\mathrm{HBsAg} /$ seropositive anti- $\mathrm{HBc}$ awaiting high intensity 
Table 11. Risk of hepatitis B reactivation associated with immunosuppressive therapy

\begin{tabular}{|c|c|}
\hline Risk of reactivation & Immunosuppressive therapy \\
\hline \multicolumn{2}{|l|}{ HBsAg-positive } \\
\hline \multirow[t]{5}{*}{ High risk ( $\geq 10 \%)$} & B-cell depleting agents (rituximab, ofatumumab, natalizumab, alemtuzumab and ibritumomab) \\
\hline & High-dose corticosteroids (prednisone $\geq 20$ mg/day, $\geq 4$-week) \\
\hline & Anthracyclines (doxorubicin and epirubicin) \\
\hline & More potent TNFa inhibitors (infliximab, adalimumab, certolizumab and golimumb) \\
\hline & Local therapy for HCC (transcatheter arterial chemoembolization) \\
\hline \multirow[t]{8}{*}{ Moderate risk (1-10\%) } & Systemic chemotherapy \\
\hline & Moderate-dose corticosteroids (prednisone 10-20 mg/day, $\geq 4$-week) \\
\hline & Less potent TNFa inhibitors (etanercept) \\
\hline & Cytokine-based therapies (abatacept, ustekinumab, mogamulizumab, natalizumab and vedolizumab) \\
\hline & Immunophilin inhibitors (cyclosporine) \\
\hline & Tyrosine-kinase inhibitors (imatinib and nilotinib) \\
\hline & Proteasome inhibitors (bortezomib) \\
\hline & Histone deacetylase inhibitors \\
\hline \multirow[t]{3}{*}{ Low risk $(<1 \%)$} & Antimetabolites, azathioprine, 6-mercaptopurine, methotrexate \\
\hline & Low-dose corticosteroids (prednisone <10 mg/day) \\
\hline & Intra-articular steroid injections (extremely low risk) \\
\hline \multicolumn{2}{|c|}{ HBsAg-negative/anti-HBc positive } \\
\hline High risk ( $\geq 10 \%)$ & B-cell depleting agents (rituximab, ofatumumab, natalizumab, alemtuzumab and ibritumomab) \\
\hline \multirow[t]{9}{*}{ Moderate risk (1-10\%) } & High-dose corticosteroids (prednisone $\geq 20$ mg/day, $\geq 4$-week) \\
\hline & Anthracyclines (doxorubicin and epirubicin) \\
\hline & More potent TNFa inhibitors (infliximab, adalimumab, certolizumab and golimumb) \\
\hline & Systemic chemotherapy \\
\hline & Cytokine-based therapies (abatacept, ustekinumab, mogamulizumab, natalizumab and vedolizumab) \\
\hline & Immunophilin inhibitors (cyclosporine) \\
\hline & Tyrosine-kinase inhibitors (imatinib, nilotinib) \\
\hline & Proteasome inhibitors (bortezomib) \\
\hline & Histone deacetylase inhibitors \\
\hline \multirow[t]{2}{*}{ Low risk $(<1 \%)$} & Moderate-dose (prednisone 10-20 mg/day) and Low-dose (prednisone $<10 \mathrm{mg} /$ day) corticosteroids \\
\hline & Antimetabolite, azathioprine, 6-mercaptopurine, methotrexate \\
\hline
\end{tabular}

Modified from Loomba et al. ${ }^{387}$

HBsAg, hepatitis B surface antigen; TNFa, tumor necrosis factor alfa; HCC, hepatocellular carcinoma; anti-HBc, antibody to hepatitis B core antigen.

chemotherapy prior to HSCT, antiviral therapy with a high barrier to resistance is recommended. ${ }^{440}$ During immunosuppressive therapy or chemotherapy for hematologic disorders, for patients with evidence of hepatitis B infection, prophylactic treatment with lamivudine or entecavir can significantly lower the reactivation rate of hepatitis $B .^{441,442}$

Reactivation of hepatitis B during chemotherapy for solid tumors: Reactivation of hepatitis $B$ in patients with solid tumors is known to be about $14-21 \%$, but for those with breast cancer, it is higher at about $41-70 \%$, which is thought to be related to the high dosages of breast cancer treatment agents as well as the use of anthracycline-based chemotherapy and steroids. ${ }^{426,443,444}$ Steroids not only suppress immune system but directly stimulates the replication of $\mathrm{HBV}$, thus raising the risk of reactivation. It is reported that the use of prophylactic antiviral agents in most solid tumors, such as breast and lung cancers, has 
significantly reduced the rate of hepatitis B reactivation and discontinuation of chemotherapy treatment. ${ }^{445-448}$

Reactivation of hepatitis B during treatment for inflammatory bowel disease (IBD) or rheumatoid arthritis

(RA): The reactivation of hepatitis B may also be associated with the use of TNF a inhibitors (infliximab, etanercept, adalimumab, etc.) for the treatment of IBD or RA. ${ }^{449-451}$ In case of TNF a inhibitors and disease-modifying antirheumatic drugs (DMARDs) used for RA treatment, the rate of reactivation of hepatitis B was reported to be around $12.3 \%$ in patients with seropositive HBsAg. ${ }^{452}$ In another study, the reactivation was reported in 39\% of HBsAg-positive patients and 5\% of anti-HBc-positive patients, and among those given antiviral prophylaxis, the reactivation rate was significantly lower. ${ }^{453}$

Acute exacerbation of hepatitis B while on immune check point inhibitors: Recently, immune checkpoint inhibitors such as anti-PD-1 (nivolumab) and anti-CTLA4 (ipilimumab) therapy have been used in various carcinomas including liver cancer. Although there is a concern about the possibility of acute exacerbation of hepatitis B in relation to these therapeutic agents, there isn't sufficient data yet, and the discussion regarding the consideration of future antiviral prophylaxis is necessary.

\section{Start and end point of prophylactic antiviral treatment}

When the reactivation of hepatitis $B$ occurs, there is a risk of liver failure or even death. Therefore, the prevention is foremost important. Prior to starting an immunosuppressive therapy or chemotherapy, screening for $\mathrm{HBsAg}$ and anti-HBc is necessary. If there is no evidence of $\mathrm{HBV}$ infection in the past (with negative $\mathrm{HBs} A g$ and anti-HBC), HBV vaccination may be considered. In HBsAg positive cases, regardless of serum HBV DNA level, antiviral prophylaxis is recommended. Instead of waiting for the serum HBV DNA level to rise, administering an antiviral agent at the start of the immunosuppressive therapy or chemotherapy, or 7 days prior to the treatment start date, is reported to be more effective. ${ }^{448,454-456}$ The end point of the prophylactic antiviral treatment should theoretically be continued until the immune system is adequately recovered, but there is lack of sufficient evidence to suggest a specific end point. It has been reported that the risk of HBV reactivation is high when prophylactic lamivudine is discontinued about 3 months after the end of chemotherapy. The risk is especially higher when the serum HBV DNA prior to the treatment is elevated $(\geq 2,000 \mathrm{IU} / \mathrm{mL}) .{ }^{457}$ Therefore, when HBV is actively replicating before prophylactic antiviral therapy, following the present CHB treatment guidelines for the discontinuation of anti- viral agents may prevent virus reactivation after treatment. However, regardless of the serum HBV DNA level prior to the treatment, reactivation still is reported more than 6 months after the completion of chemotherapy, so caution is required. Therefore, antiviral prophylaxis should be maintained for at least 6 months minimum after the chemotherapy is completed, and extension should be considered according to the chemotherapy risk. Especially, for patients receiving chemotherapy involving rituximab, it is recommended to extend the antiviral prophylaxis to at least 12 months after the completion of chemotherapy. ${ }^{458-460}$ There is a need to closely monitor for relapse for at least 12 months after the prophylaxis is over.

On the other hand, reactivation of hepatitis B may occur not only when HBsAg is positive, as described above, but also when HBsAg is negative and anti-HBc is positive. Especially, when immunosuppressed, patients with seropositive anti-HBC alone had a higher risk of hepatitis $B$ reactivation than patients with both seropositive anti-HBC and anti-HBs. ${ }^{387,461}$ Thus, when patients, who are $\mathrm{HBSAg}$ negative and anti-HBC positive, receive rituximab-involving therapy or fall into a high-risk group such as being considered for HSCT for leukemia, antiviral prophylaxis should be considered regardless of HBV DNA detection or HBsAg seroconversion. For patients in a moderate or low-risk group of chemotherapy, HBsAg and HBV DNA levels should be periodically monitored (at intervals of 1-3 months) during or after chemotherapy to initiate antiviral treatment when hepatitis $B$ reactivation is detected. ${ }^{387}$

\section{Treatment medications}

Lamivudine is the most widely studied drug for prophylactic antiviral therapy. It is well known to significantly reduce reactivation, liver failure, and death according to randomized controlled trials of lymphoma patients in Hong Kong and Taiwan ${ }^{431,439,454,462}$ However, lamivudine has been reported to be resistant even during prophylaxis. If the treatment duration is expected to be long, it is necessary to select a therapeutic agent with a high barrier to resistance considering the resistance rate. ${ }^{431}$ In a retrospective study of lymphoma patients, the incidence of hepatitis and chemotherapy disruption due to HBV reactivation was significantly lower in the entecavir group than in the lamivudine group. ${ }^{463}$ In a metaanalysis, entecavir prophylaxis was shown to prevent reactivation of hepatitis B more effectively compared to lamivudine prophylaxis. ${ }^{464}$ Tenofovir DF was also reported to be effective for this purpose. In a recent randomized prospective controlled trial, under treatment with rituximab the rate of hepatitis $B$ reactivation was $0 \%$ in the group prophylactically treated with tenofovir DF com- 
pared to $10.7 \%$ in the control group $(P=0.091) .{ }^{438}$ It is necessary to conduct prospective studies on appropriate antiviral agents and treatment duration for various types of cancer, including solid tumors, and chemotherapeutic agents.

\section{[Recommendations]}

1. Screening for $\mathrm{HBsAg}$ and anti-HBc before immunosuppression or chemotherapy is recommended. If either is positive, HBV DNA testing should be performed. (A1)

2. If either HBsAg is positive or HBV DNA is detected, prophylactic antiviral therapy should be initiated before or at the start of immunosuppression or chemotherapy. (A1)

Antiviral agents should be selected based on comprehensive consideration of serum HBV DNA levels, the intensity and duration of immunosuppression or chemotherapy, and the cost. If baseline serum HBV DNA is high or long-term therapy is anticipated, tenofovir or entecavir is preferred. (B1)

3. In HBsAg-negative, HBV DNA-undetectable, anti-HBc-positive patients, serum HBsAg and HBV DNA should be monitored during high-risk immunosuppression/chemotherapy and antiviral therapy started when HBV reactivation occurs. (A1) In particular, when a regimen includes rituximab, antiviral therapy can be initiated promptly at the start of immunosuppression or chemotherapy. (B1)

4. Prophylactic antiviral therapy should be maintained for at least 6 months after the termination of immunosuppression or chemotherapy and for at least 12 months after the termination of therapy if rituximab was included. (B1)

5. Periodic monitoring of serum HBV DNA is recommended during and after prophylactic antiviral therapy. (A1)

\section{Liver transplant patients}

Before the era of prophylactic antivirals, most patients with liver diseases related to hepatitis B experienced severe liver damage and had low survival rates from reactivation of hepatitis B after liver transplantation. ${ }^{465-471}$ After the introduction of HBIG, a large cohort study of $372 \mathrm{HBsAg}$-positive patients reported that patients treated with HBIG for more than 6 months post-transplantation had significantly lower hepatitis B reactivation rates and higher long-term survival rates compared to patients treated for less than 6 months or who never received prophylaxis. ${ }^{472}$ Lamivudine and HBIG combination therapy reduced the reactivation rate of hepatitis B in 1-2 years to less than $10 \%$. From the cost-effective perspective, the combination therapy had better result compared to high-dose HBIG monotherapy $(10,000 \mathrm{IU})$. $^{47-476}$ In a meta-analysis, lamivudine and HBIG combination therapy has shown a 12-fold reduction in the reactivation rates and related mortality rates of hepatitis B virus compared to HBIG alone. ${ }^{477,478}$

\section{HBsAg-positive recipients}

Lamivudine therapy without HBIG was associated with $40 \%$ reactivation rate of hepatitis B 4 years after liver transplantation $^{479,480}$ When adefovir and lamivudine were administered together, no reactivation was noted during the 22-month follow-up period. ${ }^{481}$ In a study using entecavir, during the 26-53 months follow-up period, HBsAg seronegative rate was $88-91 \%$, over $98 \%$ had non-detectable level of HBV DNA, and reactivation rate was lower than when lamivudine alone was used. ${ }^{482,483}$ In patients with non-detectable HBV DNA level at the time of liver transplantation, a study was conducted using a single agent of antiviral prophylaxis without HBIG was attempted. During the 8-year follow-up of 362 patients, HBsAg negative rate was $88 \%$ and hepatitis B reactivation rate was $2 \% .{ }^{482}$ In the same study group, 265 patients were given entecavir only and followed for 59 months, and there was no reactivation of hepatitis $B .{ }^{484}$ However, in a meta-analysis of 519 patients from 17 studies, the combination of lamivudine and HBIG (6.1\%) had a similar effect of suppressing reactivation compared to using entecavir or tenofovir DF alone ( $3.9 \%, P=0.51)$, but had a higher rate of reactivation compared to the combination therapy of entecavir or tenofovir DF with HBIG $(1 \%, P<0.001){ }^{485}$ Therefore, to prevent reactivation after the transplantation, a potent antiviral agent combined with HBIG is recommended.

On the other hand, to reduce the use of expensive $\mathrm{HBIG}$, there have been studies that use a small amount of HBIG or HBIG combined with an antiviral agent for a short term and then switched to an antiviral agent alone. Gane et al. reported in a study of 147 liver transplant patients, lamivudine combined with low dose HBIG (400-800 IU) resulted in 4\% reactivation rate of hepatitis B over 5 years. ${ }^{486}$ In another case where lamivudine and adefovir were used in combination with low-dose HBIG (400-800 IU) only at the beginning of transplantation and then switched to lamivudine and adefovir only, no reactivation was reported during the 57-month follow-up period. ${ }^{481}$ When HBIG was used in combination initially, and then switched to entecavir or tenofovir DF alone, no reactivation was reported afterward. ${ }^{487,488}$ Recently, based on the results of these studies, it is recommended to adjust the dose and the duration of HBIG use by assessing the hepatitis B reactivation risk of an individual patient at the time of the transplantation. One may consider using HBIG for a short duration in patients with non-detectable HBV DNA level at the time of transplantation. ${ }^{489-491}$ Conversely, patients with a high risk of hepatitis $B$ reactivation (e.g. Patients with detectable HBV DNA level, positive HBeAg, HCC, or HDV or HIV co-infection at the time of transplan- 
tation, or those with anticipated poor compliance with antiviral medications) may require a lifelong use of HBIG and anti-viral agent. ${ }^{492,493}$

\section{HBsAg-negative/anti-HBc-positive donors}

If HBsAg-negative patients receive liver transplantation from HBsAg-negative/anti-HBc-positive donors, about $75 \%$ of the recipients are known to newly develop hepatitis B infection. It is reported to be dependent on the recipient's HBV immunization status. Especially when the recipient is anti-HBs negative, the risk of new hepatitis B infection becomes higher. ${ }^{494-496}$ After HBsAg-negative patients receive liver transplantation from HBsAg-negative/antiHBC-positive donors, when HBIG alone was used, more than $20 \%$ newly developed hepatitis B but when lamivudine alone was used, only 2-3\% went onto develop hepatitis B. However, lamivudine and HBIG combination therapy did not show any additional preventive effect compared to lamivudine alone. ${ }^{494,497,498}$ Therefore, if HBsAg-negative patient receive liver transplantation from HBsAg-negative/anti-HBc-positive donors, NA monotherapy is recommended to prevent new hepatitis B infection after transplantation. ${ }^{499}$ However, in a retrospective study of 14 patients receiving liver transplantations from HBsAg-nega tive/anti-HBc-positive donors in Korea, 11 patients receiving HBIG monotherapy had $0 \%$ of new hepatitis B infection. ${ }^{500}$ In a small retrospective study in Japan, HBIG monotherapy had $0 \%$ of recurrence rate of hepatitis $B .^{501}$ Thus, in clinical practice, HBIG monotherapy may also be considered. ${ }^{500,501}$

\section{Treatment medications}

In selecting an antiviral agent, lamivudine was shown to be cost-effective in a cost-effectiveness analysis using the Markov model. ${ }^{502}$ In a study of NA monotherapy, including entecavir and tenofovir $D F$, the recurrence rate with these two agents were similar to lamivudine. ${ }^{502}$ However, when lamivudine was used longterm after liver transplantation, over $50 \%$ had reported lamivudine resistance in 3 years. ${ }^{503-505}$ This lamivudine resistance has negative effects, known to induce inflammatory changes and liver fibrosis between the grafts, or, in severe incidences, to cause death from the liver failure. ${ }^{504,506,507}$ Therefore, as long-term antiviral therapy may be required, the use of antiviral agents with low resistance, such as entecavir and tenofovir, is recommended.

\section{[Recommendations]}

1. In hepatitis B-related liver transplant recipients, a combination of lifelong NAs and HBIG is recommended to prevent recurrence of hepatitis $B$ after liver transplantation. (B1)

However, in the case of HBV DNA not detected before transplantation, modification of HBIG dose and duration may be considered after assessment of risk factors. (B2)

2. After liver transplantation, entecavir or tenofovir DF is recommended. (B1)

In cases of drug resistance, refer to the antiviral resistance chapter. (B1)

3. If the recipient is $\mathrm{HBs} A g$ negative and the donor is anti-HBC positive, administration of NAs is recommended after liver transplantation. (B1)

If NAs cannot be administered, HBIG monotherapy may be considered. (B2)

\section{Non-liver organ transplant recipients}

\section{Non-liver solid organ transplantation}

HBsAg-positive renal transplant recipients are at high risk for persistent viral activity or reactivation and have a significantly higher mortality rate due to liver-related complications such as liver cirrhosis and $\mathrm{HCC} .{ }^{508}$ Recent reports indicate that antiviral therapy increases the survival of HBsAg-positive renal transplant recipients. ${ }^{509,510}$ Lamivudine therapy improves the survival of renal transplant recipients, but in cases of long-term administration, the drug resistance rate is $62 \%$ at 4 years. ${ }^{511}$ Entecavir improves virological response, graft survival, and overall survival compared to lamivudine. ${ }^{512,513}$

Small studies of HBsAg-positive heart transplant recipients have also demonstrated the safety and efficacy of NAs, and the use of entecavir or tenofovir DF is recommended to avoid liver failure due to drug resistance. ${ }^{514,515}$

The risk of HBsAg reversion is low in recipients who are $\mathrm{HBs} A g$ negative and anti-HBC positive. In a Korean cohort study of 951 recipients of kidney transplantation recipients with seronegative $\mathrm{HBsAg} /$ seropositive anti-HBC, the $\mathrm{HBsAg}$ reversion rate was $5.6 \%$ for anti-HBs negative patients, but there was no difference between anti-HBs-positive and anti-HBc-negative recipients. ${ }^{516} \mathrm{How}$ ever, because HBsAg reversion followed by liver failure was reported in recipients with seronegative $\mathrm{HBs} A \mathrm{~g} /$ seropositive anti$H B C$, hepatitis $B$ reactivation should be monitored regularly. In patients who are $A B O$-incompatible and highly sensitized, rituximab is commonly used prior to renal transplantation. However, it can lead to liver failure in those with past HBV infection due to HBsAg reversion or HBV DNA redetection, albeit this risk is very low at low rituximab doses $(200 \mathrm{mg}) .{ }^{516-518}$

In recipients without prior HBV infection who receive a graft 
from an HBsAg-negative/anti-HBc-positive donor, the HBsAg positivity rate and anti- $\mathrm{HBC}$ detection rate are $0.3 \%$ and $3.2 \%$, respectively. ${ }^{519}$ However, in other studies, the HBsAg positivity rate of recipients of a transplanted kidney, heart, lung, or other organ from an HBsAg-negative/anti-HBc-positive donor did not differ from the rate for recipients receiving organs from anti-HBc-negative donors. ${ }^{520,521}$ More studies are needed to determine whether either prophylactic antiviral treatment or HBIG reduces hepatitis B reactivation or HBV transmission in recipients of organs transplanted from a donor with past HBV infection.

\section{Hematopoietic stem cell transplant}

Patients with CHB who require HSCT for hematologic malignancies are immunosuppressed for a prolonged period due to the high-dose chemotherapy and hematological diseases itself. This increases the risk of hepatitis $B$ reactivation and leads to a poor prognosis. $^{522,523}$ In small retrospective studies of $\mathrm{HBsAg-positive}$ recipients of allogeneic or autologous stem cell transplantation, prophylactic lamivudine treatment for 6-12 months significantly reduced the frequency of hepatitis $B$ reactivation (5-10\% vs. $45-$ $50 \%) .{ }^{439}$ In another study, HBsAg-positive recipients of allogeneic stem cell transplants underwent prophylactic antiviral treatment for up to 6 months after termination of immunosuppressive therapy and were followed for 24 months after transplantation. The cumulative reactivation rate of hepatitis $B$ was significantly higher in patients receiving lamivudine (24\%) compared to patients receiving entecavir (2\%). Recent meta-analyses have also demonstrated the efficacy of entecavir in preventing hepatitis $B$ reactivation. $^{524,525}$

Hepatitis $B$ reactivation is not infrequent in HSCT recipients with seronegative $\mathrm{HBsAg} /$ seropositive anti-HBc. In a prospective cohort study in which $62 \mathrm{HBsAg-negative} \mathrm{and} \mathrm{anti-HBc-positive} \mathrm{al-}$ logeneic stem cell transplant recipients were followed for 48 weeks, the 2-year cumulative reactivation rate (detectable HBV DNA $>10 \mathrm{IU} / \mathrm{mL}$ ) was $40.8 \%{ }^{526}$

\section{[Recommendations]}

1. All HBsAg-positive solid organ transplant recipients and HSCT recipients should start prophylactic antiviral treatment at the time of transplantation. (A1)

Entecavir or tenofovir DF is preferred for long-term treatment. (B1)

2. HBsAg-negative, HBV DNA undetectable, but anti-HBcpositive solid organ transplant recipients need regular follow-up to monitor the reactivation of hepatitis B. (B1) 3. HBsAg-negative, HBV DNA undetectable, but anti-HBc positive
HSCT recipients are recommended to start prophylactic antiviral treatment at the time of transplantation. (B1)

\section{Coinfection with other viruses}

\section{HCV coinfection}

In patients with $\mathrm{CHB}$, the anti-HCV positivity rate varies from $1.5 \%$ to $2.37 \%$ in Korea. ${ }^{527,528}$ Patients with HBV/HCV coinfection have more severe necroinflammation and fibrosis than those with a monoinfection, as well as a high risk of cirrhosis and $\mathrm{HCC}^{529,530}$ When introducing direct-acting agents (DAA) in HBV/HCV coinfection, serum HBV DNA and HCV RNA levels should be analyzed to evaluate the replicative status of each virus. If the patient is HCV RNA positive, they should be treated as for HCV monoinfection. If the patient meets the criteria for antiviral treatment for $\mathrm{CHB}$, proper treatment should be started promptly. Hepatitis B reactivation is possible during or after HCV treatment. One metaanalysis showed that detectible or increased levels of HBV DNA were reported in $14.1 \%$ of patients at $4-12$ weeks after DAA treatment, and the incidence of elevated ALT (active hepatitis) was $12.2 \%{ }^{531}$ In a prospective study of patients in Taiwan who were treated with ledipasvir/sofosbuvir, the HBV DNA detection rate was $83 \%$, the HBV DNA 10 -fold increase rate was $53 \%$, and the active hepatitis rate was $6.3 \%{ }^{532}$ According to the US Food and Drug Administration (FDA) Adverse Event Reporting System, two cases resulted in death, and 1 case resulted in liver transplantation. Therefore, serological tests, such as HBsAg and HBV DNA, are recommended before and during DAA therapy. Antiviral treatment for HBV should be considered if there is a marked HBV DNA elevation during DAA therapy. ${ }^{533}$ Entecavir and tenofovir DF are recommended for the treatment of $\mathrm{CHB}$ in patients with $\mathrm{HCV}$ coinfection, but renal function monitoring is warranted if ledipasvir is used with tenofovir DF because it can increase the renal toxicity of tenofovir DF. ${ }^{534}$ The drug interactions between HBV and HCV antiviral agents are summarized in the 2017 KASL Clinical Practice Guidelines for Management of Hepatitis C.

\section{[Recommendations]}

1. CHB patients with HCV co-infection can undergo either treatment according to each treatment strategy. (B1)

2. HBV DNA levels may be elevated during or after treatment of chronic hepatitis $C$, which requires careful monitoring. (B1) 


\section{HIV coinfection}

The incidences of cirrhosis and HCC are higher in patients with HBV/HIV coinfection than in those with HBV monoinfection. ${ }^{535}$ The rate of HBV superinfection among Korean HIV patients is estimated to be approximately $5 \% .{ }^{536}$ With the changes in treatment strategies for HIV infection, the initiation of highly active antiretroviral therapy (HAART) has recently been recommended regardless of the number of $C D 4+T$ cells. Therefore, simultaneous treatment for each virus is recommended for HIV and HBV coinfection. Therapeutic agents should be selected from the tenofovir-based antiretroviral regimen, and combination therapy with emtricitabine or lamivudine, which can inhibit the replication of both viruses, is recommended. ${ }^{537}$ When HAART regimens are changed, antiviral agents that are effective against HBV should be included to avoid HBV reactivation, except in patients who meet the criteria for cessation of antiviral treatment for HBV.

\section{[Recommendations]}

1. In CHB patients with HIV co-infection, tenofovir should be included in HAART. (A1)

\section{HDV coinfection}

It is estimated that $\sim 20$ million people are infected with HDV worldwide, and HDV infections are prevalent in Mediterranean countries, the Middle East, central Africa, and South America. ${ }^{538}$ In one Korean study, the HDV coinfection rate was $0.3 \%$ in 940 patients with $\mathrm{CHB}$, including 75 patients with $\mathrm{HCC}$. In another study of 194 patients that included 64 CHB patients and $130 \mathrm{HCC}$ patients, the rate was $3.6 \% .{ }^{539,540}$ The incidences of cirrhosis and HCC are higher in patients with HBV/HDV coinfection than in those with HBV monoinfection. ${ }^{541,542}$ HDV infection can be diagnosed by detecting anti-HDV or HDV RNA in the serum or by detecting HDV antigens in liver tissues using immunohistochemistry.

The treatment goals are to inhibit HDV replication, normalize ALT levels, and improve histology findings. If the HBV/HDV coinfection patients meet the criteria for antiviral treatment for CHB, oral administration of CHB treatment is necessary to prevent the progression of liver disease. However, NAs for CHB are not recommended for the treatment of HDV infection in patients with HDV superinfection because they cannot inhibit HDV replication. Peginterferon alfa therapy was superior to high-dose interferon alfa therapy and that a combination therapy using an NAs and peginterferon alfa did not improve virological response compared to peginterferon alfa therapy alone. ${ }^{543}$ The sustained virological response at 24 weeks after 48 week of peginterferon alfa therapy is $23-28 \%$, and a sustained virological response can be predicted 24 weeks after the initiation of treatment by measuring serum HDV RNA levels. ${ }^{54-546}$ However, relapse is frequent during longterm follow-ups, as seen in one study with an average follow-up of 4.3 years where sustained virological response was maintained at only $12 \% .{ }^{577}$ In a small study using extended peginterferon alfa therapy for 24 months, $47 \%$ of patients achieved sustained virological response during an average follow-up of 19.5 months after treatment, but further studies are needed. ${ }^{548}$

\section{[Recommendations]}

1. CHB patients with HDV co-infection are recommended to be treated with peginterferon alfa for at least 1 year. (A1)

2. In CHB patients with HDV co-infection, initiation of NAs for $\mathrm{CHB}$ is recommended to prevent the progression of liver disease if either the indications for CHB treatment are met or liver cirrhosis is present. (B1)

\section{Pregnant women and women preparing for pregnancy}

Treatment in pregnant women and women preparing for pregnancy

Immunological changes during pregnancy: Pregnant women with chronic HBV infections are usually in the immune tolerance phase, and changes in the maternal immune system during pregnancy, such as a shift in the Th1-Th2 balance toward a Th2 response, lead to an increase in HBV DNA levels and a reduction in ALT levels. ${ }^{549,550}$ These immune responses are restored after delivery causing a reduction in HBV DNA levels and ALT elevation, and therefore careful monitoring is needed. $.51,552$

Antiviral treatment: The optimal antiviral treatment strategy during pregnancy is based on the general principles for the treatment of CHB. However, all decisions regarding the timing and duration of treatment in pregnancy should include an analysis of the risks and benefits for both the mother and the fetus.

Peginterferon alfa preparations are preferred for patients who are planning a pregnancy as the period of treatment is more clearly defined. However, peginterferon alfa may cause fetal malformations; thus, it is contraindicated during pregnancy. Contraception must be emphasized during therapy and until 6 months after the cessation of therapy.

NAs may cause mitochondrial toxicity by inhibiting mitochondrial DNA replication. ${ }^{553}$ The safety data of various NAs during preg- 
nancy can be found at the Antiretroviral Pregnancy Registry (APR; http://www.apregistry.com). According to the APR, the rates of birth defects among the babies exposed to lamivudine or tenofovir DF in the first trimester (3.1\% and $2.4 \%$ of live births, respectively) are similar to that in the general population $(2.7 \%)$ as reported by the Center for Disease Control and Prevention birth defect surveillance system. Recently, further studies on the efficacy and safety of NAs have been carried out in pregnant women and infants. A small-scale prospective study of Chinese infants of patients who received telbivudine during pregnancy reported that mental development index and psychomotor developmental index at 12-24 months of age were significantly lower than those of the control group. ${ }^{554}$ These results suggest that prenatal telbivudine exposure may lead to motor development delay in offspring. Meanwhile, infants exposed to tenofovir DF did not differ from those who were not exposed in terms of preterm birth, fetal anomalies, and poor development at birth and up to 6-12 months postnatally. In a large cohort study on tenofovir DF-based antiretroviral therapy to prevent MTCT of HIV infection in South Africa and the United States, there was no difference in the growth rate or the standard growth curve at 12 to 24 months after birth, regardless of the duration of antiretroviral therapy exposure or the duration of breastfeeding. ${ }^{555}$ Based on the results of these clinical studies, tenofovir DF is preferred for treatment in pregnant women or patients preparing for pregnancy. Tenofovir AF requires further study.

Maintaining or altering the use of NAs: The decision as to whether to discontinue treatment with NAs in pregnant patients should be individualized. One retrospective study showed that $14 \%$ of pregnant patients with active CHB who did not receive antiviral therapy progressed to hepatic failure or maternal or fetal death. Another retrospective study reported moderate ALT elevations in 16\% of pregnant patients who discontinued NAs pre-pregnancy and in $29-31 \%$ of pregnant and postpartum patients who discontinued NAs in the first trimester. ${ }^{56,557}$ Therefore, if there is a high risk of complications in pregnant women and fetuses due to liver failure, appropriate maintenance of NAs should be considered. Taken together, if patients needed to maintain NAs, tenofovir DF can be continued in patients already taking tenofovir DF. The other agents should be switched to tenofovir DF in preparation for pregnancy or when a pregnancy is detected.

\section{Prevention of MTCT of HBV with NAs}

Influence of breastfeeding on infants: Several studies show the effects of breastfeeding on MTCT in HBsAg-positive pregnant women without antiviral treatment. In a prospective cohort study of $435 \mathrm{HBeAg}$-positive pregnant women, the $\mathrm{HBsAg}$ positivity rate of infants aged $8-12$ months was $8.3 \%$ in the breastfeeding group and $9.2 \%$ in the formula milk feeding group, which was not significantly different. ${ }^{558}$ Although studies on the safety of breastfeeding during the administration of NAs are very limited, recent studies in HIV-positive mother taking tenofovir DF have reported very low concentrations of the drug found in breast milk and that tenofovir is not absorbed through the intestines of infants. Therefore, considering the degree of liver disease in pregnant women and the risk of infants and young children, tenofovir DF can be carefully administered. ${ }^{599-561}$ Based on this, the World Health Organization recommends the use of tenofovir DF during pregnancy and lactation. ${ }^{562}$

Antiviral treatment for preventing MTCT: In the case of pregnant women with high serum HBV DNA levels $(\geq 200,000 \mathrm{IJ} / \mathrm{mL})$, postnatal HBIG injections and sequential immunization for the prevention of MTCT have a high failure rate. ${ }^{563,564}$ Therefore, there is a growing interest in lowering the MTCT rate through NAs during pregnancy.

- Lamivudine or telbivudine: In one prospective study of HBeAg-positive pregnant women with high serum HBV DNA levels $\left(>10^{7}\right.$ copies $\left./ \mathrm{mL}\right)$, they were treated with lamivudine from week 24 to week 32 in addition to neonatal passive-active immunoprophylaxis. The HBsAg-positivity rates of infants at 1 year after birth were significantly lower in the treatment group $(0 \%$, 0/94) compared to the placebo group $(7.7 \%, 7 / 91) .{ }^{565}$ Another prospective study included pregnant with high serum HBV DNA levels $\left(>10^{6}\right.$ copies $/ \mathrm{mL}$ ) who were treated with telbivudine starting at week 12-30 until birth in addition to neonatal passive-active immunoprophylaxis as the treatment group. The HBsAg-positivity rates of infants at 6 months after birth were also significantly lower in the treatment group $(0 \%, 0 / 54)$ compared to the placebo group $(8.6 \%, 3 / 35) .{ }^{566}$

- Tenofovir DF: In a non-randomized prospective study on administration of tenofovir DF starting at 30-32 weeks of gestation until 1 month postpartum, the HBsAg positivity rate of infants at 6 months of age was significantly lower in the experimental group $(1.5 \% \text { vs. } 10.7 \%)^{567}$ In another non-blinded, randomized, prospective study in which tenofovir DF was administered over the same period, the per-protocol analysis showed that the HBsAg positivity rate of infants at 24 week of birth was significantly lower in the experimental group ( $0 \%$ vs. $7 \%$ ). ${ }^{568}$ In a meta-analysis of ten studies using tenofovir $\mathrm{DF}$, including the above studies, tenofovir DF was reported to reduce MTCT by $77 \%{ }^{569}$ However, in a recent 
double-blind, randomized, prospective study, tenofovir DF was starting at 28 weeks of gestation until 2 months after birth, the difference in HBsAg positivity in infants at 6 months of age was insignificant (0\% vs. $2 \%) .{ }^{570}$ It should be noted that in that study, hepatitis $B$ vaccinations were performed 5 times after birth and the rate of MTCT was relatively low in the control group. In a recent meta-analysis of the two randomized prospective studies, there was no significant difference in the intention-to-treat analysis results, but the per-protocol analysis showed that tenofovir DF reduced MTCT by $98 \%{ }^{571}$ Therefore, the administration of NAs in pregnant women with serum HBV DNA levels of 200,000 IU/mL or more beginning at 24 to 32 weeks of gestation until 2 to 12 weeks postpartum can minimize the MTCT rate. However, in the case of pregnant women who do not meet into the general criteria for treatment for $\mathrm{CHB}$, the decision whether to administer NAs to prevent MTCT should be individualized and consider the timing of drug administration, the timing of withdrawal, and the preference of the pregnant women.

\section{[Recommendations]}

1. The administration of NAs in pregnant women or in patients preparing for pregnancy should follow the general principles of CHB treatment. Therapy should be carefully chosen considering the short- and long-term effects on both the mother and fetus, and tenofovir DF is currently recommended. (B1)

2. Child-bearing is contraindicated during peginterferon alfa treatment, and it should not be used in pregnant women. (A1)

3. If a CHB patient becomes pregnant while taking an NA other than tenofovir DF, it is recommended to switch the medication to tenofovir DF, which is relatively safe for the fetus as well as for pregnant women and is not contraindicated during breastfeeding. (B1)

4. There are no limitations regarding breastfeeding in $\mathrm{CHB}$ patients without antiviral treatment. (B1)

5. For pregnant women with serum HBV DNA levels $>200,000 \mathrm{IU} / \mathrm{mL}$, administration of tenofovir DF is recommended for the prevention of MTCT (A2), starting at 24-32 weeks of gestation and stopping 2-12 weeks after delivery. (B1)

\section{Children and adolescents}

Providing HBIG and HBV vaccines to newborns of HBsAg-positive mothers within 12 hours of birth can prevent $90-95 \%$ of cases of perinatal infection. ${ }^{76,77}$ Ninety percent of infants infected as neonates progress to chronic infection. Most children remain in the immune-tolerant phase until late childhood or adolescence; however, some children progress to the immune-active phase. The spontaneous $\mathrm{HBeAg}$ seroconversion rate in Korean children is $4.6 \%, 7.1 \%$, and $28.0 \%$ for patients aged $<6,6-12$, and $>12$ years, respectively. ${ }^{572}$ Children who are in the immune-active phase are usually asymptomatic. If ALT is continuously elevated in children with CHB, serum HBV DNA levels should be examined to confirm viral replication. In a study of 104 children and adolescents in Taiwan with a median follow-up of 23.7 years, spontaneous $\mathrm{HBeAg}$ seroconversion could be predicted in patients with ALT levels $>60 \mathrm{IU} / \mathrm{L}^{573}$

If long-term treatment is expected in children with $\mathrm{CHB}$, a prudent decision should be made based on the adverse effects of the antiviral treatment and the potential for antiviral resistance to affect future therapies. The treatment window should not be missed because cirrhosis can occur even in the patient's twenties, and HCC can occur later in life. The goals of CHB therapy are to suppress viral replication, reduce liver inflammation, reverse liver fibrosis, and prevent cirrhosis and HCC. Treating children in the immune-tolerant phase is not beneficial, and there is a high risk of developing antiviral resistance, which would limit treatment options later in life. However, a recent small-scale, randomized, prospective study reported that $\mathrm{HBeAg}$ seroconversion rates was $32.6 \%$ and $\mathrm{HBsAg}$ loss rates was $21.6 \%$ after 72 weeks of interferon-only or sequential lamivudine treatment. ${ }^{574}$

Children with a persistently elevated serum ALT level should be evaluated for active viral replication, including measuring HBV DNA levels. Children should be considered for treatment when their serum ALT levels are $\geq 2$ times the ULN for at least 6 months and their HBV DNA levels are $\geq 20,000 \mathrm{IU} / \mathrm{mL}$ (for HBeAg-positive children) or $\geq 2,000 \mathrm{IU} / \mathrm{mL}$ (for HBeAg-negative children). It is important to rule out other causes of ALT elevation, such as nonalcoholic fatty liver disease, before treatment and necessary to consider any family history of cirrhosis or HCC. Liver biopsy is helpful in the decision to treat, especially for children with moderate-tosevere necroinflammation or significant fibrosis $(\geq F 2) .{ }^{575}$

\section{Interferon alfa}

Interferon alfa is approved in children older than 12 months, and its advantages include a finite duration of treatment and no development of antiviral resistance. A randomized controlled trial of interferon alfa therapy involving children with $\mathrm{CHB}$ aged 1 to 17 years found that $36 \%$ of those with a baseline ALT level of at least 2 times the ULN lost HBeAg by the end of treatment. HBsAg seroconversion occurred in $10 \%$ of the treated children. ${ }^{576}$ Factors predictive of a positive response among children are age less than 5 years, low serum HBV DNA levels, and active inflammation as 
determined from a liver biopsy. ${ }^{577}$ The recommended treatment regimen for interferon alfa is 6 million $\mathrm{IU} / \mathrm{m}^{2}$ three times per week via subcutaneous injection for 24 weeks. The adverse effects include fever, flu-like symptoms, bone marrow suppression, depression, and transient growth delay. In a recent phase 3 study, pediatric and adolescent CHB patients between 3 and 16 years of age were treated with peginterferon alfa 2a for 48 weeks. The HBeAg seroconversion rate was significantly higher in the peginterferon alfa 2a group (25.7\%) than in the control group (6\%) at 24 weeks after the treatment completion, and the HBsAg disappearance rate, virological response rate, and ALT normalization rate were all significantly high. ${ }^{578}$

\section{Entecavir}

Entecavir and tenofovir DF are potent NAs with a high barrier to resistance, and entecavir is considered the first-line therapy in children older than 2 years. In a randomized controlled study involving 180 children aged 2 to 17 years with $\mathrm{HBeAg}$-positive CHB, the $\mathrm{HBeAg}$ seroconversion and HBV DNA $<50 \mathrm{IU} / \mathrm{mL}$ rates at week 48 were significantly higher with entecavir than placebo $(24.2 \%$ vs. $3.3 \%)$. The cumulative probability of entecavir resistance at 1 and 2 years was $0.6 \%$ and $2.6 \%$, respectively. Entecavir showed no difference in safety compared with placebo. ${ }^{579} \mathrm{~A}$ small-scale retrospective study of Koran pediatric and adolescent patients given entecavir reported second-year virological response (serum HBV DNA $<20 \mathrm{IU} / \mathrm{mL}$ ) and $\mathrm{HBeAg}$ seroconversion rates of $78.6 \%$ and $35.7 \%$, respectively. ${ }^{580}$ Entecavir is administered at a daily dose of $0.015 \mathrm{mg} / \mathrm{kg}$ (up to $0.5 \mathrm{mg}$ ).

\section{Tenofovir DF}

Tenofovir DF is approved for use in persons older than 12 years. A randomized controlled trial of tenofovir DF in adolescents aged 12 to 18 years reported that the rate of a virological response (HBV DNA <400 copies/mL) at week 72 was significantly higher in patients who received tenofovir $D F(n=52)$ than in those who received placebo $(n=54)(89 \%$ vs. $0 \%){ }^{581}$ No resistance to tenofovir developed during 72 weeks. The rate of grade $3 / 4$ adverse events was higher among patients treated with placebo (24\%) than those treated with tenofovir DF (10\%). In a small-scale study in Korean children and adolescents using tenofovir $D F$, the virological response (serum HBV DNA $<357 \mathrm{IU} / \mathrm{mL}$ ) was $93.8 \%$ at week 48 and $100 \%$ at week $96 .{ }^{582}$ The rate of HBeAg loss associated with undetectable HBV DNA was 41.7\% (5/12) at week 96. Tenofovir DF is administered at a daily dose of $8 \mathrm{mg} / \mathrm{kg}$ (up to $300 \mathrm{mg}$ ).

\section{Lamivudine}

Lamivudine is approved in children older than 2 years. A randomized controlled study of lamivudine involving children aged 2-17 years found that loss of HBeAg at 52 weeks of treatment occurred in $34 \%$ of those with a baseline ALT level of at least $\geq 2$ times the ULN and that the resistance rate was $18 \%{ }^{583}$ The antiviral resistance rate was $64 \%$ in children who received lamivudine for 3 years ${ }^{584}$ Other studies of Korean children found that the HBeAg seroconversion rates after 2 and 3 years of treatment were $65 \%$ and $70 \%$, respectively. Loss of HBsAg was observed in $20 \%$ of children after 2 years of lamivudine treatment, and the resistance rates at 1 and 2 years of treatment were $10 \%$ and $23 \%$, respectively. ${ }^{585,586}$ Lamivudine is orally administered at a dose of 3 $\mathrm{mg} / \mathrm{kg} /$ day with a maximum dose of $100 \mathrm{mg} /$ day. If lamivudine resistance develops, it should be treated in accordance with the guidelines for antiviral resistance management in adults. In a small-scale study of lamivudine-resistant pediatric patients in Korea, the virological response rate at week 24 was significantly higher $(P=0.029)$ in both lamivudine-adefovir combination therapy and in entecavir therapy alone compared to adefovir alone. ${ }^{587}$

The preferable duration of NA treatment in HBeAg-positive CHB children and adolescents is at least 1 year from initiation of treatment and more than 1 year after $\mathrm{HBeAg}$ seroconversion. In HBeAg-negative CHB children, the optimal treatment duration is not clear, but the standard recommendations for adults may be followed.

\section{[Recommendations]}

1. In HBeAg-positive CHB patients with HBV DNA levels $\geq 20,000$ $\mathrm{IU} / \mathrm{mL}$ or HBeAg-negative CHB patients with $\geq 2,000 \mathrm{IU} / \mathrm{mL}$, antiviral treatment is recommended if the ALT level is $\geq 2$ times the ULN or if liver biopsy shows moderate to severe necroinflammation or significant fibrosis $(\geq F 2)$. (A1)

2. Treatment with entecavir, tenofovir $D F$, or peginterferon alfa $2 \mathrm{a}$ is recommended in children and adolescent CHB patients. (A1)

3. If antiviral resistance develops during treatment, the recommendations for management of antiviral resistant $\mathrm{CHB}$ in adults given in the present guidelines should be followed. (B1)

\section{Authors' contribution}

Yim HJ, directed the guideline committee, and outlined and supervised the manuscript writing and editing; Yoon EL edited the manuscript. All the committee members participated drafting the manuscript; Yim HJ (preamble, and supplementary material), Yoon 
EL (epidemiology, natural history, prevention, and diagnosis and initial evaluation), Kim JH (treatment goal and aims, monitoring during antiviral treatment, and cessation of treatment and monitoring after antiviral treatment), Sinn $\mathrm{DH}$ (treatment indication, monitoring of patients who are not indicated for treatment, treatment strategy, and definition and predictors of antiviral treatment response); Lee HW (therapeutic agents and management in patients with renal dysfunction or metabolic bone disease); Park JY (antiviral resistance and management of antiviral resistance), Lee $\mathrm{JH}$ (management according to treatment response and management in patients with hepatocellular carcinoma), Kwon JH (therapeutic agents and management in patients with renal dysfunction or metabolic bone disease), Park $\mathrm{H}$ (management in patients with acute hepatitis $B$, patients on immunosuppression or chemotherapy, and liver transplant patients), and Lee SH (management in non-liver organ transplant recipients, coinfection with other viruses, pregnant women and women preparing for pregnancy, and children and adolescents) contributed to the manuscript writing.

\section{Conflicts of Interest}

Yim HJ: Received honoraria from Gilead, Yuhan, and Chongkundang Pharm. Received grants from Dong-A ST, Chongkundang Pharm, and Gilead.

Yoon EL: Received honoraria, consulted for, and received grant from Samil.

Kim JH: Received honoraria from Gilead, Abbvie, Chong kundang, Yuhan, Dong-A ST. Consulted for Gilead, Abbvie, Chongkundang, Dong-A ST. Received grants from Gilead, BMS, Abbvie, Chongkundang, Yuhan, Dong-A ST, Daewoong, Samjin, and KOWA.

Sinn DH: Received honoraria from Gilead, and Dong-A ST. Received grant from Dong-A ST.

Lee HW: Received honoraria from Gilead, Abbvie, Chongkundang, Yuhan, Dong-A ST, and Ildong.

Park JY: Received honoraria from Gilead, Yuhan, Abbvie, DongA ST, Hanmi, and Chongkundang. Received grants from Samil, Abbvie, Gilead, and Norvatis.

Lee JH: Nothing to declare.

Kwon JH: Received honoraria for lectures and consultation from Gilead, Abbvie, MSD, and BMS.

Park H: Received honoraria from Gilead. Received grants from Daewoong.

Lee SH: Received honoraria from Abbvie, BMS, and Yuhan.

\section{SUPPLEMENTARY MATERIAL}

Supplementary material is available at Clinical and Molecular Hepatology website (http://www.e-cmh.org)

\section{REFERENCES}

1. Schweitzer A, Horn J, Mikolajczyk RT, Krause G, Ott JJ. Estimations of worldwide prevalence of chronic hepatitis $B$ virus infection: a systematic review of data published between 1965 and 2013. Lancet 2015:386:1546-1555

2. Chae HB, Kim JH, Kim JK, Yim HJ. Current status of liver diseases in Korea: hepatitis B. The Korean Journal of Hepatology 2009;15 Suppl 6:S13-S24.

3. Kim BH, Park JW. Epidemiology of liver cancer in South Korea. Clin Mol Hepatol 2018;24:1-9.

4. Terrault NA, Bzowej NH, Chang KM, Hwang JP, Jonas MM, Murad $\mathrm{MH}$, et al. AASLD guidelines for treatment of chronic hepatitis $\mathrm{B}$. Hepatology 2016;63:261-283.

5. Hui CK, Leung N, Yuen ST, Zhang HY, Leung KW, Lu L, et al. Natural history and disease progression in Chinese chronic hepatitis $B$ patients in immune-tolerant phase. Hepatology 2007;46:395-401.

6. Tran TT. Immune tolerant hepatitis B: a clinical dilemma. Gastroenterol Hepatol (NY) 2011;7:511-516.

7. Chu CM, Hung SJ, Lin J, Tai DI, Liaw YF. Natural history of hepatitis $B$ e antigen to antibody seroconversion in patients with normal serum aminotransferase levels. Am J Med 2004;116:829-834.

8. Mason WS, Gill US, Litwin S, Zhou Y, Peri S, Pop 0, et al. HBV DNA integration and clonal hepatocyte expansion in chronic hepatitis B patients considered immune tolerant. Gastroenterology 2016;151:986-998.e4.

9. Livingston SE, Simonetti JP, Bulkow LR, Homan CE, Snowball MM, Cagle $\mathrm{HH}$, et al. Clearance of hepatitis $\mathrm{B}$ e antigen in patients with chronic hepatitis B and genotypes A, B, C, D, and F. Gastroenterology 2007;133:1452-1457.

10. Tsai SL, Chen PJ, Lai MY, Yang PM, Sung JL, Huang JH, et al. Acute exacerbations of chronic type $B$ hepatitis are accompanied by increased $T$ cell responses to hepatitis $B$ core and e antigens. Implications for hepatitis B e antigen seroconversion. J Clin Invest 1992;89:87-96.

11. Lee PI, Chang MH, Lee CY, Hsu HY, Chen JS, Chen PJ, et al. Changes of serum hepatitis B virus DNA and aminotransferase levels during the course of chronic hepatitis B virus infection in children. Hepatology 1990;12(4 Pt 1):657-660.

12. Lok AS, Lai CL. Acute exacerbations in Chinese patients with chronic hepatitis B virus (HBV) infection. Incidence, predisposing factors and etiology. J Hepatol 1990;10:29-34. 
13. McMahon BJ. The natural history of chronic hepatitis B virus infection. Hepatology 2009;49(5 Suppl):S45-S55.

14. Bortolotti F, Guido M, Bartolacci S, Cadrobbi P, Crivellaro C, Noventa $F$, et al. Chronic hepatitis $B$ in children after e antigen seroclearance: final report of a 29-year longitudinal study. Hepatology 2006;43:556-562.

15. Hsu YS, Chien RN, Yeh CT, Sheen IS, Chiou HY, Chu CM, et al. Long-term outcome after spontaneous $\mathrm{HBeAg}$ seroconversion in patients with chronic hepatitis B. Hepatology 2002;35:1522-1527.

16. Lok AS, Lai CL, Wu PC, Leung EK, Lam TS. Spontaneous hepatitis $B$ e antigen to antibody seroconversion and reversion in Chinese patients with chronic hepatitis B virus infection. Gastroenterology 1987:92:1839-1843.

17. Yim HJ, Lok AS. Natural history of chronic hepatitis B virus infection: what we knew in 1981 and what we know in 2005. Hepatology 2006;43(2 Suppl 1):S173-S181.

18. Sheen IS, Liaw YF, Tai DI, Chu CM. Hepatic decompensation associated with hepatitis B e antigen clearance in chronic type B hepatitis. Gastroenterology 1985;89:732-735.

19. Martinot-Peignoux M, Boyer N, Colombat M, Akremi R, Pham BN, Ollivier $S$, et al. Serum hepatitis B virus DNA levels and liver histology in inactive HBsAg carriers. J Hepatol 2002;36:543-546.

20. Zacharakis GH, Koskinas J, Kotsiou S, Papoutselis M, Tzara F, Vafeiadis $N$, et al. Natural history of chronic HBV infection: a cohort study with up to 12 years follow-up in North Greece (part of the Interreg I-II/EC-project). J Med Virol 2005;77:173-179.

21. de Franchis R, Meucci G, Vecchi M, Tatarella M, Colombo M, Del Ninno $E$, et al. The natural history of asymptomatic hepatitis $B$ surface antigen carriers. Ann Intern Med 1993;118:191-194.

22. Fattovich $G$. Natural history and prognosis of hepatitis B. Semin Liver Dis 2003;23:47-58.

23. Chen YC, Sheen IS, Chu CM, Liaw YF. Prognosis following spontaneous HBsAg seroclearance in chronic hepatitis $B$ patients with or without concurrent infection. Gastroenterology 2002;123:10841089.

24. Lok AS, McMahon BJ. Chronic hepatitis B: update 2009. Hepatology 2009;50:661-662.

25. Funk ML, Rosenberg DM, Lok AS. World-wide epidemiology of HBeAg-negative chronic hepatitis B and associated precore and core promoter variants. J Viral Hepat 2002;9:52-61.

26. Lok AS, Akarca U, Greene $S$. Mutations in the pre-core region of hepatitis $B$ virus serve to enhance the stability of the secondary structure of the pre-genome encapsidation signal. Proc Natl Acad Sci U S A 1994;91:4077-4081.

27. Okamoto H, Tsuda F, Akahane Y, Sugai Y, Yoshiba M, Moriyama K, et al. Hepatitis B virus with mutations in the core promoter for an e antigen-negative phenotype in carriers with antibody to e antigen. J Virol 1994;68:8102-8110.
28. Croagh CM, Bell SJ, Slavin J, Kong YX, Chen RY, Locarnini S, et al. Increasing hepatitis $B$ viral load is associated with risk of significant liver fibrosis in $\mathrm{HBeAg}$-negative but not $\mathrm{HBeAg}$-positive chronic hepatitis B. Liver Int 2010;30:1115-1122.

29. Yoo BC, Park JW, Kim HJ, Lee DH, Cha YJ, Park SM. Precore and core promoter mutations of hepatitis B virus and hepatitis B e antigen-negative chronic hepatitis B in Korea. J Hepatol 2003;38:98103.

30. Raimondo G, Allain JP, Brunetto MR, Buendia MA, Chen DS, Colombo $M$, et al. Statements from the Taormina expert meeting on occult hepatitis B virus infection. J Hepatol 2008;49:652-657.

31. Chu CM, Liaw YF. HBsAg seroclearance in asymptomatic carriers of high endemic areas: appreciably high rates during a long-term follow-up. Hepatology 2007;45:1187-1192.

32. Liu J, Yang $\mathrm{HI}$, Lee $\mathrm{MH}$, Lu SN, Jen $\mathrm{CL}$, Wang LY, et al. Incidence and determinants of spontaneous hepatitis B surface antigen seroclearance: a community-based follow-up study. Gastroenterology 2010;139:474-482.

33. Liaw YF, Sheen IS, Chen TJ, Chu CM, Pao CC. Incidence, determinants and significance of delayed clearance of serum HBsAg in chronic hepatitis B virus infection: a prospective study. Hepatology 1991;13:627-631.

34. Nam SW, Jung JJ, Bae SH, Choi JY, Yoon SK, Cho SH, et al. Clinical outcomes of delayed clearance of serum HBsAG in patients with chronic HBV infection. Korean J Intern Med 2007;22:73-76.

35. Kuang XJ, Jia RR, Huo RR, Yu JJ, Wang JJ, Xiang BD, et al. Systematic review of risk factors of hepatocellular carcinoma after hepatitis B surface antigen seroclearance. J Viral Hepat 2018;25:10261037.

36. Yip TC, Chan HL, Wong VW, Tse YK, Lam KL, Wong GL. Impact of age and gender on risk of hepatocellular carcinoma after hepatitis B surface antigen seroclearance. J Hepatol 2017;67:902-908.

37. Lee KJ, Han KH, Chun JY, Moon YM, Lee SI, Park IS, et al. Natural history of chronic hepatitis type B throughout long-term follow-up. Korean J Gastroenterol 1997;29:343-351.

38. Liu S, Zhang H, Gu C, Yin J, He Y, Xie J, et al. Associations between hepatitis $B$ virus mutations and the risk of hepatocellular carcinoma: a meta-analysis. J Natl Cancer Inst 2009;101:1066-1082.

39. Yang HI, Yeh SH, Chen PJ, lloeje UH, Jen CL, Su J, et al. Associations between hepatitis $\mathrm{B}$ virus genotype and mutants and the risk of hepatocellular carcinoma. J Natl Cancer Inst 2008;100:11341143.

40. Tseng TC, Liu CJ, Yang HC, Chen CL, Yang WT, Tsai CS, et al. Higher proportion of viral basal core promoter mutant increases the risk of liver cirrhosis in hepatitis B carriers. Gut 2015;64:292-302.

41. Jang JW, Chun JY, Park YM, Shin SK, Yoo W, Kim SO, et al. Mutational complex genotype of the hepatitis $B$ virus $X$ /precore regions as a novel predictive marker for hepatocellular carcinoma. Cancer 
Sci 2012;103:296-304.

42. Kim JK, Chang HY, Lee JM, Baatarkhuu O, Yoon YJ, Park JY, et al. Specific mutations in the enhancer II/core promoter/precore regions of hepatitis $B$ virus subgenotype $C 2$ in Korean patients with hepatocellular carcinoma. J Med Virol 2009;81:1002-1008.

43. Kao JH, Chen PJ, Lai MY, Chen DS. Basal core promoter mutations of hepatitis $B$ virus increase the risk of hepatocellular carcinoma in hepatitis B carriers. Gastroenterology 2003;124:327-334.

44. Wei F, Zheng Q, Li M, Wu M. The association between hepatitis B mutants and hepatocellular carcinoma: a meta-analysis. Medicine (Baltimore) 2017;96:e6835.

45. Chen CJ, Yang HI, Su J, Jen CL, You SL, Lu SN, et al. Risk of hepatocellular carcinoma across a biological gradient of serum hepatitis B virus DNA level. JAMA 2006:295:65-73.

46. Fattovich G, Stroffolini T, Zagni I, Donato F. Hepatocellular carcinoma in cirrhosis: incidence and risk factors. Gastroenterology 2004;127(5 Suppl 1):S35-S50.

47. Iloeje UH, Yang HI, Su J, Jen CL, You SL, Chen CJ, et al. Predicting cirrhosis risk based on the level of circulating hepatitis B viral load. Gastroenterology 2006;130:678-686.

48. Bravi F, Tavani A, Bosetti C, Boffetta P, La Vecchia C. Coffee and the risk of hepatocellular carcinoma and chronic liver disease: a systematic review and meta-analysis of prospective studies. Eur J Cancer Prev 2017;26:368-377.

49. Kennedy OJ, Roderick P, Buchanan R, Fallowfield JA, Hayes PC, Parkes J. Coffee, including caffeinated and decaffeinated coffee, and the risk of hepatocellular carcinoma: a systematic review and dose-response meta-analysis. BMJ Open 2017;7:e013739.

50. Petrick JL, Freedman ND, Graubard BI, Sahasrabuddhe VV, Lai GY, Alavanja MC, et al. Coffee consumption and risk of hepatocellular carcinoma and intrahepatic cholangiocarcinoma by sex: the liver cancer pooling project. Cancer Epidemiol Biomarkers Prev 2015:24:1398-1406.

51. Zhou YY, Zhu GQ, Liu T, Zheng JN, Cheng Z, Zou TT, et al. Systematic review with network meta-analysis: antidiabetic medication and risk of hepatocellular carcinoma. Sci Rep 2016;6:33743.

52. Hwang IC, Chang J, Kim K, Park SM. Aspirin use and risk of hepatocellular carcinoma in a national cohort study of Korean adults. Sci Rep 2018;8:4968.

53. Lee M, Chung GE, Lee JH, Oh S, Nam JY, Chang Y, et al. Antiplatelet therapy and the risk of hepatocellular carcinoma in chronic hepatitis B patients on antiviral treatment. Hepatology 2017;66:15561569.

54. Chen $\mathrm{Cl}$, Kuan CF, Fang YA, Liu SH, Liu JC, Wu LL, et al. Cancer risk in HBV patients with statin and metformin use: a population-based cohort study. Medicine (Baltimore) 2015;94:e462.

55. Hsiang JC, Wong GL, Tse YK, Wong VW, Yip TC, Chan HL. Statin and the risk of hepatocellular carcinoma and death in a hospital- based hepatitis B-infected population: a propensity score landmark analysis. J Hepatol 2015;63:1190-1197.

56. Kim G, Jang SY, Nam CM, Kang ES. Statin use and the risk of hepatocellular carcinoma in patients at high risk: a nationwide nested case-control study. J Hepatol 2018;68:476-484.

57. Pradelli D, Soranna D, Scotti L, Zambon A, Catapano A, Mancia G, et al. Statins and primary liver cancer: a meta-analysis of observational studies. Eur J Cancer Prev 2013;22:229-234.

58. Singh S, Singh PP, Singh AG, Murad MH, Sanchez W. Statins are associated with a reduced risk of hepatocellular cancer: a systematic review and meta-analysis. Gastroenterology 2013;144:323-332.

59. Zhou YY, Zhu GQ, Wang Y, Zheng JN, Ruan LY, Cheng Z, et al. Systematic review with network meta-analysis: statins and risk of hepatocellular carcinoma. Oncotarget 2016;7:21753-21762.

60. Yang HI, Yuen MF, Chan HL, Han KH, Chen PJ, Kim DY, et al. Risk estimation for hepatocellular carcinoma in chronic hepatitis $B$ (REACH-B): development and validation of a predictive score. Lancet Oncol 2011;12:568-574.

61. Lee HW, Yoo EJ, Kim BK, Kim SU, Park JY, Kim DY, et al. Prediction of development of liver-related events by transient elastography in hepatitis B patients with complete virological response on antiviral therapy. Am J Gastroenterol 2014;109:1241-1249.

62. Jung KS, Kim SU, Song K, Park JY, Kim DY, Ahn SH, et al. Validation of hepatitis $B$ virus-related hepatocellular carcinoma prediction models in the era of antiviral therapy. Hepatology 2015;62:17571766.

63. Papatheodoridis G, Dalekos G, Sypsa V, Yurdaydin C, Buti M, Goulis J, et al. PAGE-B predicts the risk of developing hepatocellular carcinoma in Caucasians with chronic hepatitis B on 5-year antiviral therapy. J Hepatol 2016;64:800-806.

64. Kim MN, Hwang SG, Rim KS, Kim BK, Park JY, Kim DY, et al. Validation of PAGE-B model in Asian chronic hepatitis B patients receiving entecavir or tenofovir. Liver Int 2017;37:1788-1795.

65. Kim JH, Kim YD, Lee M, Jun BG, Kim TS, Suk KT, et al. Modified PAGE-B score predicts the risk of hepatocellular carcinoma in Asians with chronic hepatitis B on antiviral therapy. J Hepatol 2018;69:1066-1073.

66. Cho EJ, Kim SE, Suk KT, An J, Jeong SW, Chung WJ, et al. Current status and strategies for hepatitis B control in Korea. Clin Mol Hepatol 2017;23:205-211.

67. Terrault NA, Lok ASF, McMahon BJ, Chang KM, Hwang JP, Jonas $M M$, et al. Update on prevention, diagnosis, and treatment of chronic hepatitis B: AASLD 2018 hepatitis B guidance. Hepatology 2018:67:1560-1599.

68. Kim DK, Riley LE, Harriman KH, Hunter P, Bridges CB. Advisory Committee on Immunization Practices recommended immunization schedule for adults aged 19 years or older - United States, 2017. MMWR Morb Mortal Wkly Rep 2017;66:136-138. 
69. Mast EE, Margolis HS, Fiore AE, Brink EW, Goldstein ST, Wang SA, et al. A comprehensive immunization strategy to eliminate transmission of hepatitis B virus infection in the United States: recommendations of the Advisory Committee on Immunization Practices (ACIP) part 1: immunization of infants, children, and adolescents. MMWR Recomm Rep 2005;54(RR-16):1-31.

70. Mast EE, Weinbaum CM, Fiore AE, Alter MJ, Bell BP, Finelli L, et al. A comprehensive immunization strategy to eliminate transmission of hepatitis B virus infection in the United States: recommendations of the Advisory Committee on Immunization Practices (ACIP) Part II: immunization of adults. MMWR Recomm Rep 2006;55(RR-16):133; quiz CE1-4.

71. U.S. Public Health Service. Updated U.S. Public Health Service Guidelines for the management of occupational exposures to HBV, HCV, and HIV and recommendations for postexposure prophylaxis. MMWR Recomm Rep 2001;50(RR-11):1-52.

72. Lok AS, Lai CL, Wu PC. Prevalence of isolated antibody to hepatitis $B$ core antigen in an area endemic for hepatitis $B$ virus infection: implications in hepatitis B vaccination programs. Hepatology 1988;8:766-770.

73. McMahon BJ, Parkinson AJ, Helminiak C, Wainwright RB, Bulkow $L$, Kellerman-Douglas $A$, et al. Response to hepatitis $B$ vaccine of persons positive for antibody to hepatitis B core antigen. Gastroenterology 1992;103:590-594.

74. Keeffe EB. Hepatitis A and B superimposed on chronic liver disease: vaccine-preventable diseases. Trans Am Clin Climatol Assoc 2006;117:227-237; discussion 237-238.

75. Craig AS, Schaffner W. Prevention of hepatitis A with the hepatitis A vaccine. N Engl J Med 2004;350:476-481.

76. Beasley RP, Hwang LY, Lee GC, Lan CC, Roan CH, Huang FY, et al. Prevention of perinatally transmitted hepatitis $B$ virus infections with hepatitis $B$ immune globulin and hepatitis B vaccine. Lancet 1983;2:1099-1102.

77. Eke AC, Eleje GU, Eke UA, Xia Y, Liu J. Hepatitis B immunoglobulin during pregnancy for prevention of mother-to-child transmission of hepatitis B virus. Cochrane Database Syst Rev 2017;2:CD008545.

78. Chen X, Chen J, Wen J, Xu C, Zhang S, Zhou YH, et al. Breastfeeding is not a risk factor for mother-to-child transmission of hepatitis B virus. PLoS One 2013;8:e55303.

79. Beasley RP, Stevens CE, Shiao IS, Meng HC. Evidence against breast-feeding as a mechanism for vertical transmission of hepatitis B. Lancet 1975;2:740-741.

80. Chevillotte G, Durbec JP, Gerolami A, Berthezene P, Bidart JM, Camatte R. Interaction between hepatitis $b$ virus and alcohol consumption in liver cirrhosis. An epidemiologic study. Gastroenterology 1983;85:141-145.

81. Villa E, Rubbiani L, Barchi T, Ferretti I, Grisendi A, De Palma M, et al. Susceptibility of chronic symptomless HBsAg carriers to ethanol- induced hepatic damage. Lancet 1982;2:1243-1244.

82. Trichopoulos D, Bamia C, Lagiou P, Fedirko V, Trepo E, Jenab M, et al. Hepatocellular carcinoma risk factors and disease burden in a European cohort: a nested case-control study. J Natl Cancer Inst 2011;103:1686-1695.

83. Jee SH, Ohrr H, Sull JW, Samet JM. Cigarette smoking, alcohol drinking, hepatitis $B$, and risk for hepatocellular carcinoma in Korea. J Natl Cancer Inst 2004;96:1851-1856.

84. Yu MW, Lin CL, Liu CJ, Yang SH, Tseng YL, Wu CF. Influence of metabolic risk factors on risk of hepatocellular carcinoma and liverrelated death in men with chronic hepatitis B: a large cohort study. Gastroenterology 2017;153:1006-1017.e5.

85. Wong GL, Chan HL, Yu Z, Chan AW, Choi PC, Chim AM, et al. Coincidental metabolic syndrome increases the risk of liver fibrosis progression in patients with chronic hepatitis B--a prospective cohort study with paired transient elastography examinations. Aliment Pharmacol Ther 2014;39:883-893.

86. Chan AW, Wong GL, Chan HY, Tong JH, Yu YH, Choi PC, et al. Concurrent fatty liver increases risk of hepatocellular carcinoma among patients with chronic hepatitis B. J Gastroenterol Hepatol 2017:32:667-676

87. Kim JH, Sinn DH, Gwak GY, Kang W, Paik YH, Choi MS, et al. Insulin resistance and the risk of hepatocellular carcinoma in chronic hepatitis B patients. J Gastroenterol Hepatol 2017;32:1100-1106.

88. Kim NH, Cho YK, Kim BI, Kim HJ. Effect of metabolic syndrome on the clinical outcomes of chronic hepatitis B patients with nucleos(t) ide analogues treatment. Dig Dis Sci 2018;63:2792-2799.

89. Lai CL, Lau JY, Yeoh EK, Chang WK, Lin HJ. Significance of isolated anti-HBC seropositivity by ELISA: implications and the role of radioimmunoassay. J Med Virol 1992;36:180-183.

90. Sánchez-Quijano A, Jauregui JI, Leal M, Pineda JA, Castilla A, Abad MA, et al. Hepatitis $B$ virus occult infection in subjects with persistent isolated anti-HBc reactivity. J Hepatol 1993;17:288-293.

91. Raimondo G, Navarra G, Mondello S, Costantino L, Colloredo G, Cucinotta $E$, et al. Occult hepatitis B virus in liver tissue of individuals without hepatic disease. J Hepatol 2008;48:743-746.

92. Said ZN. An overview of occult hepatitis B virus infection. World J Gastroenterol 2011;17:1927-1938.

93. Moon HW, Cho JH, Hur M, Yun YM, Choe WH, Kwon SY, et al. Laboratory characteristics of recent hepatitis $A$ in Korea: ongoing epidemiological shift. World J Gastroenterol 2010;16:1115-1118.

94. Jacobsen KH, Koopman JS. Declining hepatitis A seroprevalence: a global review and analysis. Epidemiol Infect 2004;132:1005-1022.

95. Song HJ, Kim TH, Song JH, Oh HJ, Ryu KH, Yeom HJ, et al. Emerging need for vaccination against hepatitis $A$ virus in patients with chronic liver disease in Korea. J Korean Med Sci 2007;22:218-222.

96. Yoon EL, Sinn DH, Lee HW, Kim JH. Current status and strategies for the control of viral hepatitis A in Korea. Clin Mol Hepatol 
2017;23:196-204.

97. European Association for the Study of the Liver. EASL 2017 Clinical Practice Guidelines on the management of hepatitis B virus infection. J Hepatol 2017;67:370-398.

98. World Health Organization (WHO). Guidelines for the Prevention, Care and Treatment of Persons with Chronic Hepatitis B Infection. Geneva: WHO, 2015:38.

99. Perrillo RP, Lai $C L$, Liaw YF, Dienstag JL, Schiff ER, Schalm SW, et al. Predictors of HBeAg loss after lamivudine treatment for chronic hepatitis B. Hepatology 2002;36:186-194.

100. Prati D, Taioli E, Zanella A, Della Torre E, Butelli S, Del Vecchio E, et al. Updated definitions of healthy ranges for serum alanine aminotransferase levels. Ann Intern Med 2002;137:1-10.

101. Shim JJ, Kim JW, Oh CH, Lee YR, Lee JS, Park SY, et al. Serum alanine aminotransferase level and liver-related mortality in patients with chronic hepatitis B: a large national cohort study. Liver Int 2018;38:1751-1759.

102. Kariv R, Leshno M, Beth-Or A, Strul H, Blendis L, Kokia E, et al. Reevaluation of serum alanine aminotransferase upper normal limit and its modulating factors in a large-scale population study. Liver Int 2006;26:445-450.

103. Pawlotsky JM. Hepatitis B virus (HBV) DNA assays (methods and practical use) and viral kinetics. J Hepatol 2003;39 Suppl 1:S31-S35.

104. Chu CM, Liaw YF. Genotype C hepatitis B virus infection is associated with a higher risk of reactivation of hepatitis $B$ and progression to cirrhosis than genotype $\mathrm{B}$ : a longitudinal study of hepatitis $B$ e antigen-positive patients with normal aminotransferase levels at baseline. J Hepatol 2005;43:411-417.

105. Sumi H, Yokosuka O, Seki N, Arai M, Imazeki F, Kurihara T, et al. Influence of hepatitis $B$ virus genotypes on the progression of chronic type B liver disease. Hepatology 2003;37:19-26.

106. Yu MW, Yeh SH, Chen PJ, Liaw YF, Lin CL, Liu CJ, et al. Hepatitis $B$ virus genotype and DNA level and hepatocellular carcinoma: a prospective study in men. J Natl Cancer Inst 2005;97:265-272.

107. Lin CL, Kao JH. Natural history of acute and chronic hepatitis B: the role of HBV genotypes and mutants. Best Pract Res Clin Gastroenterol 2017;31:249-255.

108. Kao JH, Wu NH, Chen PJ, Lai MY, Chen DS. Hepatitis B genotypes and the response to interferon therapy. J Hepatol 2000;33:9981002.

109. Keeffe EB, Dieterich DT, Han SH, Jacobson IM, Martin P, Schiff $E R$, et al. A treatment algorithm for the management of chronic hepatitis B virus infection in the United States: 2008 update. Clin Gastroenterol Hepatol 2008;6:1315-1341; quiz 1286.

110. Cornberg M, Wong VW, Locarnini S, Brunetto M, Janssen HLA, Chan $\mathrm{HL}$. The role of quantitative hepatitis B surface antigen revisited. J Hepatol 2017;66:398-411.

111. Nguyen T, Thompson AJ, Bowden S, Croagh C, Bell S, Desmond PV, et al. Hepatitis B surface antigen levels during the natural history of chronic hepatitis B: a perspective on Asia. J Hepatol 2010;52:508513.

112. Suh SJ, Bae SI, Kim JH, Kang K, Yeon JE, Byun KS. Clinical implications of the titer of serum hepatitis $B$ surface antigen during the natural history of hepatitis B virus infection. J Med Virol 2014;86:117-123.

113. Liu J, Yang HI, Lee $M H$, Jen $C L$, Batrla-Utermann $R$, Lu SN, et al. Serum levels of hepatitis $B$ surface antigen and DNA can predict inactive carriers with low risk of disease progression. Hepatology 2016;64:381-389.

114. Park H, Lee JM, Seo JH, Kim HS, Ahn SH, Kim DY, et al. Predictive value of HBsAg quantification for determining the clinical course of genotype C HBeAg-negative carriers. Liver Int 2012;32:796-802.

115. Tseng TC, Liu CJ, Yang HC, Su TH, Wang CC, Chen CL, et al. High levels of hepatitis $B$ surface antigen increase risk of hepatocellular carcinoma in patients with low HBV load. Gastroenterology 2012;142:1140-1149.e3; quiz e13-e14.

116. Sonneveld MJ, Hansen BE, Piratvisuth T, Jia JD, Zeuzem S, Gane $E$, et al. Response-guided peginterferon therapy in hepatitis $B$ antigen-positive chronic hepatitis B using serum hepatitis B surface antigen levels. Hepatology 2013;58:872-880.

117. Chang ML, Liaw YF, Hadziyannis SJ. Systematic review: cessation of long-term nucleos(t)ide analogue therapy in patients with hepatitis B e antigen-negative chronic hepatitis B. Aliment Pharmacol Ther 2015:42:243-257.

118. Lee HA, Seo YS, Park SW, Park SJ, Kim TH, Suh SJ, et al. Hepatitis $B$ surface antigen titer is a good indicator of durable viral response after entecavir off-treatment for chronic hepatitis B. Clin Mol Hepatol 2016;22:382-389.

119. Lai M, Hyatt BJ, Nasser I, Curry M, Afdhal NH. The clinical significance of persistently normal ALT in chronic hepatitis B infection. J Hepatol 2007:47:760-767.

120. Lee S, Kim DY. Non-invasive diagnosis of hepatitis B virus-related cirrhosis. World J Gastroenterol 2014;20:445-459.

121. Parikh P, Ryan JD, Tsochatzis EA. Fibrosis assessment in patients with chronic hepatitis B virus (HBV) infection. Ann Transl Med 2017; 5:40.

122. Wai CT, Greenson JK, Fontana RJ, Kalbfleisch JD, Marrero JA, Conjeevaram HS, et al. A simple noninvasive index can predict both significant fibrosis and cirrhosis in patients with chronic hepatitis $C$. Hepatology 2003;38:518-526

123. Jin W, Lin Z, Xin Y, Jiang $X$, Dong $Q$, Xuan S. Diagnostic accuracy of the aspartate aminotransferase-to-platelet ratio index for the prediction of hepatitis B-related fibrosis: a leading meta-analysis. BMC Gastroenterol 2012;12:14.

124. Xiao G, Yang J, Yan L. Comparison of diagnostic accuracy of aspartate aminotransferase to platelet ratio index and fibrosis-4 index 
for detecting liver fibrosis in adult patients with chronic hepatitis $B$ virus infection: a systemic review and meta-analysis. Hepatology 2015;61:292-302.

125. Vallet-Pichard A, Mallet V, Nalpas B, Verkarre V, Nalpas A, Dhalluin-Venier $V$, et al. FIB-4: an inexpensive and accurate marker of fibrosis in HCV infection. Comparison with liver biopsy and fibrotest. Hepatology 2007;46:32-36.

126. Kim BK, Kim DY, Park JY, Ahn SH, Chon CY, Kim JK, et al. Validation of FIB-4 and comparison with other simple noninvasive indices for predicting liver fibrosis and cirrhosis in hepatitis B virus-infected patients. Liver Int 2010;30:546-553.

127. Li Y, Chen Y, Zhao Y. The diagnostic value of the FIB-4 index for staging hepatitis B-related fibrosis: a meta-analysis. PLoS One 2014;9:e105728.

128. Wong GL. Update of liver fibrosis and steatosis with transient elastography (Fibroscan). Gastroenterol Rep (Oxf) 2013;1:19-26.

129. Lucidarme D, Foucher J, Le Bail B, Vergniol J, Castera L, Duburque $C$, et al. Factors of accuracy of transient elastography (fibroscan) for the diagnosis of liver fibrosis in chronic hepatitis C. Hepatology 2009;49:1083-1089.

130. Myers RP, Crotty P, Pomier-Layrargues G, Ma M, Urbanski SJ, Elkashab M. Prevalence, risk factors and causes of discordance in fibrosis staging by transient elastography and liver biopsy. Liver Int 2010;30:1471-1480.

131. Castera L, Forns X, Alberti A. Non-invasive evaluation of liver fibrosis using transient elastography. J Hepatol 2008;48:835-847.

132. Sandrin L, Fourquet B, Hasquenoph JM, Yon S, Fournier C, Mal F, et al. Transient elastography: a new noninvasive method for assessment of hepatic fibrosis. Ultrasound Med Biol 2003;29:1705-1713.

133. Castera L, Pinzani M. Biopsy and non-invasive methods for the diagnosis of liver fibrosis: does it take two to tango? Gut 2010;59:861-866.

134. Castera L. Noninvasive methods to assess liver disease in patients with hepatitis B or C. Gastroenterology 2012;142:1293-1302.e4.

135. Chon YE, Choi EH, Song KJ, Park JY, Kim DY, Han KH, et al. Performance of transient elastography for the staging of liver fibrosis in patients with chronic hepatitis B: a meta-analysis. PLoS One 2012;7:e44930.

136. Zhu X, Wang LC, Chen EQ, Chen XB, Chen LY, Liu L, et al. Prospective evaluation of FibroScan for the diagnosis of hepatic fibrosis compared with liver biopsy/AST platelet ratio index and FIB-4 in patients with chronic HBV infection. Dig Dis Sci 2011;56:27422749.

137. Chang W, Lee JM, Yoon JH, Han JK, Choi BI, Yoon JH, et al. Liver fibrosis staging with MR elastography: comparison of diagnostic performance between patients with chronic hepatitis $B$ and those with other etiologic causes. Radiology 2016;280:88-97.

138. Morisaka H, Motosugi U, Ichikawa S, Nakazawa T, Kondo T, Fu- nayama $\mathrm{S}$, et al. Magnetic resonance elastography is as accurate as liver biopsy for liver fibrosis staging. J Magn Reson Imaging 2018;47:1268-1275.

139. Bohte AE, de Niet A, Jansen L, Bipat S, Nederveen AJ, Verheij J, et al. Non-invasive evaluation of liver fibrosis: a comparison of ultrasound-based transient elastography and MR elastography in patients with viral hepatitis B and C. Eur Radiol 2014;24:638-648.

140. Ichikawa S, Motosugi U, Morisaka H, Sano K, Ichikawa T, Tatsumi $\mathrm{A}$, et al. Comparison of the diagnostic accuracies of magnetic resonance elastography and transient elastography for hepatic fibrosis. Magn Reson Imaging 2015;33:26-30.

141. Toguchi M, Tsurusaki M, Yada N, Sofue K, Hyodo T, Onoda M, et al. Magnetic resonance elastography in the assessment of hepatic fibrosis: a study comparing transient elastography and histological data in the same patients. Abdom Radiol (NY) 2017;42:1659-1666.

142. Chen J, Yin M, Talwalkar JA, Oudry J, Glaser KJ, Smyrk TC, et al. Diagnostic performance of MR elastography and vibration-controlled transient elastography in the detection of hepatic fibrosis in patients with severe to morbid obesity. Radiology 2017;283:418428.

143. Korean Liver Cancer Association; National Cancer Center. 2018 Korean Liver Cancer Association-National Cancer Center Korea Practice Guidelines for the management of hepatocellular carcinoma. Gut Liver 2019;13:227-299.

144. Han KH, Kim DY, Park JY, Ahn SH, Kim J, Kim SU, et al. Survival of hepatocellular carcinoma patients may be improved in surveillance interval not more than 6 months compared with more than 6 months: a 15-year prospective study. J Clin Gastroenterol 2013;47:538-544.

145. Kim HY, Nam JY, Lee JH, Lee HA, Chang Y, Lee HY, et al. Intensity of surveillance for hepatocellular carcinoma determines survival in patients at risk in a hepatitis B-endemic area. Aliment Pharmacol Ther 2018:47:1490-1501.

146. Kim WR, Loomba R, Berg T, Aguilar Schall RE, Yee LJ, Dinh PV, et al. Impact of long-term tenofovir disoproxil fumarate on incidence of hepatocellular carcinoma in patients with chronic hepatitis B. Cancer 2015;121:3631-3638.

147. Liaw YF, Sheen IS, Lee CM, Akarca US, Papatheodoridis GV, SuetHing Wong $F$, et al. Tenofovir disoproxil fumarate (TDF), emtricitabine/TDF, and entecavir in patients with decompensated chronic hepatitis B liver disease. Hepatology 2011;53:62-72.

148. Liaw YF, Sung JJ, Chow WC, Farrell G, Lee CZ, Yuen H, et al. Lamivudine for patients with chronic hepatitis $B$ and advanced liver disease. N Engl J Med 2004;351:1521-1531.

149. Marcellin P, Gane E, Buti M, Afdhal N, Sievert W, Jacobson IM, et al. Regression of cirrhosis during treatment with tenofovir disoproxil fumarate for chronic hepatitis B: a 5-year open-label followup study. Lancet 2013;381:468-475. 
150. Matsumoto A, Tanaka E, Rokuhara A, Kiyosawa K, Kumada H, Omata $M$, et al. Efficacy of lamivudine for preventing hepatocellular carcinoma in chronic hepatitis B: a multicenter retrospective study of 2795 patients. Hepatol Res 2005;32:173-184.

151. Shim JH, Lee HC, Kim KM, Lim YS, Chung YH, Lee YS, et al. Efficacy of entecavir in treatment-naïve patients with hepatitis $B$ virusrelated decompensated cirrhosis. J Hepatol 2010;52:176-182.

152. Yuen MF, Seto WK, Chow DH, Tsui K, Wong DK, Ngai VW, et al. Long-term lamivudine therapy reduces the risk of long-term complications of chronic hepatitis B infection even in patients without advanced disease. Antivir Ther 2007;12:1295-1303.

153. Calvaruso V, Craxi A. Fibrosis in chronic viral hepatitis. Best Pract Res Clin Gastroenterol 2011;25:219-230.

154. Kim GA, Lim YS, An J, Lee D, Shim JH, Kim KM, et al. HBsAg seroclearance after nucleoside analogue therapy in patients with chronic hepatitis B: clinical outcomes and durability. Gut 2014;63:13251332.

155. Lok AS, McMahon BJ, Brown RS Jr, Wong JB, Ahmed AT, Farah $W$, et al. Antiviral therapy for chronic hepatitis B viral infection in adults: a systematic review and meta-analysis. Hepatology 2016;63:284-306.

156. Tang LSY, Covert E, Wilson E, Kottilil S. Chronic hepatitis B infection: a review. JAMA 2018;319:1802-1813.

157. Andreani T, Serfaty L, Mohand D, Dernaika S, Wendum D, Chazouillères 0 , et al. Chronic hepatitis B virus carriers in the immunotolerant phase of infection: histologic findings and outcome. Clin Gastroenterol Hepatol 2007;5:636-641.

158. Wong GL. Management of chronic hepatitis B patients in immunetolerant phase: what latest guidelines recommend. Clin Mol Hepatol 2018;24:108-113.

159. Kim GA, Lim YS, Han S, Choi J, Shim JH, Kim KM, et al. High risk of hepatocellular carcinoma and death in patients with immunetolerant-phase chronic hepatitis B. Gut 2018;67:945-952.

160. Kim MN, Kim SU, Kim BK, Park JY, Kim DY, Ahn SH, et al. Increased risk of hepatocellular carcinoma in chronic hepatitis B patients with transient elastography-defined subclinical cirrhosis. Hepatology 2015;61:1851-1859.

161. Sinn DH, Lee JH, Kim K, Ahn JH, Lee JH, Kim JH, et al. A novel model for predicting hepatocellular carcinoma development in patients with chronic hepatitis $B$ and normal alanine aminotransferase levels. Gut Liver 2017;11:528-534.

162. Park JY, Park YN, Kim DY, Paik YH, Lee KS, Moon BS, et al. High prevalence of significant histology in asymptomatic chronic hepatitis B patients with genotype C and high serum HBV DNA levels. J Viral Hepat 2008;15:615-621.

163. Chan HL, Chan CK, Hui AJ, Chan S, Poordad F, Chang TT, et al. Effects of tenofovir disoproxil fumarate in hepatitis $B$ e antigenpositive patients with normal levels of alanine aminotransfer- ase and high levels of hepatitis B virus DNA. Gastroenterology 2014;146:1240-1248.

164. Wong VW, Hui AJ, Wong GL, Chan RS, Chim AM, Lo AO, et al. Four-year outcomes after cessation of tenofovir in immune-tolerant chronic hepatitis B patients. J Clin Gastroenterol 2018;52:347-352.

165. Chang Y, Choe WH, Sinn DH, Lee JH, Ahn SH, Lee H, et al. Nucleos(t)ide analogue treatment for patients with hepatitis $B$ virus (HBV) e antigen-positive chronic HBV genotype C infection: a nationwide, multicenter, retrospective study. J Infect Dis 2017;216:1407-1414.

166. Cho JY, Paik YH, Sohn W, Cho HC, Gwak GY, Choi MS, et al. Patients with chronic hepatitis $B$ treated with oral antiviral therapy retain a higher risk for HCC compared with patients with inactive stage disease. Gut 2014;63:1943-1950.

167. Choi J, Han S, Kim N, Lim YS. Increasing burden of liver cancer despite extensive use of antiviral agents in a hepatitis $B$ virusendemic population. Hepatology 2017;66:1454-1463.

168. Yuen MF, Tanaka Y, Fong DY, Fung J, Wong DK, Yuen JC, et al. Independent risk factors and predictive score for the development of hepatocellular carcinoma in chronic hepatitis B. J Hepatol 2009;50:80-88.

169. Kwo PY, Cohen SM, Lim JK. ACG Clinical Guideline: evaluation of abnormal liver chemistries. Am J Gastroenterol 2017;112:18-35.

170. Lee JK, Shim JH, Lee HC, Lee SH, Kim KM, Lim YS, et al. Estimation of the healthy upper limits for serum alanine aminotransferase in Asian populations with normal liver histology. Hepatology 2010;51:1577-1583.

171. Chung WG, Kim HJ, Choe YG, Seok HS, Chon CW, Cho YK, et al. Clinical impacts of hazardous alcohol use and obesity on the outcome of entecavir therapy in treatment-naïve patients with chronic hepatitis B infection. Clin Mol Hepatol 2012;18:195-202.

172. Chao DT, Lim JK, Ayoub WS, Nguyen LH, Nguyen MH. Systematic review with meta-analysis: the proportion of chronic hepatitis $B$ patients with normal alanine transaminase $\leq 40 \mathrm{IU} / \mathrm{L}$ and significant hepatic fibrosis. Aliment Pharmacol Ther 2014;39:349-358.

173. Chen $C F$, Lee WC, Yang HI, Chang HC, Jen CL, Iloeje UH, et al. Changes in serum levels of HBV DNA and alanine aminotransferase determine risk for hepatocellular carcinoma. Gastroenterology 2011;141:1240-1248, 1248.e1-e2.

174. Yuen MF, Yuan HJ, Wong DK, Yuen JC, Wong WM, Chan AO, et al. Prognostic determinants for chronic hepatitis B in Asians: therapeutic implications. Gut 2005;54:1610-1614.

175. Park HN, Sinn DH, Gwak GY, Kim JE, Rhee SY, Eo SJ, et al. Upper normal threshold of serum alanine aminotransferase in identifying individuals at risk for chronic liver disease. Liver Int 2012;32:937944.

176. Chang TT, Gish RG, de Man R, Gadano A, Sollano J, Chao YC, et al. A comparison of entecavir and lamivudine for HBeAg-positive 
chronic hepatitis B. N Engl J Med 2006;354:1001-1010.

177. Hadziyannis SJ, Tassopoulos NC, Heathcote EJ, Chang TT, Kitis $G$, Rizzetto $M$, et al. Adefovir dipivoxil for the treatment of hepatitis B e antigen-negative chronic hepatitis B. N Engl J Med 2003;348:800-807.

178. Lai CL, Gane E, Liaw YF, Hsu CW, Thongsawat S, Wang Y, et al. Telbivudine versus lamivudine in patients with chronic hepatitis $B$. N Engl J Med 2007;357:2576-2588.

179. Lai CL, Shouval D, Lok AS, Chang TT, Cheinquer H, Goodman $Z$, et al. Entecavir versus lamivudine for patients with $\mathrm{HBeAg}$-negative chronic hepatitis B. N Engl J Med 2006;354:1011-1020.

180. Marcellin P, Chang TT, Lim SG, Tong MJ, Sievert W, Shiffman ML, et al. Adefovir dipivoxil for the treatment of hepatitis $B$ e antigenpositive chronic hepatitis B. N Engl J Med 2003;348:808-816.

181. Marcellin P, Heathcote EJ, Buti M, Gane E, de Man RA, Krastev Z, et al. Tenofovir disoproxil fumarate versus adefovir dipivoxil for chronic hepatitis B. N Engl J Med 2008;359:2442-2455.

182. Rockey DC, Caldwell SH, Goodman ZD, Nelson RC, Smith AD; American Association for the Study of Liver Diseases. Liver biopsy. Hepatology 2009:49:1017-1044.

183. European Association for Study of Liver; Asociacion Latinoamericana para el Estudio del Higado. EASL-ALEH Clinical Practice Guidelines: non-invasive tests for evaluation of liver disease severity and prognosis. J Hepatol 2015;63:237-264.

184. Korean Association for the Study of the Liver. KASL clinical practice guidelines: management of chronic hepatitis B. Clin Mol Hepatol 2016:22:18-75.

185. Song BC, Cho YK, Jwa H, Choi EK, Kim HU, Song HJ, et al. Is it necessary to delay antiviral therapy for 3-6 months to anticipate $\mathrm{HBeAg}$ seroconversion in patients with $\mathrm{HBeAg}$-positive chronic hepatitis B in endemic areas of HBV genotype C? Clin Mol Hepatol 2014;20:355-360.

186. Kim HS, Kim HJ, Shin WG, Kim KH, Lee JH, Kim HY, et al. Predictive factors for early $\mathrm{HBeAg}$ seroconversion in acute exacerbation of patients with HBeAg-positive chronic hepatitis B. Gastroenterology 2009;136:505-512.

187. Huang KW, Tam KW, Luo JC, Kuan YC. Efficacy and safety of lamivudine versus entecavir for treating chronic hepatitis B virus-related acute exacerbation and acute-on-chronic liver failure: a systematic review and meta-analysis. J Clin Gastroenterol 2017;51:539-547.

188. Tsai WL, Sun WC, Cheng JS. Chronic hepatitis B with spontaneous severe acute exacerbation. Int J Mol Sci 2015;16:28126-28145.

189. Chen CH, Lee CM, Lu SN, Wang JH, Tung HD, Hung CH, et al. Comparison of clinical outcome between patients continuing and discontinuing lamivudine therapy after biochemical breakthrough of YMDD mutants. J Hepatol 2004;41:454-461.

190. Kim KH, Sinn DH, Yun WK, Cho HC, Lee YY, Gwak GY, et al. Defining virologic relapse in chronic hepatitis B. Dig Dis Sci
2011;56:2432-2438.

191. Shih CA, Chen WC, Yu HC, Cheng JS, Lai KH, Hsu JT, et al. Risk of severe acute exacerbation of chronic HBV infection cancer patients who underwent chemotherapy and did not receive anti-viral prophylaxis. PLoS One 2015;10:e0132426.

192. Garg H, Sarin SK, Kumar M, Garg V, Sharma BC, Kumar A. Tenofovir improves the outcome in patients with spontaneous reactivation of hepatitis B presenting as acute-on-chronic liver failure. Hepatology 2011;53:774-780.

193. Tsai WL, Chiang PH, Chan HH, Lin HS, Lai KH, Cheng JS, et al. Early entecavir treatment for chronic hepatitis B with severe acute exacerbation. Antimicrob Agents Chemother 2014;58:1918-1921.

194. Wong VW, Wong GL, Yiu KK, Chim AM, Chu SH, Chan HY, et al. Entecavir treatment in patients with severe acute exacerbation of chronic hepatitis B. J Hepatol 2011;54:236-242.

195. Ha JM, Sohn W, Cho JY, Pyo JH, Choi K, Sinn DH, et al. Static and dynamic prognostic factors for hepatitis-B-related acute-on-chronic liver failure. Clin Mol Hepatol 2015;21:232-241.

196. Park JG, Lee YR, Park SY, Lee HJ, Tak WY, Kweon YO, et al. Tenofovir, entecavir, and lamivudine in patients with severe acute exacerbation and hepatic decompensation of chronic hepatitis B. Dig Liver Dis 2018;50:163-167.

197. Chen CH, Lin CL, Hu TH, Hung CH, Tseng PL, Wang JH, et al. Entecavir vs. lamivudine in chronic hepatitis $B$ patients with severe acute exacerbation and hepatic decompensation. J Hepatol 2014;60:1127-1134.

198. Yasui S, Fujiwara K, Nakamura M, Miyamura T, Yonemitsu Y, Mikata $R$, et al. Virological efficacy of combination therapy with corticosteroid and nucleoside analogue for severe acute exacerbation of chronic hepatitis B. J Viral Hepat 2015;22:94-102.

199. Yue-Meng $W$, Yang $L H$, Yang JH, Xu Y, Yang J, Song GB. The effect of plasma exchange on entecavir-treated chronic hepatitis $B$ patients with hepatic de-compensation and acute-on-chronic liver failure. Hepatol Int 2016;10:462-469.

200. Ahn JM, Sinn DH, Gwak GY, Paik YH, Choi MS, Lee JH, et al. Prediction of clinical outcomes in hepatitis $B$ e antigen negative chronic hepatitis B patients with elevated hepatitis B virus DNA levels. PLoS One 2015;10:e0144777.

201. Paik N, Sinn DH, Lee JH, Oh IS, Kim JH, Kang W, et al. Non-invasive tests for liver disease severity and the hepatocellular carcinoma risk in chronic hepatitis B patients with low-level viremia. Liver Int 2018;38:68-75.

202. de Niet A, Jansen L, Stelma F, Willemse SB, Kuiken SD, Weijer S, et al. Peg-interferon plus nucleotide analogue treatment versus no treatment in patients with chronic hepatitis B with a low viral load: a randomised controlled, open-label trial. Lancet Gastroenterol Hepatol 2017;2:576-584.

203. Chang TT, Liaw YF, Wu SS, Schiff E, Han KH, Lai CL, et al. Long- 
term entecavir therapy results in the reversal of fibrosis/cirrhosis and continued histological improvement in patients with chronic hepatitis B. Hepatology 2010;52:886-893.

204. Lee J, Sinn DH, Kim JH, Gwak GY, Kim HS, Jung SH, et al. Hepatocellular carcinoma risk of compensated cirrhosis patients with elevated HBV DNA levels according to serum aminotransferase levels. J Korean Med Sci 2015;30:1618-1624.

205. Kim JH, Sinn DH, Kang W, Gwak GY, Paik YH, Choi MS, et al. Lowlevel viremia and the increased risk of hepatocellular carcinoma in patients receiving entecavir treatment. Hepatology 2017;66:335343.

206. Sinn DH, Lee J, Goo J, Kim K, Gwak GY, Paik YH, et al. Hepatocellular carcinoma risk in chronic hepatitis $B$ virus-infected compensated cirrhosis patients with low viral load. Hepatology 2015;62:694701.

207. Cho YY, Lee JH, Chang Y, Nam JY, Cho H, Lee DH, et al. Comparison of overall survival between antiviral-induced viral suppression and inactive phase chronic hepatitis B patients. J Viral Hepat 2018;25:1161-1171.

208. Suk KT, Baik SK, Yoon JH, Cheong JY, Paik YH, Lee CH, et al. Revision and update on clinical practice guideline for liver cirrhosis. Korean J Hepatol 2012;18:1-21.

209. Jang JW, Choi JY, Kim YS, Woo HY, Choi SK, Lee CH, et al. Longterm effect of antiviral therapy on disease course after decompensation in patients with hepatitis B virus-related cirrhosis. Hepatology 2015;61:1809-1820.

210. Fontana RJ, Hann HW, Perrillo RP, Vierling JM, Wright T, Rakela J, et al. Determinants of early mortality in patients with decompensated chronic hepatitis B treated with antiviral therapy. Gastroenterology 2002;123:719-727.

211. Perrillo R, Tamburro C, Regenstein F, Balart L, Bodenheimer $H$, Silva $M$, et al. Low-dose, titratable interferon alfa in decompensated liver disease caused by chronic infection with hepatitis B virus. Gastroenterology 1995;109:908-916.

212. Kim YJ, Cho HC, Choi MS, Lee JH, Koh KC, Yoo BC, et al. The change of the quantitative $\mathrm{HBsAg}$ level during the natural course of chronic hepatitis B. Liver Int 2011;31:817-823.

213. Wang L, Zou ZQ, Wang K, Yu JG, Liu XZ. Role of serum hepatitis $B$ virus marker quantitation to differentiate natural history phases of HBV infection. Hepatol Int 2016;10:133-138.

214. Yim SY, Um SH, Jung JY, Seo YS, Yim HJ, Ryu HS, et al. Role of hepatitis $B$ surface antigen ( $\mathrm{HBSAg}$ ) in identifying true inactive HBsAg carriers infected with genotype C hepatitis B virus. J Clin Gastroenterol 2014;48:166-171.

215. Koumbi L. Current and future antiviral drug therapies of hepatitis B chronic infection. World J Hepatol 2015;7:1030-1040.

216. Lau GK, Piratvisuth T, Luo KX, Marcellin P, Thongsawat S, Cooksley $\mathrm{G}$, et al. Peginterferon Alfa-2a, lamivudine, and the combination for
HBeAg-positive chronic hepatitis B. N Engl J Med 2005;352:26822695.

217. Marcellin P, Lau GK, Bonino F, Farci $P$, Hadziyannis $S$, Jin $R$, et al. Peginterferon alfa-2a alone, lamivudine alone, and the two in combination in patients with $\mathrm{HBeAg}$-negative chronic hepatitis $\mathrm{B}$. N Engl J Med 2004;351:1206-1217.

218. Qiu K, Liu B, Li SY, Li H, Chen ZW, Luo AR, et al. Systematic review with meta-analysis: combination treatment of regimens based on pegylated interferon for chronic hepatitis $B$ focusing on hepatitis $B$ surface antigen clearance. Aliment Pharmacol Ther 2018;47:13401348.

219. Viganò M, Grossi G, Loglio A, Lampertico P. Treatment of hepatitis B: is there still a role for interferon? Liver Int 2018;38 Suppl 1:79-83.

220. Janssen HL, van Zonneveld M, Senturk H, Zeuzem S, Akarca US, Cakaloglu $Y$, et al. Pegylated interferon alfa-2b alone or in combination with lamivudine for $\mathrm{HBeAg}$-positive chronic hepatitis $\mathrm{B}$ : a randomised trial. Lancet 2005;365:123-129.

221. Li WC, Wang MR, Kong LB, Ren WG, Zhang YG, Nan YM. Peginterferon alpha-based therapy for chronic hepatitis $B$ focusing on $\mathrm{HBsAg}$ clearance or seroconversion: a meta-analysis of controlled clinical trials. BMC Infect Dis 2011;11:165.

222. Zoulim F, Locarnini S. Hepatitis B virus resistance to nucleos(t)ide analogues. Gastroenterology 2009;137:1593-1608.e1-e2.

223. An J, Lim YS, Kim GA, Han SB, Jeong W, Lee D, et al. Telbivudine versus entecavir in patients with undetectable hepatitis $B$ virus DNA: a randomized trial. BMC Gastroenterol 2017;17:15.

224. Agarwal K, Brunetto M, Seto WK, Lim YS, Fung S, Marcellin P, et al. 96 weeks treatment of tenofovir alafenamide vs. tenofovir disoproxil fumarate for hepatitis B virus infection. J Hepatol 2018;68:672-681.

225. Chan HL, Fung S, Seto WK, Chuang WL, Chen CY, Kim HJ, et al. Tenofovir alafenamide versus tenofovir disoproxil fumarate for the treatment of $\mathrm{HBeAg}$-positive chronic hepatitis B virus infection: a randomised, double-blind, phase 3, non-inferiority trial. Lancet Gastroenterol Hepatol 2016;1:185-195.

226. Buti M, Gane E, Seto WK, Chan HL, Chuang WL, Stepanova T, et al. Tenofovir alafenamide versus tenofovir disoproxil fumarate for the treatment of patients with $\mathrm{HBeAg}$-negative chronic hepatitis B virus infection: a randomised, double-blind, phase 3, non-inferiority trial. Lancet Gastroenterol Hepatol 2016;1:196-206.

227. Seto WK, Asahina Y, Brown TT, Peng CY, Stanciu C, Abdurakhmanov $D$, et al. Improved bone safety of tenofovir alafenamide compared to tenofovir disoproxil fumarate over 2 years in patients with chronic HBV infection. Clin Gastroenterol Hepatol. 2018 Jun 20. pii: S1542-3565(18)30633-5. doi: 10.1016/j.cgh.2018.06.023.

228. Yuen MF, Kim J, Kim CR, Ngai V, Yuen JC, Min C, et al. A randomized placebo-controlled, dose-finding study of oral LB80380 in HBeAg-positive patients with chronic hepatitis B. Antivir Ther 
2006;11:977-983.

229. Yuen MF, Lee SH, Kang HM, Kim CR, Kim J, Ngai V, et al. Pharmacokinetics of LB80331 and LB80317 following oral administration of LB80380, a new antiviral agent for chronic hepatitis B (CHB), in healthy adult subjects, CHB patients, and mice. Antimicrob Agents Chemother 2009;53:1779-1785.

230. Ahn SH, Kim W, Jung YK, Yang JM, Jang JY, Kweon YO, et al. Safety and efficacy of besifovir in treatment-naïve chronic hepatitis $B$ virus infection: a randomized, double-blind, double dummy, phase 3 study [Abstract]. J Hepatol 2017;66:S88-S89.

231. Lam YF, Seto WK, Wong D, Cheung KS, Fung J, Mak LY, et al. Seven-year treatment outcome of entecavir in a real-world cohort: effects on clinical parameters, HBsAg and HBcrAg levels. Clin Transl Gastroenterol 2017;8:e125.

232. Hou J, Zhao W, Lee C, Hann HW, Peng CY, Tanwandee T, et al. Prospective, randomized assessment of $\mathrm{HBV}$-associated and other clinical outcome events during long-term therapy with entecavir or other HBV nucleos(t)ide analogues in patients with chronic HBV infection [Abstract]. Hepatology 2017;66(S1):12A-13A.

233. Liu Y, Corsa AC, Buti $M$, Cathcart AL, Flaherty JF, Miller MD, et al. No detectable resistance to tenofovir disoproxil fumarate in $\mathrm{HBeAg}+$ and $\mathrm{HBeAg}$ - patients with chronic hepatitis B after 8 years of treatment. J Viral Hepat 2017;24:68-74.

234. Konerman MA, Lok AS. Interferon treatment for hepatitis B. Clin Liver Dis 2016;20:645-665.

235. Cooksley WG, Piratvisuth T, Lee SD, Mahachai V, Chao YC, Tanwandee $T$, et al. Peginterferon alpha-2a (40 kDa): an advance in the treatment of hepatitis B e antigen-positive chronic hepatitis $B$. J Viral Hepat 2003;10:298-305.

236. Chan HL, Leung NW, Hui AY, Wong VW, Liew CT, Chim AM, et al. A randomized, controlled trial of combination therapy for chronic hepatitis B: comparing pegylated interferon-alpha2b and lamivudine with lamivudine alone. Ann Intern Med 2005;142:240-250.

237. Buster EH, Flink HJ, Cakaloglu Y, Simon K, Trojan J, Tabak F, et al. Sustained $\mathrm{HBeAg}$ and $\mathrm{HBsAg}$ loss after long-term follow-up of HBeAg-positive patients treated with peginterferon alpha-2b. Gastroenterology 2008;135:459-467.

238. Liaw YF, Jia JD, Chan HL, Han KH, Tanwandee T, Chuang WL, et al. Shorter durations and lower doses of peginterferon alfa-2a are associated with inferior hepatitis $B$ e antigen seroconversion rates in hepatitis B virus genotypes B or C. Hepatology 2011;54:1591-1599.

239. Cho JY, Sohn W, Sinn DH, Gwak GY, Paik YH, Choi MS, et al. Longterm real-world entecavir therapy in treatment-naïve hepatitis $B$ patients: base-line hepatitis B virus DNA and hepatitis B surface antigen levels predict virologic response. Korean J Intern Med 2017;32:636-646.

240. Cai S, Li Z, Yu T, Xia M, Peng J. Serum hepatitis B core antibody levels predict $\mathrm{HBeAg}$ seroconversion in chronic hepatitis $B$ patients with high viral load treated with nucleos(t)ide analogs. Infect Drug Resist 2018;11:469-477.

241. Song $G$, Yang R, Rao $H$, Feng B, Ma $H$, Jin Q, et al. Serum HBV core-related antigen is a good predictor for spontaneous $\mathrm{HBeAg}$ seroconversion in chronic hepatitis B patients. J Med Virol 2017;89:463-468.

242. Yang J, Chen J, Ye P, Jin L, Wu W, Sheng G, et al. HBsAg as an important predictor of $\mathrm{HBeAg}$ seroconversion following antiviral treatment for HBeAg-positive chronic hepatitis B patients. J Transl Med 2014;12:183.

243. You H, Ma H, Liu T, Cong M, Wang P, Ou X, et al. Different models of $\mathrm{HBeAg}$ seroconversion predicated by on-treatment ALT and HBV DNA profiles. J Viral Hepat 2009;16:876-882.

244. Zeuzem S, Gane E, Liaw YF, Lim SG, DiBisceglie A, Buti M, et al. Baseline characteristics and early on-treatment response predict the outcomes of 2 years of telbivudine treatment of chronic hepatitis B. J Hepatol 2009;51:11-20.

245. Gane EJ. The Roadmap concept: using early on-treatment virologic responses to optimize long-term outcomes for patients with chronic hepatitis B. Hepatol Int 2008;2:304-307.

246. Chan HL, Wong VW, Tse CH, Chim AM, Chan HY, Wong GL, et al. Early virological suppression is associated with good maintained response to adefovir dipivoxil in lamivudine resistant chronic hepatitis B. Aliment Pharmacol Ther 2007;25:891-898.

247. Yuen MF, Fong DY, Wong DK, Yuen JC, Fung J, Lai CL. Hepatitis $B$ virus DNA levels at week 4 of lamivudine treatment predict the 5-year ideal response. Hepatology 2007:46:1695-1703.

248. Gish RG, Chang TT, Lai CL, de Man R, Gadano A, Poordad F, et al. Loss of HBsAg antigen during treatment with entecavir or lamivudine in nucleoside-naïve $\mathrm{HBeAg-positive} \mathrm{patients} \mathrm{with} \mathrm{chronic}$ hepatitis B. J Viral Hepat 2010;17:16-22.

249. Marcellin P, Buti M, Krastev Z, de Man RA, Zeuzem S, Lou L, et al. Kinetics of hepatitis $B$ surface antigen loss in patients with $\mathrm{HBeAg}$ positive chronic hepatitis $B$ treated with tenofovir disoproxil fumarate. J Hepatol 2014;61:1228-1237.

250. Fung S, Gordon SC, Krastev Z, Horban A, Petersen J, Sperl J, et al. Tenofovir disoproxil fumarate in Asian or Pacific Islander chronic hepatitis B patients with high viral load ( $\geq 9 \log 10$ copies/ml). Liver Int 2015;35:422-428.

251. Buster EH, Hansen BE, Lau GK, Piratvisuth T, Zeuzem S, Steyerberg $E W$, et al. Factors that predict response of patients with hepatitis $B$ e antigen-positive chronic hepatitis B to peginterferon-alfa. Gastroenterology 2009;137:2002-2009.

252. Kong LN, Qin B, Ma Q, Li L, Yao Y. Relationship between hepatitis $B$ virus genotype $B$ and $C$ and response to interferon therapy in HBeAg positive chronic hepatitis B patients: a meta-analysis. J Gastroenterol Hepatol 2014;29:1387-1395.

253. Chan HLY, Messinger D, Papatheodoridis GV, Cornberg M, Xie Q, 
Piratvisuth $T$, et al. A baseline tool for predicting response to peginterferon alfa-2a in HBeAg-positive patients with chronic hepatitis B. Aliment Pharmacol Ther 2018;48:547-555.

254. Goulis I, Karatapanis S, Akriviadis E, Deutsch M, Dalekos GN, Raptopoulou-Gigi M, et al. On-treatment prediction of sustained response to peginterferon alfa-2a for $\mathrm{HBeAg}$-negative chronic hepatitis B patients. Liver Int 2015;35:1540-1548.

255. Moucari R, Mackiewicz V, Lada O, Ripault MP, Castelnau C, Martinot-Peignoux $\mathrm{M}$, et al. Early serum HBsAg drop: a strong predictor of sustained virological response to pegylated interferon alfa-2a in HBeAg-negative patients. Hepatology 2009;49:1151-1157.

256. Piratvisuth T, Marcellin P, Popescu M, Kapprell HP, Rothe V, Lu ZM. Hepatitis $B$ surface antigen: association with sustained response to peginterferon alfa-2a in hepatitis $B$ e antigen-positive patients. Hepatol Int 2013;7:429-436.

257. Locarnini S, Mason WS. Cellular and virological mechanisms of HBV drug resistance. J Hepatol 2006;44:422-431.

258. Yuan HJ, Yuen MF, Ka-Ho Wong D, Sablon E, Lai CL. The relationship between HBV-DNA levels and cirrhosis-related complications in Chinese with chronic hepatitis B. J Viral Hepat 2005;12:373-379.

259. Jung YK, Kim JH, Lee YS, Lee HJ, Yoon E, Jung ES, et al. Change in serum hepatitis $B$ surface antigen level and its clinical significance in treatment-naïve, hepatitis B e antigen-positive patients receiving entecavir. J Clin Gastroenterol 2010;44:653-657.

260. Manesis EK, Hadziyannis ES, Angelopoulou OP, Hadziyannis SJ. Prediction of treatment-related HBsAg loss in HBeAG-negative chronic hepatitis B: a clue from serum HBsAg levels. Antivir Ther 2007;12:73-82.

261. Reijnders JG, Rijckborst V, Sonneveld MJ, Scherbeijn SM, Boucher $C A$, Hansen BE, et al. Kinetics of hepatitis B surface antigen differ between treatment with peginterferon and entecavir. J Hepatol 2011;54:449-454.

262. Gramenzi A, Loggi E, Micco L, Cursaro C, Fiorino S, Galli S, et al. Serum hepatitis $B$ surface antigen monitoring in long-term lamivudine-treated hepatitis B virus patients. J Viral Hepat 2011;18:e468e474.

263. Kwon JH, Jang JW, Lee S, Lee J, Chung KW, Lee YS, et al. Pretreatment $\mathrm{HBeAg}$ level and an early decrease in HBeAg level predict virologic response to entecavir treatment for $\mathrm{HBeAg}$-positive chronic hepatitis B. J Viral Hepat 2012;19:e41-e47.

264. Zoulim F, Carosi G, Greenbloom S, Mazur W, Nguyen T, Jeffers L, et al. Quantification of HBsAg in nucleos(t)ide-naïve patients treated for chronic hepatitis $B$ with entecavir with or without tenofovir in the BE-LOW study. J Hepatol 2015;62:56-63.

265. Seto WK, Wong DK, Fung J, Huang FY, Lai CL, Yuen MF. Reduction of hepatitis $B$ surface antigen levels and hepatitis $B$ surface antigen seroclearance in chronic hepatitis B patients receiving 10 years of nucleoside analogue therapy. Hepatology 2013;58:923-931.
266. Chen $\mathrm{CH}$, Hung CH, Hu TH, Wang JH, Lu SN, Su PF, et al. Association between level of hepatitis $B$ surface antigen and relapse after entecavir therapy for chronic hepatitis B virus infection. Clin Gastroenterol Hepatol 2015;13:1984-1992.e1.

267. Chen CH, Lu SN, Hung CH, Wang JH, Hu TH, Changchien CS, et al. The role of hepatitis $B$ surface antigen quantification in predicting $\mathrm{HBsAg}$ loss and HBV relapse after discontinuation of lamivudine treatment. J Hepatol 2014;61:515-522.

268. Hsu YC, Mo LR, Chang CY, Wu MS, Kao JH, Wang WL, et al. Association between serum level of hepatitis $B$ surface antigen at end of entecavir therapy and risk of relapse in $E$ antigen-negative patients. Clin Gastroenterol Hepatol 2016;14:1490-1498.e3.

269. Wang CC, Tseng KC, Hsieh TY, Tseng TC, Lin HH, Kao JH. Assessing the durability of entecavir-treated hepatitis B using quantitative HBsAg. Am J Gastroenterol 2016;111:1286-1294.

270. Kim HS, Han KH, Ahn SH, Kim EO, Chang HY, Moon MS, et al. Evaluation of methods for monitoring drug resistance in chronic hepatitis B patients during lamivudine therapy based on mass spectrometry and reverse hybridization. Antivir Ther 2005;10:441449.

271. Lee JM, Kim HJ, Park JY, Lee CK, Kim DY, Kim JK, et al. Rescue monotherapy in lamivudine-resistant hepatitis B e antigenpositive chronic hepatitis B: adefovir versus entecavir. Antivir Ther 2009;14:705-712.

272. Lee JM, Park JY, Kim DY, Nguyen T, Hong SP, Kim SO, et al. Longterm adefovir dipivoxil monotherapy for up to 5 years in lamivudine-resistant chronic hepatitis B. Antivir Ther 2010;15:235-241.

273. Park S, Kim WI, Cho DH, Kim YJ, Kim HS, Kim JH, et al. Adefovirinduced Fanconi syndrome associated with osteomalacia. Clin Mol Hepatol 2017;24:339-344.

274. Samarkos M, Theofanis V, Eliadi I, Vlachogiannakos J, Polyzos A. Tenofovir-associated Fanconi syndrome in a patient with chronic hepatitis B. J Gastrointestin Liver Dis 2014;23:342.

275. Fleischer RD, Lok AS. Myopathy and neuropathy associated with nucleos(t)ide analog therapy for hepatitis B. J Hepatol 2009;51:787791.

276. Kim BK, Oh J, Kwon SY, Choe WH, Ko SY, Rhee KH, et al. Clevudine myopathy in patients with chronic hepatitis B. J Hepatol 2009;51:829-834

277. Tak WY, Park SY, Cho CM, Jung MK, Jeon SW, Kweon YO, et al. Clinical, biochemical, and pathological characteristics of clevudineassociated myopathy. J Hepatol 2010;53:261-266.

278. Brunetto MR, Moriconi F, Bonino F, Lau GK, Farci P, Yurdaydin C, et al. Hepatitis $B$ virus surface antigen levels: a guide to sustained response to peginterferon alfa-2a in $\mathrm{HBeAg-negative} \mathrm{chronic} \mathrm{hepa-}$ titis B. Hepatology 2009;49:1141-1150.

279. Marcellin P, Bonino F, Yurdaydin C, Hadziyannis S, Moucari R, Kapprell HP, et al. Hepatitis B surface antigen levels: association with 
5-year response to peginterferon alfa-2a in hepatitis B e-antigennegative patients. Hepatol Int 2013;7:88-97.

280. Chen X, Chen X, Chen W, Ma X, Huang J, Chen R. Extended peginterferon alfa-2a (Pegasys) therapy in Chinese patients with $\mathrm{HBeAg}$ negative chronic hepatitis B. J Med Virol 2014;86:1705-1713.

281. Lampertico P, Viganò M, Di Costanzo GG, Sagnelli E, Fasano M, Di Marco $V$, et al. Randomised study comparing 48 and 96 weeks peginterferon a-2a therapy in genotype D HBeAg-negative chronic hepatitis B. Gut 2013;62:290-298.

282. Kang HS, Um SH, Seo YS, An H, Lee KG, Hyun JJ, et al. Healthy range for serum ALT and the clinical significance of "unhealthy" normal ALT levels in the Korean population. J Gastroenterol Hepatol 2011;26:292-299.

283. Fattovich G, Olivari N, Pasino M, D'Onofrio M, Martone E, Donato F. Long-term outcome of chronic hepatitis B in Caucasian patients: mortality after 25 years. Gut 2008;57:84-90.

284. Fattovich G, Pantalena M, Zagni I, Realdi G, Schalm SW, Christensen $E$, et al. Effect of hepatitis $B$ and $C$ virus infections on the natural history of compensated cirrhosis: a cohort study of 297 patients. Am J Gastroenterol 2002;97:2886-2895.

285. Mommeja-Marin H, Mondou E, Blum MR, Rousseau F. Serum HBV DNA as a marker of efficacy during therapy for chronic HBV infection: analysis and review of the literature. Hepatology 2003;37:1309-1319.

286. Hadziyannis SJ, Sevastianos V, Rapti I, Vassilopoulos D, Hadziyannis $\mathrm{E}$. Sustained responses and loss of HBsAg in HBeAg-negative patients with chronic hepatitis B who stop long-term treatment with adefovir. Gastroenterology 2012;143:629-636.e1.

287. Papatheodoridis G, Vlachogiannakos I, Cholongitas E, Wursthorn $K$, Thomadakis C, Touloumi $G$, et al. Discontinuation of oral antivirals in chronic hepatitis B: a systematic review. Hepatology 2016;63:1481-1492.

288. Berg T, Simon KG, Mauss S, Schott E, Heyne R, Klass DM, et al. Long-term response after stopping tenofovir disoproxil fumarate in non-cirrhotic HBeAg-negative patients - FINITE study. J Hepatol 2017;67:918-924.

289. Sarin SK, Kumar M, Lau GK, Abbas Z, Chan HL, Chen CJ, et al. Asian-Pacific clinical practice guidelines on the management of hepatitis B: a 2015 update. Hepatol Int 2016;10:1-98.

290. Jeng WJ, Sheen IS, Chen YC, Hsu CW, Chien RN, Chu CM, et al. Off-therapy durability of response to entecavir therapy in hepatitis $B$ e antigen-negative chronic hepatitis B patients. Hepatology 2013;58:1888-1896.

291. Kim YJ, Kim K, Hwang SH, Kim SS, Lee D, Cheong JY, et al. Durability after discontinuation of nucleos(t)ide therapy in chronic $\mathrm{HBeAg}$ negative hepatitis patients. Clin Mol Hepatol 2013;19:300-304.

292. Seto WK, Hui AJ, Wong VW, Wong GL, Liu KS, Lai CL, et al. Treatment cessation of entecavir in Asian patients with hepatitis $B$ e an- tigen negative chronic hepatitis B: a multicentre prospective study. Gut 2015;64:667-672.

293. Fattovich G, Rugge M, Brollo L, Pontisso P, Noventa F, Guido M, et al. Clinical, virologic and histologic outcome following seroconversion from $\mathrm{HBeAg}$ to anti-HBe in chronic hepatitis type B. Hepatology 1986;6:167-172.

294. Yang HI, Lu SN, Liaw YF, You SL, Sun CA, Wang LY, et al. Hepatitis $\mathrm{B}$ e antigen and the risk of hepatocellular carcinoma. N Engl J Med 2002;347:168-174.

295. Niederau C, Heintges T, Lange S, Goldmann G, Niederau CM, Mohr $\mathrm{L}$, et al. Long-term follow-up of HBeAg-positive patients treated with interferon alfa for chronic hepatitis B. N Engl J Med 1996;334:1422-1427.

296. Reijnders JG, Perquin MJ, Zhang N, Hansen BE, Janssen HL. Nucleos(t)ide analogues only induce temporary hepatitis B e antigen seroconversion in most patients with chronic hepatitis B. Gastroenterology 2010;139:491-498.

297. Song BC, Suh DJ, Lee HC, Chung YH, Lee YS. Hepatitis B e antigen seroconversion after lamivudine therapy is not durable in patients with chronic hepatitis B in Korea. Hepatology 2000;32(4 Pt 1):803806.

298. Byun KS, Kwon OS, Kim JH, Yim HJ, Chang YJ, Kim JY, et al. Factors related to post-treatment relapse in chronic hepatitis $B$ patients who lost HBeAg after lamivudine therapy. J Gastroenterol Hepatol 2005;20:1838-1842.

299. Lee HW, Lee HJ, Hwang JS, Sohn JH, Jang JY, Han KJ, et al. Lamivudine maintenance beyond one year after $\mathrm{HBeAg}$ seroconversion is a major factor for sustained virologic response in $\mathrm{HBeAg}$-positive chronic hepatitis B. Hepatology 2010;51:415-421.

300. Chan HL, Wong VW, Tse AM, Tse CH, Chim AM, Chan HY, et al. Serum hepatitis $B$ surface antigen quantitation can reflect hepatitis $B$ virus in the liver and predict treatment response. Clin Gastroenterol Hepatol 2007:5:1462-1468.

301. Kim JH, Lee YS, Lee HJ, Yoon E, Jung YK, Jong ES, et al. HBsAg seroclearance in chronic hepatitis $\mathrm{B}$ : implications for hepatocellular carcinoma. J Clin Gastroenterol 2011;45:64-68.

302. Yip TC, Wong GL, Wong VW, Tse YK, Lui GC, Lam KL, et al. Durability of hepatitis $B$ surface antigen seroclearance in untreated and nucleos(t)ide analogue-treated patients. J Hepatol. 2017 Oct 6. pii: S0168-8278(17)32332-2. doi: 10.1016/j.jhep.2017.09.018.

303. Chevaliez S, Hézode C, Bahrami S, Grare M, Pawlotsky JM. Longterm hepatitis $B$ surface antigen ( $\mathrm{HBs} A g$ ) kinetics during nucleoside/nucleotide analogue therapy: finite treatment duration unlikely. J Hepatol 2013;58:676-683.

304. Seto WK, Liu K, Wong DK, Fung J, Huang FY, Hung IF, et al. Patterns of hepatitis $B$ surface antigen decline and HBV DNA suppression in Asian treatment-experienced chronic hepatitis B patients after three years of tenofovir treatment. J Hepatol 2013;59:709- 
716.

305. Fung J, Cheung KS, Wong DK, Mak LY, To WP, Seto WK, et al. Long term outcomes and predictive scores for hepatocellular carcinoma and hepatitis B surface antigen seroclearance after hepatitis B eantigen seroclearance. Hepatology 2018;68:462-472.

306. Kim GA, Lee HC, Kim MJ, Ha Y, Park EJ, An J, et al. Incidence of hepatocellular carcinoma after HBsAg seroclearance in chronic hepatitis B patients: a need for surveillance. J Hepatol 2015;62:10921099.

307. Nafa S, Ahmed S, Tavan D, Pichoud C, Berby F, Stuyver L, et al. Early detection of viral resistance by determination of hepatitis $B$ virus polymerase mutations in patients treated by lamivudine for chronic hepatitis B. Hepatology 2000;32:1078-1088.

308. Zoulim F, Durantel D, Deny P. Management and prevention of drug resistance in chronic hepatitis B. Liver Int 2009;29 Suppl 1:108-115.

309. Lai CL, Dienstag J, Schiff E, Leung NW, Atkins M, Hunt C, et al. Prevalence and clinical correlates of YMDD variants during lamivudine therapy for patients with chronic hepatitis B. Clin Infect Dis 2003;36:687-696.

310. Melegari M, Scaglioni PP, Wands JR. Hepatitis B virus mutants associated with 3TC and famciclovir administration are replication defective. Hepatology 1998;27:628-633.

311. Tenney DJ, Levine SM, Rose RE, Walsh AW, Weinheimer SP, Discotto $L$, et al. Clinical emergence of entecavir-resistant hepatitis $B$ virus requires additional substitutions in virus already resistant to Lamivudine. Antimicrob Agents Chemother 2004;48:3498-3507.

312. Bartholomeusz A, Locarnini SA. Antiviral drug resistance: clinical consequences and molecular aspects. Semin Liver Dis 2006;26:162170.

313. Lok AS, Lai CL, Leung N, Yao GB, Cui ZY, Schiff ER, et al. Longterm safety of lamivudine treatment in patients with chronic hepatitis B. Gastroenterology 2003;125:1714-1722.

314. Lok AS, Zoulim F, Locarnini S, Bartholomeusz A, Ghany MG, Pawlotsky JM, et al. Antiviral drug-resistant HBV: standardization of nomenclature and assays and recommendations for management. Hepatology 2007;46:254-265.

315. Gish RG, Trinh H, Leung N, Chan FK, Fried MW, Wright TL, et al. Safety and antiviral activity of emtricitabine (FTC) for the treatment of chronic hepatitis B infection: a two-year study. J Hepatol 2005:43:60-66.

316. Stuyver LJ, Locarnini SA, Lok A, Richman DD, Carman WF, Dienstag $J \mathrm{~L}$, et al. Nomenclature for antiviral-resistant human hepatitis $B$ virus mutations in the polymerase region. Hepatology 2001;33:751757.

317. Liaw YF, Gane E, Leung N, Zeuzem S, Wang Y, Lai CL, et al. 2-Year GLOBE trial results: telbivudine is superior to lamivudine in patients with chronic hepatitis B. Gastroenterology 2009;136:486-495.

318. Koh KH, Kang CJ, Kim DH, Choi YW, Kim MJ, Cheong JY, et al.
Development of clevudine resistance after switching from lamivudine in a patient with chronic hepatitis B. Korean J Gastroenterol 2008:52:325-328

319. Yoon EL, Yim HJ, Lee HJ, Lee YS, Kim JH, Jung ES, et al. Comparison of clevudine and entecavir for treatment-naive patients with chronic hepatitis B virus infection: two-year follow-up data. J Clin Gastroenterol 2011;45:893-899.

320. Locarnini S. Molecular virology and the development of resistant mutants: implications for therapy. Semin Liver Dis 2005;25 Suppl $1: 9-19$

321. Qi X, Xiong S, Yang H, Miller M, Delaney WE 4th. In vitro susceptibility of adefovir-associated hepatitis $B$ virus polymerase mutations to other antiviral agents. Antivir Ther 2007;12:355-362.

322. Tenney DJ, Rose RE, Baldick CJ, Levine SM, Pokornowski KA, Walsh AW, et al. Two-year assessment of entecavir resistance in Lamivudine-refractory hepatitis B virus patients reveals different clinical outcomes depending on the resistance substitutions present. Antimicrob Agents Chemother 2007;51:902-911.

323. Tenney DJ, Rose RE, Baldick CJ, Pokornowski KA, Eggers BJ, Fang $J$, et al. Long-term monitoring shows hepatitis $B$ virus resistance to entecavir in nucleoside-naive patients is rare through 5 years of therapy. Hepatology 2009;49:1503-1514.

324. Lee JH, Cho Y, Lee DH, Lee M, Yoo JJ, Choi WM, et al. Prior exposure to lamivudine increases entecavir resistance risk in chronic hepatitis B patients without detectable lamivudine resistance. Antimicrob Agents Chemother 2014;58:1730-1737.

325. Hadziyannis SJ, Tassopoulos NC, Heathcote EJ, Chang TT, Kitis $\mathrm{G}$, Rizzetto $\mathrm{M}$, et al. Long-term therapy with adefovir dipivoxil for HBeAg-negative chronic hepatitis B for up to 5 years. Gastroenterology 2006;131:1743-1751.

326. Villeneuve JP, Durantel D, Durantel S, Westland C, Xiong S, Brosgart $C L$, et al. Selection of a hepatitis $B$ virus strain resistant to adefovir in a liver transplantation patient. J Hepatol 2003;39:10851089.

327. Villet S, Pichoud C, Billioud G, Barraud L, Durantel S, Trépo C, et al. Impact of hepatitis B virus rtA181V/T mutants on hepatitis B treatment failure. J Hepatol 2008;48:747-755.

328. Lampertico P, Viganò M, Manenti E, lavarone M, Sablon E, Colombo M. Low resistance to adefovir combined with lamivudine: a 3-year study of 145 lamivudine-resistant hepatitis B patients. Gastroenterology 2007;133:1445-1451.

329. Delaney WE 4th, Ray AS, Yang H, Qi X, Xiong S, Zhu Y, et al. Intracellular metabolism and in vitro activity of tenofovir against hepatitis B virus. Antimicrob Agents Chemother 2006;50:2471-2477.

330. Sheldon J, Camino N, Rodés B, Bartholomeusz A, Kuiper M, Tacke $F$, et al. Selection of hepatitis $B$ virus polymerase mutations in HIV-coinfected patients treated with tenofovir. Antivir Ther 2005; 10:727-734 
331. Amini-Bavil-Olyaee S, Herbers U, Sheldon J, Luedde T, Trautwein C, Tacke F. The rtA194T polymerase mutation impacts viral replication and susceptibility to tenofovir in hepatitis B e antigen-positive and hepatitis $B$ e antigen-negative hepatitis B virus strains. Hepatology 2009:49:1158-1165.

332. Shirvani-Dastgerdi E, Winer BY, Celià-Terrassa T, Kang Y, Tabernero $D$, Yagmur $E$, et al. Selection of the highly replicative and partially multidrug resistant rtS78T HBV polymerase mutation during TDFETV combination therapy. J Hepatol 2017;67:246-254.

333. Park ES, Lee AR, Kim DH, Lee JH, Yoo JJ, Ahn SH, et al. Identification of a quadruple mutation that confers tenofovir resistance in chronic hepatitis B patients. J Hepatol 2019;70:1093-1102.

334. Ahn SH, Kim W, Jung YK, Yang JM, Jang JY, Kweon YO, et al. Efficacy and safety of besifovir dipivoxil maleate compared with tenofovir disoproxil fumarate in treatment of chronic hepatitis B virus infection. Clin Gastroenterol Hepatol. 2018 Nov 15. pii: S15423565(18)31244-8. doi: 10.1016/j.cgh.2018.11.001.

335. Yuen MF, Ahn SH, Lee KS, Um SH, Cho M, Yoon SK, et al. Two-year treatment outcome of chronic hepatitis $B$ infection treated with besifovir vs. entecavir: results from a multicentre study. J Hepatol 2015;62:526-532.

336. Yim HJ, Hussain M, Liu Y, Wong SN, Fung SK, Lok AS. Evolution of multi-drug resistant hepatitis B virus during sequential therapy. Hepatology 2006;44:703-712.

337. Lee YS, Suh DJ, Lim YS, Jung SW, Kim KM, Lee HC, et al. Increased risk of adefovir resistance in patients with lamivudine-resistant chronic hepatitis $B$ after 48 weeks of adefovir dipivoxil monotherapy. Hepatology 2006;43:1385-1391.

338. Yeon JE, Yoo W, Hong SP, Chang YJ, Yu SK, Kim JH, et al. Resistance to adefovir dipivoxil in lamivudine resistant chronic hepatitis B patients treated with adefovir dipivoxil. Gut 2006;55:1488-1495.

339. Yim HJ. Management of antiviral-resistant chronic hepatitis B virus infection. Korean J Gastroenterol 2008;51:346-359.

340. Fung S, Kwan P, Fabri M, Horban A, Pelemis M, Hann HW, et al. Randomized comparison of tenofovir disoproxil fumarate vs emtricitabine and tenofovir disoproxil fumarate in patients with lamivudine-resistant chronic hepatitis B. Gastroenterology 2014;146:980988.

341. Lim YS, Byun KS, Yoo BC, Kwon SY, Kim YJ, An J, et al. Tenofovir monotherapy versus tenofovir and entecavir combination therapy in patients with entecavir-resistant chronic hepatitis B with multiple drug failure: results of a randomised trial. Gut 2016;65:852-860.

342. Lee S, Ahn SH, Jung KS, Kim DY, Kim BK, Kim SU, et al. Tenofovir versus tenofovir plus entecavir for chronic hepatitis $B$ with lamivudine resistance and entecavir resistance. J Viral Hepat 2017;24:141147.

343. Berg T, Zoulim F, Moeller B, Trinh H, Marcellin P, Chan S, et al. Long-term efficacy and safety of emtricitabine plus tenofovir DF vs. tenofovir DF monotherapy in adefovir-experienced chronic hepatitis B patients. J Hepatol 2014;60:715-722.

344. Lim YS, Yoo BC, Byun KS, Kwon SY, Kim YJ, An J, et al. Tenofovir monotherapy versus tenofovir and entecavir combination therapy in adefovir-resistant chronic hepatitis B patients with multiple drug failure: results of a randomised trial. Gut 2016;65:1042-1051.

345. Lim YS, Lee YS, Gwak GY, Byun KS, Kim YJ, Choi J, et al. Monotherapy with tenofovir disoproxil fumarate for multiple drug-resistant chronic hepatitis B: 3-year trial. Hepatology 2017;66:772-783.

346. Chung GE, Cho EJ, Lee JH, Yoo JJ, Lee M, Cho Y, et al. Tenofovir has inferior efficacy in adefovir-experienced chronic hepatitis $B$ patients compared to nucleos(t)ide-naïve patients. Clin Mol Hepatol 2017;23:66-73.

347. Yim HJ, Hwang SG. Options for the management of antiviral resistance during hepatitis B therapy: reflections on battles over a decade. Clin Mol Hepatol 2013;19:195-209.

348. Park JY, Kim CW, Bae SH, Jung KS, Kim HY, Yoon SK, et al. Entecavir plus tenofovir combination therapy in patients with multidrugresistant chronic hepatitis $B$ : results of a multicentre, prospective study. Liver Int 2016;36:1108-1115.

349. Yim HJ, Suh SJ, Jung YK, Hwang SG, Park H, Seo YS, et al. Tenofovir-based combination therapy or monotherapy for multi-drug resistant chronic hepatitis $B$; real world data from multicenter cohort study [Abstract]. Hepatology 2017;66:12A.

350. Lee JH, Lee YB, Cho H, Cho YY, Cho EJ, Kim YJ, et al. Identification of a triple mutation that confers tenofovir resistance in chronic hepatitis B patients [Abstract]. Hepatology 2017;66:69A-70A.

351. Heo J, Park JY, Lee HJ, Tak WY, Um SH, Kim DY, et al. A 96-week randomized trial of switching to entecavir in chronic hepatitis $\mathrm{B}$ patients with a partial virological response to lamivudine. Antivir Ther 2012;17:1563-1570.

352. Yim HJ, Kim IH, Suh SJ, Jung YK, Kim JH, Seo YS, et al. Switching to tenofovir vs continuing entecavir for hepatitis B virus with partial virologic response to entecavir: a randomized controlled trial. J Viral Hepat 2018;25:1321-1330.

353. Chen J, Zhao SS, Liu XX, Huang ZB, Huang Y. Comparison of the efficacy of tenofovir versus tenofovir plus entecavir in the treatment of chronic hepatitis B in patients with poor efficacy of entecavir: a systematic review and meta-analysis. Clin Ther 2017;39:18701880.

354. Jeong DI, Jung SW, Park BR, Lee BU, Park JH, Kim BG, et al. Clinical course of partial virologic response with prolonged tenofovir therapy in nuclos(t)ides-naive patients with chronic hepatitis B. Dig Dis Sci 2017;62:2908-2914.

355. Lee HW, Park JY, Lee JW, Yoon KT, Kim CW, Park H, et al. Longterm efficacy of tenofovir disoproxil fumarate monotherapy for multidrug-resistant chronic HBV infection. Clin Gastroenterol Hepatol. 2018 Oct 26. pii: S1542-3565(18)31201-1. doi: 10.1016/ 
j.cgh.2018.10.037.

356. Kim DY, Lee HW, Song JE, Kim BK, Kim SU, Kim DY, et al. Switching from tenofovir and nucleoside analogue therapy to tenofovir monotherapy in virologically suppressed chronic hepatitis B patients with antiviral resistance. J Med Virol 2018;90:497-502.

357. Nagata N, Kagawa T, Hirose S, Arase Y, Tsuruya K, Anzai K, et al. Off-treatment durability of antiviral response to nucleoside analogues in patients with chronic hepatitis B. BMC Gastroenterol 2016;16:38.

358. Ning Q, Han M, Sun Y, Jiang J, Tan D, Hou J, et al. Switching from entecavir to PegIFN alfa-2a in patients with $\mathrm{HBeAg}$-positive chronic hepatitis B: a randomised open-label trial (OSST trial). J Hepatol 2014;61:777-784.

359. Hu P, Shang J, Zhang W, Gong G, Li Y, Chen X, et al. HBsAg loss with Peg-interferon Alfa-2a in hepatitis B patients with partial response to nucleos(t)ide analog: new switch study. J Clin Transl Hepatol 2018;6:25-34.

360. Xie Q, Zhou H, Bai X, Wu S, Chen JJ, Sheng J, et al. A randomized, open-label clinical study of combined pegylated interferon Alfa-2a (40KD) and entecavir treatment for hepatitis B " $e$ " antigen-positive chronic hepatitis B. Clin Infect Dis 2014;59:1714-1723.

361. Li GJ, Yu YQ, Chen SL, Fan P, Shao LY, Chen JZ, et al. Sequential combination therapy with pegylated interferon leads to loss of hepatitis B surface antigen and hepatitis B e antigen ( $\mathrm{HBeAg}$ ) seroconversion in $\mathrm{HBeAg}$-positive chronic hepatitis $\mathrm{B}$ patients receiving long-term entecavir treatment. Antimicrob Agents Chemother 2015;59:4121-4128.

362. Zhang W, Xie Q, Ning Q, Dou X, Chen X, Jia J, et al. The role of peginterferon in nucleos(t)ide-analogue-treated chronic hepatitis B patients: a review of published literature. J Viral Hepat 2017;24:618-623.

363. Bourlière $M$, Rabiega $P$, Ganne-Carrie N, Serfaty L, Marcellin $P$, Barthe $Y$, et al. Effect on HBs antigen clearance of addition of pegylated interferon alfa-2a to nucleos(t)ide analogue therapy versus nucleos(t)ide analogue therapy alone in patients with HBe antigennegative chronic hepatitis $B$ and sustained undetectable plasma hepatitis B virus DNA: a randomised, controlled, open-label trial. Lancet Gastroenterol Hepatol 2017;2:177-188.

364. Lee JH, Chung S, Kim MS, Kim SW, Yoon JS, Chang Y, et al. Entecavir plus pegylated interferon alfa-2a and sequential HBV vaccination increases the chance of $\mathrm{HBsAg}$-seroclearance: a results from randomized controlled E+VIP Trial [Abstract]. Clin Mol Hepatol 2018;24(Suppl 3):2.

365. Rijckborst V, Hansen BE, Ferenci P, Brunetto MR, Tabak F, Cakaloglu $Y$, et al. Validation of a stopping rule at week 12 using $\mathrm{HBsAg}$ and HBV DNA for HBeAg-negative patients treated with peginterferon alfa-2a. J Hepatol 2012;56:1006-1011.

366. Wu CY, Chen YJ, Ho HJ, Hsu YC, Kuo KN, Wu MS, et al. Association between nucleoside analogues and risk of hepatitis B virus-related hepatocellular carcinoma recurrence following liver resection. JAMA 2012;308:1906-1914.

367. Huang G, Li PP, Lau WY, Pan ZY, Zhao LH, Wang ZG, et al. Antiviral therapy reduces hepatocellular carcinoma recurrence in patients with low HBV-DNA levels: a randomized controlled trial. Ann Surg 2018;268:943-954.

368. Wong JS, Wong GL, Tsoi KK, Wong VW, Cheung SY, Chong CN, et al. Meta-analysis: the efficacy of anti-viral therapy in prevention of recurrence after curative treatment of chronic hepatitis B-related hepatocellular carcinoma. Aliment Pharmacol Ther 2011;33:11041112.

369. Cho H, Ahn H, Lee DH, Lee JH, Jung YJ, Chang Y, et al. Entecavir and tenofovir reduce hepatitis $B$ virus-related hepatocellular carcinoma recurrence more effectively than other antivirals. J Viral Hepat 2018;25:707-717.

370. Chen LT, Chen MF, Li LA, Lee PH, Jeng LB, Lin DY, et al. Long-term results of a randomized, observation-controlled, phase III trial of adjuvant interferon Alfa-2b in hepatocellular carcinoma after curative resection. Ann Surg 2012;255:8-17.

371. Kubo S, Nishiguchi S, Hamba H, Hirohashi K, Tanaka H, Shuto T, et al. Reactivation of viral replication after liver resection in patients infected with hepatitis B virus. Ann Surg 2001;233:139-145.

372. Huang L, Li J, Yan J, Sun J, Zhang X, Wu M, et al. Antiviral therapy decreases viral reactivation in patients with hepatitis $B$ virus-related hepatocellular carcinoma undergoing hepatectomy: a randomized controlled trial. J Viral Hepat 2013;20:336-342.

373. Dan JQ, Zhang YJ, Huang JT, Chen MS, Gao HJ, Peng ZW, et al. Hepatitis $B$ virus reactivation after radiofrequency ablation or hepatic resection for $\mathrm{HBV}$-related small hepatocellular carcinoma: a retrospective study. Eur J Surg Oncol 2013;39:865-872.

374. Yoshida H, Yoshida H, Goto E, Sato T, Ohki T, Masuzaki R, et al. Safety and efficacy of lamivudine after radiofrequency ablation in patients with hepatitis B virus-related hepatocellular carcinoma. Hepatol Int 2008;2:89-94.

375. Lao XM, Luo G, Ye LT, Luo C, Shi M, Wang D, et al. Effects of antiviral therapy on hepatitis $B$ virus reactivation and liver function after resection or chemoembolization for hepatocellular carcinoma. Liver Int 2013;33:595-604.

376. Lao XM, Wang D, Shi M, Liu G, Li S, Guo R, et al. Changes in hepatitis $B$ virus DNA levels and liver function after transcatheter arterial chemoembolization of hepatocellular carcinoma. Hepatol Res 2011:41:553-563.

377. Firpi RJ, Nelson DR. Management of viral hepatitis in hematologic malignancies. Blood Rev 2008;22:117-126.

378. Jang JW, Choi JY, Bae SH, Yoon SK, Chang UI, Kim CW, et al. A randomized controlled study of preemptive lamivudine in patients receiving transarterial chemo-lipiodolization. Hepatology 
2006:43:233-240.

379. Park JW, Park KW, Cho SH, Park HS, Lee WJ, Lee DH, et al. Risk of hepatitis $\mathrm{B}$ exacerbation is low after transcatheter arterial chemoembolization therapy for patients with HBV-related hepatocelIular carcinoma: report of a prospective study. Am J Gastroenterol 2005;100:2194-2200.

380. Yeo W, Lam KC, Zee B, Chan PS, Mo FK, Ho WM, et al. Hepatitis B reactivation in patients with hepatocellular carcinoma undergoing systemic chemotherapy. Ann Oncol 2004;15:1661-1666.

381. Nagamatsu H, Itano S, Nagaoka S, Akiyoshi J, Matsugaki S, Kurogi J, et al. Prophylactic lamivudine administration prevents exacerbation of liver damage in $\mathrm{HBe}$ antigen positive patients with hepatocellular carcinoma undergoing transhepatic arterial infusion chemotherapy. Am J Gastroenterol 2004;99:2369-2375.

382. Tamori A, Nishiguchi S, Tanaka M, Kurooka H, Fujimoto S, Nakamura $\mathrm{K}$, et al. Lamivudine therapy for hepatitis $B$ virus reactivation in a patient receiving intra-arterial chemotherapy for advanced hepatocellular carcinoma. Hepatol Res 2003;26:77-80.

383. Kim JH, Park JW, Kim TH, Koh DW, Lee WJ, Kim CM. Hepatitis B virus reactivation after three-dimensional conformal radiotherapy in patients with hepatitis B virus-related hepatocellular carcinoma. Int J Radiat Oncol Biol Phys 2007;69:813-819.

384. Jang JW, Kwon JH, You CR, Kim JD, Woo HY, Bae SH, et al. Risk of $\mathrm{HBV}$ reactivation according to viral status and treatment intensity in patients with hepatocellular carcinoma. Antivir Ther 2011;16:969-977.

385. Shim JH, Park JW, Choi JI, Park BJ, Kim CM. Practical efficacy of sorafenib monotherapy for advanced hepatocellular carcinoma patients in a Hepatitis B virus-endemic area. J Cancer Res Clin Oncol 2009;135:617-625.

386. Suh SJ, Yim HJ, Seo JH, Lee YS, Hyun JJ, Jung YK, et al. The risk of hepatitis $\mathrm{B}$ virus reactivation is considerably high during sorafenib therapy in patients with advanced hepatocellular carcinoma. J Hepatol 2017;66:S449-S450.

387. Loomba R, Liang TJ. Hepatitis B reactivation associated with immune suppressive and biological modifier therapies: current concepts, management strategies, and future directions. Gastroenterology 2017;152:1297-1309.

388. Lampertico $\mathrm{P}$, Chan $\mathrm{HL}$, Janssen $\mathrm{HL}$, Strasser SI, Schindler R, Berg T. Review article: long-term safety of nucleoside and nucleotide analogues in HBV-monoinfected patients. Aliment Pharmacol Ther 2016;44:16-34.

389. Gracey DM, Snelling P, McKenzie P, Strasser SI. Tenofovir-associated Fanconi syndrome in patients with chronic hepatitis B monoinfection. Antivir Ther 2013;18:945-948.

390. Maggi P, Montinaro V, Leone A, Fasano M, Volpe A, Bellacosa C, et al. Bone and kidney toxicity induced by nucleotide analogues in patients affected by HBV-related chronic hepatitis: a longitudinal study. J Antimicrob Chemother 2015;70:1150-1154.

391. López-Alcorocho JM, Barril G, Ortiz-Movilla N, Traver JA, Bartolomé J, Sanz P, et al. Prevalence of hepatitis B, hepatitis C, GB virus $C$ /hepatitis $G$ and TT viruses in predialysis and hemodialysis patients. J Med Virol 2001;63:103-107.

392. Gwak GY, Huh W, Lee DH, Min BH, Koh KC, Kim JJ, et al. Occult hepatitis $B$ virus infection in chronic hemodialysis patients in Korea. Hepatogastroenterology 2008;55:1721-1724.

393. Minuk GY, Sun DF, Greenberg R, Zhang M, Hawkins K, Uhanova J, et al. Occult hepatitis $B$ virus infection in a North American adult hemodialysis patient population. Hepatology 2004;40:1072-1077.

394. Burdick RA, Bragg-Gresham JL, Woods JD, Hedderwick SA, Kurokawa $\mathrm{K}$, Combe $\mathrm{C}$, et al. Patterns of hepatitis B prevalence and seroconversion in hemodialysis units from three continents: the DOPPS. Kidney Int 2003;63:2222-2229.

395. Johnson DW, Dent H, Yao Q, Tranaeus A, Huang CC, Han DS, et al. Frequencies of hepatitis $B$ and $C$ infections among haemodialysis and peritoneal dialysis patients in Asia-Pacific countries: analysis of registry data. Nephrol Dial Transplant 2009;24:1598-1603.

396. Finelli L, Miller JT, Tokars JI, Alter MJ, Arduino MJ. National surveillance of dialysis-associated diseases in the United States, 2002. Semin Dial 2005;18:52-61.

397. Hu TH, Huang PY, Chang KC, Tseng PL, Yen YH, Tsai MC. Chronic hepatitis $B$ patient treated with Tenofovir (TDF) may experience increased risks of orthopedic disorders and renal deficits compared to those treated with Enetecavir (ETV) [Abstract]. Hepatology 2018;68(S1):264A.

398. Lai CL, Ahn SH, Lee KS, Um SH, Cho M, Yoon SK, et al. Phase IIb multicentred randomised trial of besifovir (LB80380) versus entecavir in Asian patients with chronic hepatitis B. Gut 2014;63:9961004.

399. Gane E, Seto WK, Janssen H, Caruntu FA, Kim HJ, Abdurakhmanov $D$, et al. Safety and efficacy at 1 year after switching from Tenofovir Disoproxil Fumurate to Tenofovir Alafenamide in chronic HBV patients with risk factors for TDF use [Abstract]. J Hepatol 2018;68(S1):S87.

400. Yuki N, Nagaoka T, Yamashiro M, Mochizuki K, Kaneko A, Yamamoto $K$, et al. Long-term histologic and virologic outcomes of acute self-limited hepatitis B. Hepatology 2003;37:1172-1179.

401. Shiffman ML. Management of acute hepatitis B. Clin Liver Dis 2010;14:75-91; viii-ix.

402. Lampertico P, Maini M, Papatheodoridis G. Optimal management of hepatitis B virus infection - EASL Special Conference. J Hepatol 2015;63:1238-1253.

403. European Association for the Study of the Liver. EASL Clinical Practical Guidelines on the management of acute (fulminant) liver failure. J Hepatol 2017;66:1047-1081.

404. Böcher WO, Herzog-Hauff S, Herr W, Heermann K, Gerken G, Mey- 
er Zum Büschenfelde KH, et al. Regulation of the neutralizing antihepatitis $B$ surface (HBs) antibody response in vitro in $H B s$ vaccine recipients and patients with acute or chronic hepatitis B virus (HBV) infection. Clin Exp Immunol 1996;105:52-58.

405. Mantzoukis K, Rodríguez-Perálvarez M, Buzzetti E, Thorburn D, Davidson BR, Tsochatzis $E$, et al. Pharmacological interventions for acute hepatitis B infection: an attempted network meta-analysis. Cochrane Database Syst Rev 2017;3:CD011645.

406. Kumar M, Satapathy S, Monga R, Das K, Hissar S, Pande C, et al. A randomized controlled trial of lamivudine to treat acute hepatitis $B$. Hepatology 2007:45:97-101.

407. Yu JW, Sun LJ, Zhao YH, Kang P, Li SC. The study of efficacy of lamivudine in patients with severe acute hepatitis B. Dig Dis Sci 2010:55:775-783.

408. Jochum C, Maischack F, Anastasiou OE, Verheyen J, Timm J, Bechmann $L$, et al. Treatment of fulminant acute Hepatitis B with nucles(t)id analogues is safe and does not lead to secondary chronification of Hepatitis B. Z Gastroenterol 2016;54:1306-1311.

409. Tillmann HL, Hadem J, Leifeld L, Zachou K, Canbay A, Eisenbach $C$, et al. Safety and efficacy of lamivudine in patients with severe acute or fulminant hepatitis $B$, a multicenter experience. J Viral Hepat 2006;13:256-263.

410. Miyake Y, Iwasaki Y, Takaki A, Fujioka S, Takaguchi K, Ikeda H, et al. Lamivudine treatment improves the prognosis of fulminant hepatitis B. Intern Med 2008;47:1293-1299.

411. Streinu-Cercel A, Sandulescu O, Stefan M, Streinu-Cercel A. Treatment with lamivudine and entecavir in severe acute hepatitis $B$. Indian J Med Microbiol 2016;34:166-172.

412. Gerada J, Borg E, Formosa D, Magro R, Pocock J. Tenofovir as rescue therapy following clinical failure to Lamivudine in severe acute hepatitis B. Mediterr J Hematol Infect Dis 2013;5:e2013035.

413. Gupta S, Govindarajan S, Fong TL, Redeker AG. Spontaneous reactivation in chronic hepatitis B: patterns and natural history. J Clin Gastroenterol 1990;12:562-568.

414. Lok AS, McMahon BJ; Practice Guidelines Committee, American Association for the Study of Liver Diseases (AASLD). Chronic hepatitis B: update of recommendations. Hepatology 2004;39:857-861.

415. Tanaka Y, Esumi M, Shikata T. Persistence of hepatitis B virus DNA after serological clearance of hepatitis B virus. Liver 1990;10:6-10.

416. Koo YX, Tan DS, Tan IB, Tao M, Chow WC, Lim ST. Hepatitis B virus reactivation and role of antiviral prophylaxis in lymphoma patients with past hepatitis $B$ virus infection who are receiving chemoimmunotherapy. Cancer 2010;116:115-121.

417. Koo YX, Tay M, Teh YE, Teng D, Tan DS, Tan IB, et al. Risk of hepatitis $B$ virus (HBV) reactivation in hepatitis $B$ surface antigen negative/hepatitis $B$ core antibody positive patients receiving rituximabcontaining combination chemotherapy without routine antiviral prophylaxis. Ann Hematol 2011;90:1219-1223.
418. Yeo W, Johnson PJ. Diagnosis, prevention and management of hepatitis $B$ virus reactivation during anticancer therapy. Hepatology 2006;43:209-220

419. Hwang JP, Lok AS. Management of patients with hepatitis B who require immunosuppressive therapy. Nat Rev Gastroenterol Hepatol 2014;11:209-219.

420. Bessone $F$, Dirchwolf M. Management of hepatitis B reactivation in immunosuppressed patients: an update on current recommendations. World J Hepatol 2016;8:385-394.

421. Lok AS, Liang RH, Chiu EK, Wong KL, Chan TK, Todd D. Reactivation of hepatitis $B$ virus replication in patients receiving cytotoxic therapy. Report of a prospective study. Gastroenterology 1991;100:182-188.

422. Yeo W, Chan PK, Zhong S, Ho WM, Steinberg JL, Tam JS, et al. Frequency of hepatitis $B$ virus reactivation in cancer patients undergoing cytotoxic chemotherapy: a prospective study of 626 patients with identification of risk factors. J Med Virol 2000;62:299-307.

423. Loomba R, Rowley A, Wesley R, Liang TJ, Hoofnagle JH, Pucino $F$, et al. Systematic review: the effect of preventive lamivudine on hepatitis $B$ reactivation during chemotherapy. Ann Intern Med 2008;148:519-528.

424. Kwak LW, Halpern J, Olshen RA, Horning SJ. Prognostic significance of actual dose intensity in diffuse large-cell lymphoma: results of a tree-structured survival analysis. J Clin Oncol 1990;8:963-977.

425. Bonadonna G, Valagussa P, Moliterni A, Zambetti M, Brambilla C. Adjuvant cyclophosphamide, methotrexate, and fluorouracil in node-positive breast cancer: the results of 20 years of follow-up. N Engl J Med 1995;332:901-906.

426. Yeo W, Chan PK, Hui P, Ho WM, Lam KC, Kwan WH, et al. Hepatitis $B$ virus reactivation in breast cancer patients receiving cytotoxic chemotherapy: a prospective study. J Med Virol 2003;70:553-561.

427. Jang JW. Hepatitis B virus reactivation in patients with hepatocellular carcinoma undergoing anti-cancer therapy. World J Gastroenterol 2014;20:7675-7685.

428. Perrillo RP, Gish R, Falck-Ytter YT. American Gastroenterological Association Institute technical review on prevention and treatment of hepatitis B virus reactivation during immunosuppressive drug therapy. Gastroenterology 2015;148:221-244.e3.

429. Reddy KR, Beavers KL, Hammond SP, Lim JK, Falck-Ytter YT; American Gastroenterological Association Institute. American Gastroenterological Association Institute guideline on the prevention and treatment of hepatitis B virus reactivation during immunosuppressive drug therapy. Gastroenterology 2015;148:215-219; quiz e16e17.

430. Takai S, Tsurumi H, Ando K, Kasahara S, Sawada M, Yamada T, et al. Prevalence of hepatitis $B$ and $C$ virus infection in haematological malignancies and liver injury following chemotherapy. Eur J Haematol 2005;74:158-165. 
431. Hsu C, Hsiung CA, Su IJ, Hwang WS, Wang MC, Lin SF, et al. A revisit of prophylactic lamivudine for chemotherapy-associated hepatitis $B$ reactivation in non-Hodgkin's lymphoma: a randomized trial. Hepatology 2008;47:844-853.

432. Engels EA, Cho ER, Jee SH. Hepatitis B virus infection and risk of non-Hodgkin lymphoma in South Korea: a cohort study. Lancet Oncol 2010;11:827-834.

433. Cheng AL, Hsiung CA, Su IJ, Chen PJ, Chang MC, Tsao CJ, et al. Steroid-free chemotherapy decreases risk of hepatitis B virus (HBV) reactivation in HBV-carriers with lymphoma. Hepatology 2003;37:1320-1328.

434. Yeo W, Chan TC, Leung NW, Lam WY, Mo FK, Chu MT, et al. Hepatitis $B$ virus reactivation in lymphoma patients with prior resolved hepatitis B undergoing anticancer therapy with or without rituximab. J Clin Oncol 2009;27:605-611.

435. Dong HJ, Ni LN, Sheng GF, Song HL, Xu JZ, Ling Y. Risk of hepatitis $B$ virus (HBV) reactivation in non-Hodgkin lymphoma patients receiving rituximab-chemotherapy: a meta-analysis. J Clin Virol 2013;57:209-214.

436. Chen XQ, Peng JW, Lin GN, Li M, Xia ZJ. The effect of prophylactic lamivudine on hepatitis $B$ virus reactivation in $\mathrm{HBsAg-positive}$ patients with diffuse large B-cell lymphoma undergoing prolonged rituximab therapy. Med Oncol 2012;29:1237-1241.

437. Zurawska U, Hicks LK, Woo G, Bell CM, Krahn M, Chan KK, et al. Hepatitis B virus screening before chemotherapy for lymphoma: a cost-effectiveness analysis. J Clin Oncol 2012;30:3167-3173.

438. Buti M, Manzano ML, Morillas RM, García-Retortillo M, Martín L, Prieto $M$, et al. Randomized prospective study evaluating tenofovir disoproxil fumarate prophylaxis against hepatitis B virus reactivation in anti-HBc-positive patients with rituximab-based regimens to treat hematologic malignancies: the Preblin study. PLoS One 2017;12:e0184550

439. Lau GK, He ML, Fong DY, Bartholomeusz A, Au WY, Lie AK, et al. Preemptive use of lamivudine reduces hepatitis $B$ exacerbation after allogeneic hematopoietic cell transplantation. Hepatology 2002;36:702-709.

440. Sarmati L, Andreoni M, Antonelli G, Arcese W, Bruno R, Coppola $N$, et al. Recommendations for screening, monitoring, prevention, prophylaxis and therapy of hepatitis B virus reactivation in patients with haematologic malignancies and patients who underwent haematologic stem cell transplantation-a position paper. Clin Microbiol Infect 2017;23:935-940.

441. Chen CY, Tien FM, Cheng A, Huang SY, Chou WC, Yao M, et al. Hepatitis B reactivation among 1962 patients with hematological malignancy in Taiwan. BMC Gastroenterol 2018;18:6.

442. Chen FW, Coyle L, Jones BE, Pattullo V. Entecavir versus lamivudine for hepatitis B prophylaxis in patients with haematological disease. Liver Int 2013;33:1203-1210.
443. Kim MK, Ahn JH, Kim SB, Im YS, Lee SI, Ahn SH, et al. Hepatitis B reactivation during adjuvant anthracycline-based chemotherapy in patients with breast cancer: a single institution's experience. Korean J Intern Med 2007;22:237-243.

444. Dai MS, Wu PF, Shyu RY, Lu JJ, Chao TY. Hepatitis B virus reactivation in breast cancer patients undergoing cytotoxic chemotherapy and the role of preemptive lamivudine administration. Liver Int 2004; $24: 540-546$.

445. Wu YT, Li X, Liu ZL, Xu Z, Dai W, Zhang K, et al. Hepatitis B virus reactivation and antiviral prophylaxis during lung cancer chemotherapy: a systematic review and meta-analysis. PLoS One 2017;12:e0179680.

446. Xu Z, Dai W, Wu YT, Arshad B, Li X, Wu H, et al. Prophylactic effect of lamivudine on chemotherapy-induced hepatitis B virus reactivation in patients with solid tumour: a meta-analysis. Eur J Cancer Care (Engl) 2018;27:e12799.

447. El-Sayed MH, Mohamed MM, Karim A, Maina AM, Oliveri F, Brunetto MR, et al. Severe liver disease is caused by HBV rather than $\mathrm{HCV}$ in children with hematological malignancies. Hematol J 2003;4:321-327.

448. Yeo W, Chan PK, Ho WM, Zee B, Lam KC, Lei KI, et al. Lamivudine for the prevention of hepatitis $B$ virus reactivation in hepatitis $B$ santigen seropositive cancer patients undergoing cytotoxic chemotherapy. J Clin Oncol 2004;22:927-934.

449. Chung SJ, Kim JK, Park MC, Park YB, Lee SK. Reactivation of hepatitis $B$ viral infection in inactive $\mathrm{HBsAg}$ carriers following anti-tumor necrosis factor-alpha therapy. J Rheumatol 2009;36:2416-2420.

450. Esteve M, Saro C, González-Huix F, Suarez F, Forné M, Viver JM. Chronic hepatitis $B$ reactivation following infliximab therapy in Crohn's disease patients: need for primary prophylaxis. Gut 2004:53:1363-1365.

451. Kim YJ, Bae SC, Sung YK, Kim TH, Jun JB, Yoo DH, et al. Possible reactivation of potential hepatitis $B$ virus occult infection by tumor necrosis factor-alpha blocker in the treatment of rheumatic diseases. J Rheumatol 2010;37:346-350.

452. Lee $\mathrm{YH}$, Bae SC, Song GG. Hepatitis B virus reactivation in HBsAgpositive patients with rheumatic diseases undergoing anti-tumor necrosis factor therapy or DMARDs. Int J Rheum Dis 2013;16:527531.

453. Pérez-Alvarez R, Díaz-Lagares C, García-Hernández F, Lopez-Roses L, Brito-Zerón P, Pérez-de-Lis $M$, et al. Hepatitis B virus (HBV) reactivation in patients receiving tumor necrosis factor (TNF)-targeted therapy: analysis of 257 cases. Medicine (Baltimore) 2011;90:359371.

454. Lau GK, Yiu HH, Fong DY, Cheng HC, Au WY, Lai LS, et al. Early is superior to deferred preemptive lamivudine therapy for hepatitis $B$ patients undergoing chemotherapy. Gastroenterology 2003;125:1742-1749 
455. Saab S, Dong MH, Joseph TA, Tong MJ. Hepatitis B prophylaxis in patients undergoing chemotherapy for lymphoma: a decision analysis model. Hepatology 2007;46:1049-1056.

456. Viganò M, Serra G, Casella G, Grossi G, Lampertico P. Reactivation of hepatitis $B$ virus during targeted therapies for cancer and immune-mediated disorders. Expert Opin Biol Ther 2016;16:917926.

457. Hui CK, Cheung WW, Au WY, Lie AK, Zhang HY, Yueng YH, et al. Hepatitis $B$ reactivation after withdrawal of pre-emptive lamivudine in patients with haematological malignancy on completion of cytotoxic chemotherapy. Gut 2005;54:1597-1603.

458. Cerva C, Colagrossi L, Maffongelli G, Salpini R, Di Carlo D, Malagnino $V$, et al. Persistent risk of HBV reactivation despite extensive lamivudine prophylaxis in haematopoietic stem cell transplant recipients who are anti-HBc-positive or HBV-negative recipients with an anti-HBc-positive donor. Clin Microbiol Infect 2016;22:946.e1-946.e8.

459. Liu WP, Wang XP, Zheng W, Ping LY, Zhang C, Wang GQ, et al. Hepatitis $B$ virus reactivation after withdrawal of prophylactic antiviral therapy in patients with diffuse large B cell lymphoma. Leuk Lymphoma 2016;57:1355-1362.

460. Nakaya A, Fujita S, Satake A, Nakanishi T, Azuma Y, Tsubokura Y, et al. Delayed HBV reactivation in rituximab-containing chemotherapy: how long should we continue anti-virus prophylaxis or monitoring HBV-DNA? Leuk Res 2016;50:46-49.

461. Paul S, Dickstein A, Saxena A, Terrin N, Viveiros K, Balk EM, et al. Role of surface antibody in hepatitis $B$ reactivation in patients with resolved infection and hematologic malignancy: a meta-analysis. Hepatology 2017;66:379-388.

462. Rossi G, Pelizzari A, Motta M, Puoti M. Primary prophylaxis with lamivudine of hepatitis $B$ virus reactivation in chronic $\mathrm{HbsAg}$ carriers with lymphoid malignancies treated with chemotherapy. $\mathrm{Br}$ J Haematol 2001;115:58-62.

463. Li HR, Huang JJ, Guo HQ, Zhang X, Xie Y, Zhu HL, et al. Comparison of entecavir and lamivudine in preventing hepatitis $B$ reactivation in lymphoma patients during chemotherapy. J Viral Hepat 2011;18:877-883.

464. Yu S, Luo H, Pan M, Luis AP, Xiong Z, Shuai P, et al. Comparison of entecavir and lamivudine in preventing HBV reactivation in lymphoma patients undergoing chemotherapy: a meta-analysis. Int J Clin Pharm 2016;38:1035-1043.

465. Demetris AJ, Jaffe R, Sheahan DG, Burnham J, Spero J, Iwatsuki $S$, et al. Recurrent hepatitis $B$ in liver allograft recipients. Differentiation between viral hepatitis B and rejection. Am J Pathol 1986:125:161-172.

466. Demetris AJ, Todo S, Van Thiel DH, Fung JJ, Iwaki Y, Sysyn G, et al. Evolution of hepatitis B virus liver disease after hepatic replacement. Practical and theoretical considerations. Am J Pathol
1990;137:667-676.

467. Freeman RB, Sanchez $H$, Lewis WD, Sherburne B, Dzik WH, Khettry $\mathrm{U}$, et al. Serologic and DNA follow-up data from HBsAg-positive patients treated with orthotopic liver transplantation. Transplantation 1991;51:793-797.

468. Lake JR, Wright TL. Liver transplantation for patients with hepatitis $B$ : what have we learned from our results? Hepatology 1991;13:796-799.

469. O'Grady JG, Smith HM, Davies SE, Daniels HM, Donaldson PT, Tan KC, et al. Hepatitis B virus reinfection after orthotopic liver transplantation. Serological and clinical implications. J Hepatol 1992;14:104-111.

470. Rizzetto M, Recchia S, Salizzoni M. Liver transplantation in carriers of the HBsAg. J Hepatol 1991;13:5-7.

471. Todo S, Demetris AJ, Van Thiel D, Teperman L, Fung JJ, Starzl TE. Orthotopic liver transplantation for patients with hepatitis $B$ virusrelated liver disease. Hepatology 1991;13:619-626.

472. Samuel D, Muller R, Alexander G, Fassati L, Ducot B, Benhamou JP, et al. Liver transplantation in European patients with the hepatitis B surface antigen. N Engl J Med 1993;329:1842-1847.

473. Han SH, Ofman J, Holt C, King K, Kunder G, Chen P, et al. An efficacy and cost-effectiveness analysis of combination hepatitis $B$ immune globulin and lamivudine to prevent recurrent hepatitis $B$ after orthotopic liver transplantation compared with hepatitis B immune globulin monotherapy. Liver Transpl 2000;6:741-748.

474. Markowitz JS, Martin P, Conrad AJ, Markmann JF, Seu P, Yersiz $\mathrm{H}$, et al. Prophylaxis against hepatitis $B$ recurrence following liver transplantation using combination lamivudine and hepatitis B immune globulin. Hepatology 1998;28:585-589.

475. Marzano A, Salizzoni M, Debernardi-Venon W, Smedile A, Franchello $A$, Ciancio $A$, et al. Prevention of hepatitis $B$ virus recurrence after liver transplantation in cirrhotic patients treated with lamivudine and passive immunoprophylaxis. J Hepatol 2001;34:903910.

476. Steinmüller T, Seehofer D, Rayes N, Müller AR, Settmacher U, Jonas $S$, et al. Increasing applicability of liver transplantation for patients with hepatitis B-related liver disease. Hepatology 2002;35:15281535.

477. Katz LH, Paul M, Guy DG, Tur-Kaspa R. Prevention of recurrent hepatitis $B$ virus infection after liver transplantation: hepatitis $B$ immunoglobulin, antiviral drugs, or both? Systematic review and meta-analysis. Transpl Infect Dis 2010;12:292-308.

478. Loomba R, Rowley AK, Wesley R, Smith KG, Liang TJ, Pucino F, et al. Hepatitis $B$ immunoglobulin and Lamivudine improve hepatitis B-related outcomes after liver transplantation: meta-analysis. Clin Gastroenterol Hepatol 2008;6:696-700.

479. Mutimer D, Dusheiko G, Barrett C, Grellier L, Ahmed M, Anschuetz $G$, et al. Lamivudine without HBIg for prevention of graft 
reinfection by hepatitis B: long-term follow-up. Transplantation 2000;70:809-815.

480. Perrillo RP, Wright T, Rakela J, Levy G, Schiff E, Gish R, et al. A multicenter United States-Canadian trial to assess lamivudine monotherapy before and after liver transplantation for chronic hepatitis $B$. Hepatology 2001;33:424-432.

481. Gane EJ, Patterson S, Strasser SI, McCaughan GW, Angus PW. Combination of lamivudine and adefovir without hepatitis B immune globulin is safe and effective prophylaxis against hepatitis $B$ virus recurrence in hepatitis $B$ surface antigen-positive liver transplant candidates. Liver Transpl 2013;19:268-274.

482. Fung J, Chan SC, Cheung C, Yuen MF, Chok KS, Sharr W, et al. Oral nucleoside/nucleotide analogs without hepatitis B immune globulin after liver transplantation for hepatitis B. Am J Gastroenterol 2013;108:942-948.

483. Fung J, Cheung C, Chan SC, Yuen MF, Chok KS, Sharr W, et al. Entecavir monotherapy is effective in suppressing hepatitis $B$ virus after liver transplantation. Gastroenterology 2011;141:1212-1219.

484. Fung J, Wong T, Chok K, Chan A, Cheung TT, Dai JW, et al. Longterm outcomes of entecavir monotherapy for chronic hepatitis $\mathrm{B}$ after liver transplantation: results up to 8 years. Hepatology 2017;66:1036-1044.

485. Cholongitas E, Papatheodoridis GV. High genetic barrier nucleos $(\mathrm{t})$ ide analogue(s) for prophylaxis from hepatitis $B$ virus recurrence after liver transplantation: a systematic review. Am J Transplant 2013;13:353-362.

486. Gane EJ, Angus PW, Strasser S, Crawford DH, Ring J, Jeffrey GP, et al. Lamivudine plus low-dose hepatitis B immunoglobulin to prevent recurrent hepatitis B following liver transplantation. Gastroenterology 2007;132:931-937.

487. Cholongitas E, Goulis I, Antoniadis N, Fouzas I, Imvrios G, Papanikolaou $\mathrm{V}$, et al. New nucleos(t)ide analogue monoprophylaxis after cessation of hepatitis $B$ immunoglobulin is effective against hepatitis B recurrence. Transpl Int 2014;27:1022-1028.

488. Cholongitas E, Vasiliadis T, Antoniadis N, Goulis I, Papanikolaou V, Akriviadis $\mathrm{E}$. Hepatitis B prophylaxis post liver transplantation with newer nucleos(t)ide analogues after hepatitis B immunoglobulin discontinuation. Transpl Infect Dis 2012;14:479-487.

489. European Association for the Study of the Liver. EASL Clinical Practice Guidelines: Liver transplantation. J Hepatol 2016;64:433-485.

490. Perrillo R, Buti M, Durand F, Charlton M, Gadano A, Cantisani G, et al. Entecavir and hepatitis $B$ immune globulin in patients undergoing liver transplantation for chronic hepatitis B. Liver Transpl 2013;19:887-895.

491. Fox AN, Terrault NA. The option of HBIG-free prophylaxis against recurrent HBV. J Hepatol 2012;56:1189-1197.

492. Wang P, Tam N, Wang H, Zheng H, Chen P, Wu L, et al. Is hepatitis $B$ immunoglobulin necessary in prophylaxis of hepatitis $B$ recurrence after liver transplantation? A meta-analysis. PLoS One 2014;9:e104480.

493. Fernández I, Loinaz C, Hernández O, Abradelo M, Manrique A, Calvo J, et al. Tenofovir/entecavir monotherapy after hepatitis $B$ immunoglobulin withdrawal is safe and effective in the prevention of hepatitis B in liver transplant recipients. Transpl Infect Dis 2015;17:695-701.

494. Cholongitas E, Papatheodoridis GV, Burroughs AK. Liver grafts from anti-hepatitis $B$ core positive donors: a systematic review. J Hepatol 2010;52:272-279.

495. Wachs ME, Amend WJ, Ascher NL, Bretan PN, Emond J, Lake JR, et al. The risk of transmission of hepatitis B from $\operatorname{HBsAg}(-), \operatorname{HBcAb}(+)$, HBlgM(-) organ donors. Transplantation 1995;59:230-234.

496. Prieto M, Gómez MD, Berenguer M, Córdoba J, Rayón JM, Pastor M, et al. De novo hepatitis $B$ after liver transplantation from hepatitis $B$ core antibody-positive donors in an area with high prevalence of anti-HBC positivity in the donor population. Liver Transpl 2001;7:5158.

497. Chotiyaputta W, Pelletier SJ, Fontana RJ, Lok AS. Long-term efficacy of nucleoside monotherapy in preventing HBV infection in HBsAg-negative recipients of anti-HBc-positive donor livers. Hepatol Int 2010;4:707-715.

498. Avelino-Silva VI, D'Albuquerque LA, Bonazzi PR, Song AT, Miraglia $J L$, De Brito Neves A, et al. Liver transplant from Anti-HBc-positive, $\mathrm{HBsAg}$-negative donor into $\mathrm{HBsAg-negative} \mathrm{recipient:} \mathrm{is} \mathrm{it} \mathrm{safe?} \mathrm{A}$ systematic review of the literature. Clin Transplant 2010;24:735746.

499. Saab S, Waterman B, Chi AC, Tong MJ. Comparison of different immunoprophylaxis regimens after liver transplantation with hepatitis B core antibody-positive donors: a systematic review. Liver Transpl 2010;16:300-307.

500. Lee KW, Lee DS, Lee HH, Kim SJ, Joh JW, Seo JM, et al. Prevention of de novo hepatitis $B$ infection from HbcAb-positive donors in living donor liver transplantation. Transplant Proc 2004;36:23112312.

501. Takemura N, Sugawara Y, Tamura S, Makuuchi M. Liver transplantation using hepatitis $B$ core antibody-positive grafts: review and university of Tokyo experience. Dig Dis Sci 2007;52:2472-2477.

502. Wright AJ, Fishman JA, Chung RT. Lamivudine compared with newer antivirals for prophylaxis of hepatitis $B$ core antibody positive livers: a cost-effectiveness analysis. Am J Transplant 2014;14:629634.

503. Fontana RJ, Hann HW, Wright T, Everson G, Baker A, Schiff ER, et al. A multicenter study of lamivudine treatment in 33 patients with hepatitis B after liver transplantation. Liver Transpl 2001;7:504510.

504. Ben-Ari Z, Mor E, Shapira Z, Tur-Kaspa R. Long-term experience with lamivudine therapy for hepatitis B virus infection after liver 
transplantation. Liver Transpl 2001;7:113-117.

505. Perrillo R, Rakela J, Dienstag J, Levy G, Martin P, Wright T, et al. Multicenter study of lamivudine therapy for hepatitis $B$ after liver transplantation. Lamivudine Transplant Group. Hepatology 1999;29:1581-1586.

506. Ben-Ari Z, Pappo 0, Zemel R, Mor E, Tur-Kaspa R. Association of lamivudine resistance in recurrent hepatitis $B$ after liver transplantation with advanced hepatic fibrosis. Transplantation 1999;68:232-236.

507. Neff GW, O'Brien CB, Nery J, Shire N, Montalbano M, Ruiz P, et al. Outcomes in liver transplant recipients with hepatitis $B$ virus: resistance and recurrence patterns from a large transplant center over the last decade. Liver Transpl 2004;10:1372-1378.

508. Fornairon S, Pol S, Legendre C, Carnot F, Mamzer-Bruneel MF, Brechot $C$, et al. The long-term virologic and pathologic impact of renal transplantation on chronic hepatitis B virus infection. Transplantation 1996;62:297-299.

509. Ahn HJ, Kim MS, Kim YS, Kim SI, Huh KH, Ju MK, et al. Clinical outcome of renal transplantation in patients with positive pretransplant hepatitis B surface antigen. J Med Virol 2007;79:16551663.

510. Cosconea S, Fontaine $H$, Méritet JF, Corouge $M$, Sogni $P$, ValletPichard $A$, et al. Benefits associated with antiviral treatment in kidney allograft recipients with chronic hepatitis B virus infection. J Hepatol 2012;57:55-60.

511. Yap DY, Tang CS, Yung S, Choy BY, Yuen MF, Chan TM. Longterm outcome of renal transplant recipients with chronic hepatitis B infection-impact of antiviral treatments. Transplantation 2010;90:325-330.

512. Yap DY, Yung S, Tang CS, Seto WK, Ma MK, Mok MM, et al. Entecavir treatment in kidney transplant recipients infected with hepatitis B. Clin Transplant 2014;28:1010-1015.

513. Lee J, Cho JH, Lee JS, Ahn DW, Kim CD, Ahn C, et al. Pretransplant hepatitis $B$ viral infection increases risk of death after kidney transplantation: a multicenter cohort study in Korea. Medicine (Baltimore) 2016;95:e3671.

514. Shin HS, Cho HJ, Jeon ES, Hwang HY, Kim JJ, Kim KB, et al. The impact of hepatitis $B$ on heart transplantation: 19 years of national experience in Korea. Ann Transplant 2014;19:182-187.

515. Durante-Mangoni E, Vitrone M, Parrella A, Andini R, lossa D, Ragone $E$, et al. Efficacy and safety of tenofovir, entecavir, and telbivudine for chronic hepatitis B in heart transplant recipients. Transpl Infect Dis 2016;18:319-325.

516. Jeon JW, Kim SM, Cho H, Baek CH, Kim H, Shin S, et al. Presence of hepatitis $B$ surface antibody in addition to hepatitis $B$ core antibody confers protection against hepatitis $B$ virus infection in hepatitis B surface antigen-negative patients undergoing kidney transplantation. Transplantation 2018;102:1717-1723.
517. Lee J, Park JY, Huh KH, Kim BS, Kim MS, Kim SI, et al. Rituximab and hepatitis $\mathrm{B}$ reactivation in $\mathrm{HBsAg}$-negative/ anti-HBc-positive kidney transplant recipients. Nephrol Dial Transplant 2017;32:722729.

518. Masutani K, Omoto K, Okumi M, Okabe Y, Shimizu T, Tsuruya K, et al. Incidence of hepatitis $B$ viral reactivation after kidney transplantation with low-dose rituximab administration. Transplantation 2018;102:140-145.

519. Mahboobi N, Tabatabaei SV, Blum HE, Alavian SM. Renal grafts from anti-hepatitis $B$ core-positive donors: a quantitative review of the literature. Transpl Infect Dis 2012;14:445-451.

520. Chamorro C, Aparicio M. Influence of anti-HBc positive organ donor in lung donor selection. Arch Bronconeumol 2012;48:320-324.

521. Huprikar S, Danziger-Isakov L, Ahn J, Naugler S, Blumberg E, Avery RK, et al. Solid organ transplantation from hepatitis B virus-positive donors: consensus guidelines for recipient management. Am J Transplant 2015;15:1162-1172.

522. Lau GK, Leung YH, Fong DY, Au WY, Kwong YL, Lie A, et al. High hepatitis $B$ virus (HBV) DNA viral load as the most important risk factor for HBV reactivation in patients positive for HBV surface antigen undergoing autologous hematopoietic cell transplantation. Blood 2002;99:2324-2330.

523. Li J, Liu J, Huang B, Zheng D, Chen M, Zhou Z, et al. Hepatitis B virus infection status is an independent risk factor for multiple myeloma patients after autologous hematopoietic stem cell transplantation. Tumour Biol 2013;34:1723-1728.

524. Shang J, Wang H, Sun J, Fan Z, Huang F, Zhang Y, et al. A comparison of lamivudine vs entecavir for prophylaxis of hepatitis B virus reactivation in allogeneic hematopoietic stem cell transplantation recipients: a single-institutional experience. Bone Marrow Transplant 2016;51:581-586.

525. Siyahian A, Malik SU, Mushtaq A, Howe CL, Majeed A, Zangeneh T, et al. Prophylaxis for hepatitis $B$ virus reactivation after allogeneic stem cell transplantation in the era of drug resistance and newer antivirals: a systematic review and meta-analysis. Biol Blood Marrow Transplant 2018;24:1483-1489.

526. Seto WK, Chan TS, Hwang YY, Wong DK, Fung J, Liu KS, et al. Hepatitis $B$ reactivation in occult viral carriers undergoing hematopoietic stem cell transplantation: a prospective study. Hepatology 2017;65:1451-1461.

527. Kim YJ, Lee JW, Kim YS, Jeong SH, Kim YS, Yim HJ, et al. Clinical features and treatment efficacy of peginterferon alfa plus ribavirin in chronic hepatitis C patients coinfected with hepatitis B virus. Korean J Hepatol 2011;17:199-205.

528. Choi MS, Sinn DH, Kim SA, Lee YS, Choi W, Paik SW. The clinical and laboratory characteristics of patients with chronic hepatitis B using current or past antiviral therapy in Korea: a multi-center, nation-wide, cross-sectional epidemiologic study. Gut Liver 
2012;6:241-248.

529. Lee LP, Dai CY, Chuang WL, Chang WY, Hou NJ, Hsieh MY, et al. Comparison of liver histopathology between chronic hepatitis $C$ patients and chronic hepatitis B and C-coinfected patients. J Gastroenterol Hepatol 2007;22:515-517.

530. Pol S, Haour G, Fontaine H, Dorival C, Petrov-Sanchez V, Bourliere $M$, et al. The negative impact of HBV/HCV coinfection on cirrhosis and its consequences. Aliment Pharmacol Ther 2017;46:10541060.

531. Chen G, Wang C, Chen J, Ji D, Wang Y, Wu V, et al. Hepatitis B reactivation in hepatitis $B$ and $C$ coinfected patients treated with antiviral agents: a systematic review and meta-analysis. Hepatology 2017;66:13-26.

532. Liu CJ, Chuang WL, Sheen IS, Wang HY, Chen CY, Tseng KC, et al. Efficacy of ledipasvir and sofosbuvir treatment of HCV infection in patients coinfected with HBV. Gastroenterology 2018;154:989997.

533. Bersoff-Matcha SJ, Cao K, Jason M, Ajao A, Jones SC, Meyer T, et al. Hepatitis $B$ virus reactivation associated with direct-acting antiviral therapy for chronic hepatitis $C$ virus: a review of cases reported to the U.S. Food and Drug Administration adverse event reporting system. Ann Intern Med 2017;166:792-798.

534. Solas C, Bregigeon S, Faucher-Zaegel O, Quaranta S, Obry-Roguet $\mathrm{V}$, Tamalet $\mathrm{C}$, et al. Ledipasvir and tenofovir drug interaction in human immunodeficiency virus-hepatitis $C$ virus coinfected patients: impact on tenofovir trough concentrations and renal safety. $\mathrm{Br}$ J Clin Pharmacol 2018;84:404-409.

535. Thio CL, Seaberg EC, Skolasky R Jr, Phair J, Visscher B, Muñoz A, et al. HIV-1, hepatitis $B$ virus, and risk of liver-related mortality in the Multicenter Cohort Study (MACS). Lancet 2002;360:1921-1926.

536. Kim YC, Ahn JY, Kim JM, Kim YJ, Park DW, Yoon YK, et al. Human immunodeficiency virus (HIV) and hepatitis virus coinfection among HIV-infected Korean patients: the Korea HIVIAIDS cohort study. Infect Chemother 2017;49:268-274.

537. World Health Organization (WHO). Consolidated guidelines on the use of antiretroviral drugs for treating and preventing HIV infection: recommendations for a public health approach. 2nd ed. Geneva: WHO, 2016:208.

538. Wranke A, Pinheiro Borzacov LM, Parana R, Lobato C, Hamid S, Ceausu $E$, et al. Clinical and virological heterogeneity of hepatitis delta in different regions world-wide: the Hepatitis Delta International Network (HDIN). Liver Int 2018;38:842-850.

539. Jeong SH, Kim JM, Ahn HJ, Park MJ, Paik KH, Choi W, et al. The prevalence and clinical characteristics of hepatitis-delta infection in Korea. Korean J Hepatol 2005;11:43-50.

540. Kim HS, Kim SJ, Park HW, Shin WG, Kim KH, Lee JH, et al. Prevalence and clinical significance of hepatitis $D$ virus coinfection in patients with chronic hepatitis B in Korea. J Med Virol
2011;83:1172-1177.

541. Bahcecioglu IH, Aygun C, Gozel N, Poyrazoglu OK, Bulut Y, Yalniz M. Prevalence of hepatitis delta virus (HDV) infection in chronic hepatitis B patients in eastern Turkey: still a serious problem to consider. J Viral Hepat 2011;18:518-524.

542. Fattovich G, Giustina G, Christensen E, Pantalena M, Zagni I, Realdi $G$, et al. Influence of hepatitis delta virus infection on morbidity and mortality in compensated cirrhosis type B. The European Concerted Action on Viral Hepatitis (Eurohep). Gut 2000;46:420426.

543. Triantos C, Kalafateli M, Nikolopoulou V, Burroughs A. Metaanalysis: antiviral treatment for hepatitis D. Aliment Pharmacol Ther 2012;35:663-673.

544. Wedemeyer H, Yurdaydin C, Dalekos GN, Erhardt A, Çakaloğlu Y, Değertekin $\mathrm{H}$, et al. Peginterferon plus adefovir versus either drug alone for hepatitis delta. N Engl J Med 2011;364:322-331.

545. Abbas Z, Memon MS, Mithani H, Jafri W, Hamid S. Treatment of chronic hepatitis $D$ patients with pegylated interferon: a real-world experience. Antivir Ther 2014;19:463-468.

546. Keskin O, Wedemeyer H, Tüzün A, Zachou K, Deda X, Dalekos GN, et al. Association between level of hepatitis $D$ virus RNA at week 24 of pegylated interferon therapy and outcome. Clin Gastroenterol Hepatol 2015;13:2342-2349.e1-e2.

547. Heidrich B, Yurdaydın C, Kabaçam G, Ratsch BA, Zachou K, Bremer $B$, et al. Late HDV RNA relapse after peginterferon alpha-based therapy of chronic hepatitis delta. Hepatology 2014;60:87-97.

548. Karaca C, Soyer OM, Baran B, Ormeci AC, Gokturk S, Aydin E, et al. Efficacy of pegylated interferon-a treatment for 24 months in chronic delta hepatitis and predictors of response. Antivir Ther 2013;18:561-566.

549. Söderström A, Norkrans G, Lindh M. Hepatitis B virus DNA during pregnancy and post partum: aspects on vertical transmission. Scand J Infect Dis 2003;35:814-819.

550. ter Borg MJ, Leemans WF, de Man RA, Janssen HL. Exacerbation of chronic hepatitis B infection after delivery. J Viral Hepat 2008;15:37-41.

551. Samadi Kochaksaraei G, Castillo E, Osman M, Simmonds K, Scott AN, Oshiomogho Jl, et al. Clinical course of 161 untreated and tenofovir-treated chronic hepatitis B pregnant patients in a low hepatitis B virus endemic region. J Viral Hepat 2016;23:15-22.

552. Chang CY, Aziz N, Poongkunran M, Javaid A, Trinh HN, Lau D, et al. Serum alanine aminotransferase and hepatitis B DNA flares in pregnant and postpartum women with chronic hepatitis B. Am J Gastroenterol 2016;111:1410-1415.

553. Fontana RJ. Side effects of long-term oral antiviral therapy for hepatitis B. Hepatology 2009;49(5 Suppl):S185-S195.

554. Zhou C, Yu Y, Yang Q, Wang H, Hou M, Jin L, et al. Motor development delay in offspring is associated with prenatal telbivudine 
exposure. Medicine (Baltimore) 2018;97:e0053.

555. Jacobson DL, Patel K, Williams PL, Geffner ME, Siberry GK, DiMeglio $L A$, et al. Growth at 2 years of age in HIV-exposed uninfected children in the United States by trimester of maternal antiretroviral initiation. Pediatr Infect Dis J 2017;36:189-197.

556. Nguyen G, Garcia RT, Nguyen N, Trinh H, Keeffe EB, Nguyen MH. Clinical course of hepatitis B virus infection during pregnancy. Aliment Pharmacol Ther 2009;29:755-764.

557. Chang CY, Aziz N, Poongkunran M, Javaid A, Trinh HN, Lau DT, et al. Serum aminotransferase flares in pregnant and postpartum women with current or prior treatment for chronic hepatitis B. J Clin Gastroenterol 2018;52:255-261.

558. Zhang L, Gui X, Fan J, Wang B, Ji H, Yisilafu R, et al. Breast feeding and immunoprophylaxis efficacy of mother-to-child transmission of hepatitis B virus. J Matern Fetal Neonatal Med 2014;27:182-186.

559. Benaboud S, Pruvost A, Coffie PA, Ekouévi DK, Urien S, Arrivé E, et al. Concentrations of tenofovir and emtricitabine in breast milk of HIV-1-infected women in Abidjan, Cote d'Ivoire, in the ANRS 12109 TEmAA Study, Step 2. Antimicrob Agents Chemother 2011;55:13151317.

560. Mirochnick M, Taha T, Kreitchmann R, Nielsen-Saines K, Kumwenda $N$, Joao $E$, et al. Pharmacokinetics and safety of tenofovir in HIV-infected women during labor and their infants during the first week of life. J Acquir Immune Defic Syndr 2014;65:33-41.

561. Waitt C, Olagunju A, Nakalema S, Kyohaire I, Owen A, Lamorde M, et al. Plasma and breast milk pharmacokinetics of emtricitabine, tenofovir and lamivudine using dried blood and breast milk spots in nursing African mother-infant pairs. J Antimicrob Chemother 2018;73:1013-1019.

562. World Health Organization (WHO). WHO Technical brief: Preventing HIV during pregnancy and breastfeeding in the context of preexposure prophylaxis (PrEP). Geneva: WHO, 2017:10.

563. Wen WH, Chang MH, Zhao LL, Ni YH, Hsu HY, Wu JF, et al. Motherto-infant transmission of hepatitis $B$ virus infection: significance of maternal viral load and strategies for intervention. J Hepatol 2013;59:24-30.

564. Zou H, Chen Y, Duan Z, Zhang H, Pan C. Virologic factors associated with failure to passive-active immunoprophylaxis in infants born to HBsAg-positive mothers. J Viral Hepat 2012;19:e18-e25.

565. Yu M, Jiang Q, Ji Y, Jiang $H$, Wu K, Ju $L$, et al. The efficacy and safety of antiviral therapy with lamivudine to stop the vertical transmission of hepatitis B virus. Eur J Clin Microbiol Infect Dis 2012;31:2211-2218.

566. Pan CQ, Han GR, Jiang HX, Zhao W, Cao MK, Wang CM, et al. Telbivudine prevents vertical transmission from $\mathrm{HBeAg}$-positive women with chronic hepatitis B. Clin Gastroenterol Hepatol 2012;10:520-526.

567. Chen HL, Lee CN, Chang CH, Ni YH, Shyu MK, Chen SM, et al.
Efficacy of maternal tenofovir disoproxil fumarate in interrupting mother-to-infant transmission of hepatitis $B$ virus. Hepatology 2015;62:375-386

568. Pan CQ, Duan Z, Dai E, Zhang S, Han G, Wang Y, et al. Tenofovir to prevent hepatitis $B$ transmission in mothers with high viral load. N Engl J Med 2016;374:2324-2334.

569. Hyun MH, Lee YS, Kim JH, Je JH, Yoo YJ, Yeon JE, et al. Systematic review with meta-analysis: the efficacy and safety of tenofovir to prevent mother-to-child transmission of hepatitis B virus. Aliment Pharmacol Ther 2017;45:1493-1505.

570. Jourdain G, Ngo-Giang-Huong N, Harrison L, Decker L, Khamduang $W$, Tierney $C$, et al. Tenofovir versus placebo to prevent perinatal transmission of hepatitis B. N Engl J Med 2018;378:911-923.

571. Kim JH, Lee YS, Lee HS, Chang SW, Yeon JE, Byun KS. The role of tenofovir for prevention of vertical transmission of hepatitis $B$ virus: systematic review with meta-analysis [Abstract]. Gut Liver 2018;12(Suppl 1):S109.

572. Hong SJ, Park HJ, Chu MA, Choi BS, Choe BH. The rate of conversion from immune-tolerant phase to early immune-clearance phase in children with chronic hepatitis B virus infection. Pediatr Gastroenterol Hepatol Nutr 2014;17:41-46.

573. Wu JF, Su YR, Chen $\mathrm{CH}$, Chen HL, Ni YH, Hsu HY, et al. Predictive effect of serial serum alanine aminotransferase levels on spontaneous $\mathrm{HBeAg}$ seroconversion in chronic genotype B and C HBVinfected children. J Pediatr Gastroenterol Nutr 2012;54:97-100.

574. Zhu S, Zhang H, Dong Y, Wang L, Xu Z, Liu W, et al. Antiviral therapy in hepatitis $B$ virus-infected children with immune-tolerant characteristics: a pilot open-label randomized study. J Hepatol 2018;68:1123-1128.

575. Jonas MM, Block JM, Haber BA, Karpen SJ, London WT, Murray KF, et al. Treatment of children with chronic hepatitis B virus infection in the United States: patient selection and therapeutic options. Hepatology 2010;52:2192-2205.

576. Sokal EM, Conjeevaram HS, Roberts EA, Alvarez F, Bern EM, Goyens $P$, et al. Interferon alfa therapy for chronic hepatitis $B$ in children: a multinational randomized controlled trial. Gastroenterology 1998;114:988-995.

577. Kobak GE, MacKenzie T, Sokol RJ, Narkewicz MR. Interferon treatment for chronic hepatitis B: enhanced response in children 5 years old or younger. J Pediatr 2004;145:340-345.

578. Wirth S, Zhang H, Hardikar W, Schwarz KB, Sokal E, Yang W, et al. Efficacy and safety of peginterferon alfa-2a (40KD) in children with chronic hepatitis B: the PEG-B-ACTIVE study. Hepatology 2018;68:1681-1694.

579. Jonas MM, Chang MH, Sokal E, Schwarz KB, Kelly D, Kim KM, et al. Randomized, controlled trial of entecavir versus placebo in children with hepatitis B envelope antigen-positive chronic hepatitis B. Hepatology 2016;63:377-387. 
580. Lee KJ, Choe BH, Choe JY, Kim JY, Jeong IS, Kim JW, et al. A multicenter study of the antiviral efficacy of entecavir monotherapy compared to lamivudine monotherapy in children with nucleos $(\mathrm{t})$ ide-naïve chronic hepatitis B. J Korean Med Sci 2018;33:e63.

581. Murray KF, Szenborn L, Wysocki J, Rossi S, Corsa AC, Dinh P, et al. Randomized, placebo-controlled trial of tenofovir disoproxil fumarate in adolescents with chronic hepatitis B. Hepatology 2012;56:2018-2026.

582. Choe JY, Ko JS, Choe BH, Kim JE, Kang B, Lee KJ, et al. Antiviral efficacy of tenofovir monotherapy in children with nucleos(t)idenaive chronic hepatitis B. J Korean Med Sci 2018;33:e11.

583. Jonas MM, Mizerski J, Badia IB, Areias JA, Schwarz KB, Little NR, et al. Clinical trial of lamivudine in children with chronic hepatitis $B$. N Engl J Med 2002;346:1706-1713.
584. Sokal EM, Kelly DA, Mizerski J, Badia IB, Areias JA, Schwarz KB, et al. Long-term lamivudine therapy for children with $\mathrm{HBeAg}$-positive chronic hepatitis B. Hepatology 2006;43:225-232.

585. Choe BH, Lee JH, Jang YC, Jang CH, Oh KW, Kwon S, et al. Longterm therapeutic efficacy of lamivudine compared with interferonalpha in children with chronic hepatitis B: the younger the better. J Pediatr Gastroenterol Nutr 2007:44:92-98.

586. Koh H, Baek SY, Chung KS. Lamivudine therapy for Korean children with chronic hepatitis B. Yonsei Med J 2007;48:927-933.

587. Chu M, Cho SM, Choe BH, Cho MH, Kwon S, Lee WK. Virologic responses to add-on adefovir dipivoxil treatment versus entecavir monotherapy in children with lamivudine-resistant chronic hepatitis B. J Pediatr Gastroenterol Nutr 2012;55:648-652. 